Copyright

by

Xun Li

2018 
The Dissertation Committee for Xun Li certifies that this is the approved version of the following Dissertation:

\title{
OPPONENT MODELING AND EXPLOITATION IN POKER USING EVOLVED RECURRENT NEURAL NETWORKS
}

\author{
Committee:
}

Risto Miikkulainen, Supervisor

Dana Ballard

Benito Fernandez

Aloysius Mok 


\title{
OPPONENT MODELING AND EXPLOITATION IN POKER USING EVOLVED RECURRENT NEURAL NETWORKS \\ by \\ Xun Li
}

\author{
Dissertation \\ Presented to the Faculty of the Graduate School of \\ The University of Texas at Austin \\ in Partial Fulfillment \\ of the Requirements \\ for the Degree of
}

Doctor of Philosophy

The University of Texas at Austin

August 2018 
To my mother, Rongsheng Li, my father, Weiru Guo, and my love, Yuanyuan Zhou, for your support and patience. 


\section{Acknowledgements}

I would like to start by thanking my research supervisor, Dr. Risto Miikkulainen, for his invaluable guidance and support throughout my Ph.D. program. To me, he is not only a knowledgeable mentor but also a great friend.

I would also like to thank my committee, Dr. Dana Ballard, Dr. Benito Fernandez, and Dr. Aloysius Mok, for their suggestions and encouragement. Without their help, this dissertation would not have been possible.

In addition, I would like to extend my appreciation to my friends and colleagues in the Department of Computer Science at the University of Texas at Austin. It has been a great honor and pleasure working in this lively and inspiring community.

Last but not least, I would like to thank my beloved family. In particular, I would like to thank my Mom, who encouraged me to embark on my journey towards a Ph.D. It would not be possible for this dream to come true without her love and dedication.

This research was supported in part by NIH grant R01-GM105042, and NSF grants DBI-0939454 and IIS-0915038.

The University of Texas at Austin August 2018 


\title{
Abstract \\ Opponent Modeling and Exploitation in Poker Using Evolved Recurrent Neural Networks
}

\author{
Xun Li, Ph.D. \\ The University of Texas at Austin, 2018
}

Supervisor: Risto Miikkulainen

As a classic example of imperfect information games, poker, in particular, HeadsUp No-Limit Texas Holdem (HUNL), has been studied extensively in recent years. A number of computer poker agents have been built with increasingly higher quality. While agents based on approximated Nash equilibrium have been successful, they lack the ability to exploit their opponents effectively. In addition, the performance of equilibrium strategies cannot be guaranteed in games with more than two players and multiple Nash equilibria. This dissertation focuses on devising an evolutionary method to discover opponent models based on recurrent neural networks.

A series of computer poker agents called Adaptive System for Hold'Em (ASHE) were evolved for HUNL. ASHE models the opponent explicitly using Pattern Recognition Trees (PRTs) and LSTM estimators. The default and board-texture-based PRTs maintain statistical data on the opponent strategies at different game states. The Opponent Action Rate Estimator predicts the opponent's moves, and the Hand Range Estimator evaluates 
the showdown value of ASHE's hand. Recursive Utility Estimation is used to evaluate the expected utility/reward for each available action.

Experimental results show that (1) ASHE exploits opponents with high to moderate level of exploitability more effectively than Nash-equilibrium-based agents, and (2) ASHE can defeat top-ranking equilibrium-based poker agents. Thus, the dissertation introduces an effective new method to building high-performance computer agents for poker and other imperfect information games. It also provides a promising direction for future research in imperfect information games beyond the equilibrium-based approach. 


\section{Table of Contents}

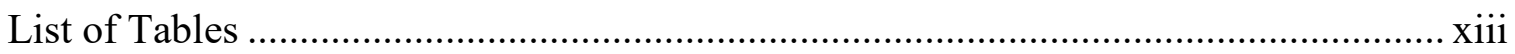

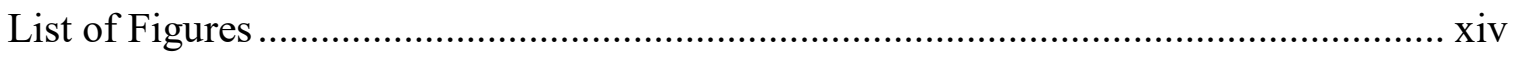

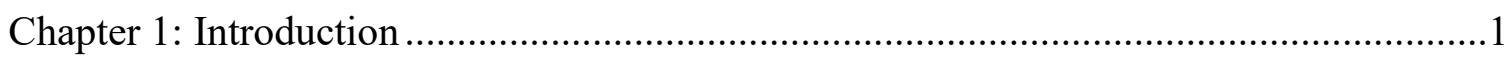

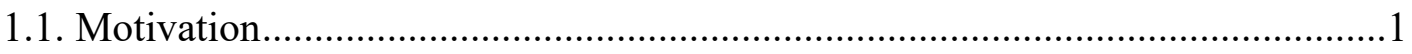

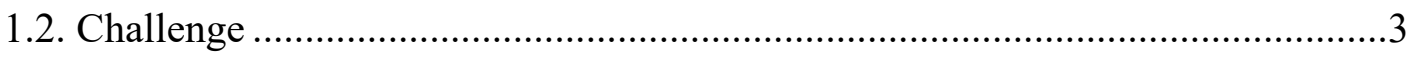

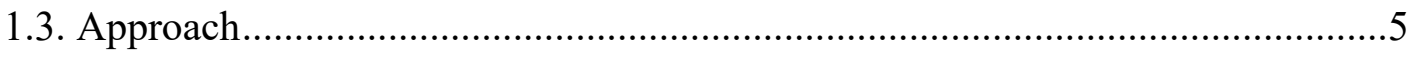

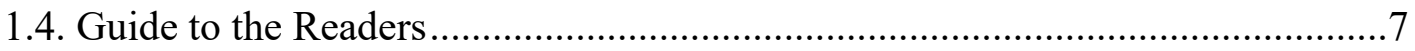

Chapter 2: Heads-Up No-Limit Texas Holdem .................................................................

2.1. Rules of the Game

2.2. Rankings of Hands in Texas Holdem ............................................................12

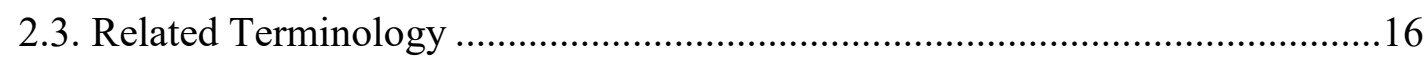

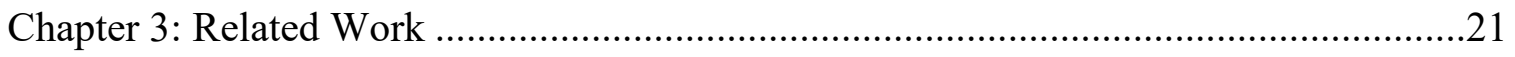

3.1. Computer Poker and Nash Equilibrium Approximation ...................................21

3.1.1. Simple Poker Variants ....................................................................22

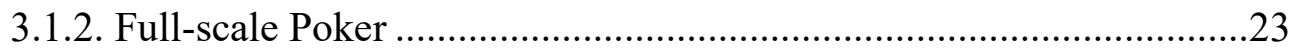

Equilibrium Approximation Techniques .............................................23

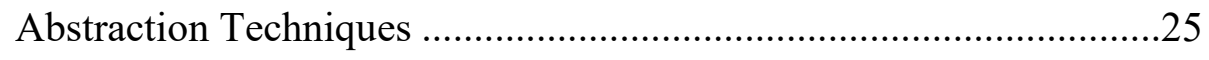

3.1.3. Conclusions on Nash Equilibrium Approximation..............................26

3.2. Opponent Modeling and Exploitation...............................................................2

3.2.1. Non-equilibrium-based Opponent Exploitation...................................27

Rule-based Statistical Models...........................................................28

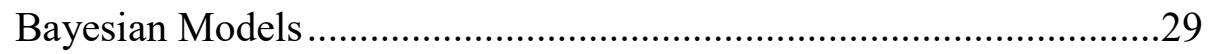


Neural Networks ............................................................................

Miscellaneous Techniques ..................................................................31

3.2.2. Equilibrium-based Opponent Exploitation ...........................................31

3.2.3. Conclusions on Opponent Modeling .......................................................34

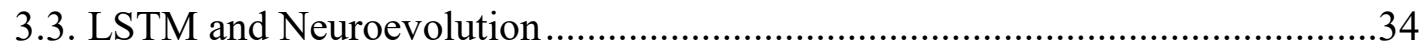

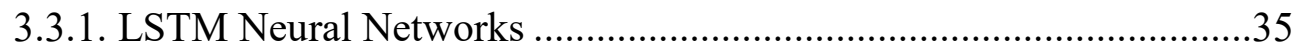

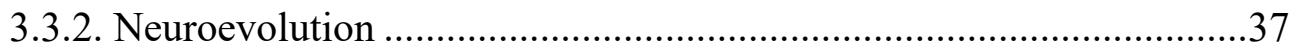

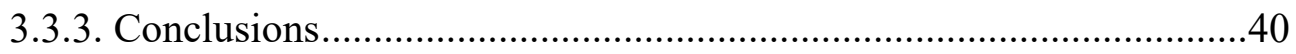

Chapter 4: Evolving LSTM-based Opponent Models ......................................................41

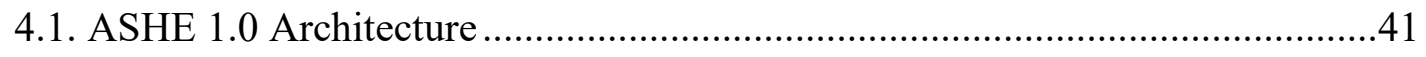

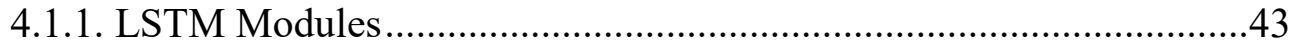

4.1.2. Decision Network and Decision Algorithm.........................................44

4.2. Method of Evolving Adaptive Agents ...............................................................45

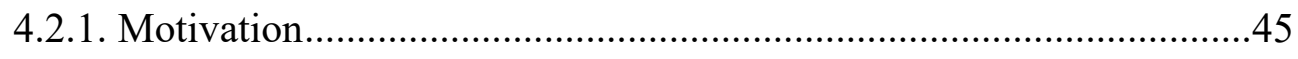

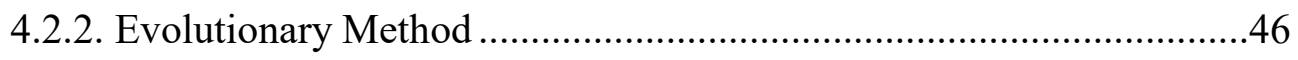

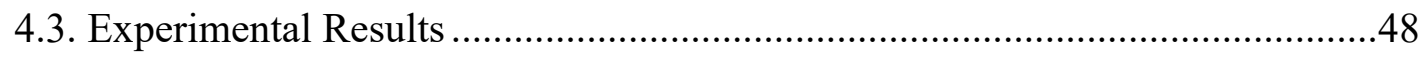

4.3.1. Experimental Setup......................................................................... 48

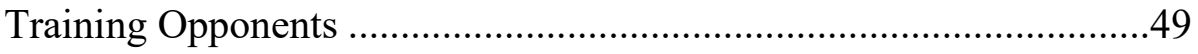

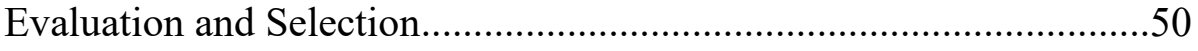

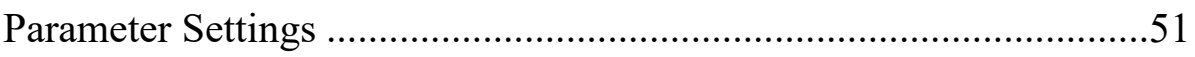

4.3.2. Adaptation, Exploitation, and Reliability ………..............................52

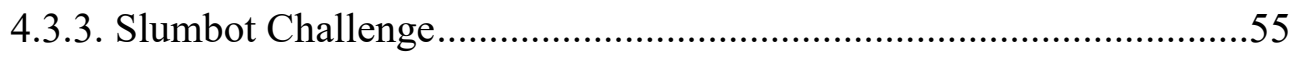

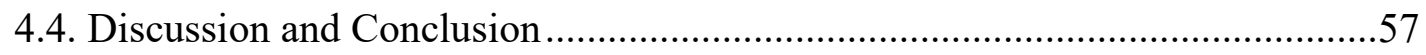




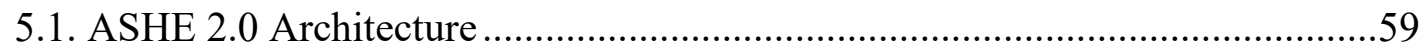

5.1.1. Motivation and Framework ……………………..............................6

Motivation: Pattern Recognition Tree ………………….......................60

Motivation: Explicit Modeling and Utility-based Decision-making ...62

5.1.2. Opponent Model ...............................................................................64

Pattern Recognition Tree ...................................................................64

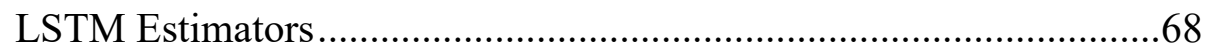

5.1.3. Decision Algorithm.........................................................................

5.2. Evolution of the LSTM Estimators...............................................................72

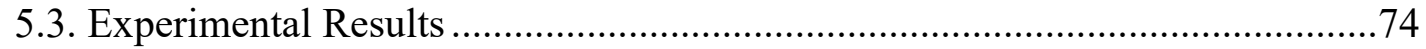

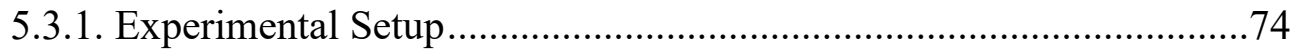

5.3.2. Training and Testing Opponents....................................................... 75

5.3.3. Results and Analysis .....................................................................

Performance vs. High-exploitability Opponents....................................78

Performance vs. Low-exploitability Opponents ...................................79

Performance vs. Dynamic Strategies ..................................................80

Training Opponent(s) and Performance...............................................8

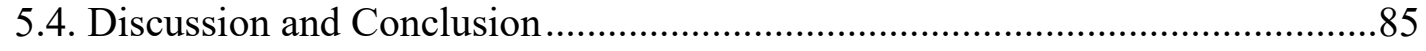

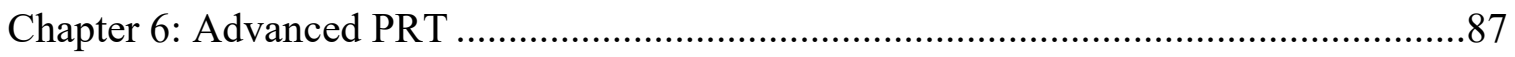

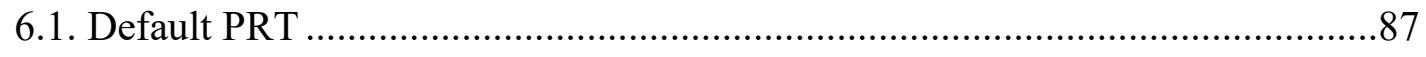

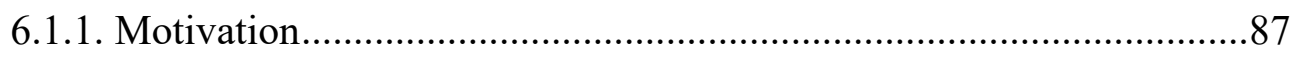

6.1.2. Construction and Application ...........................................................89 


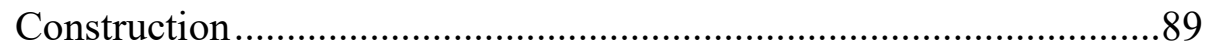

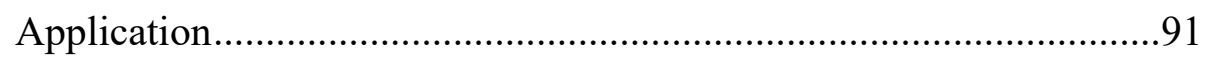

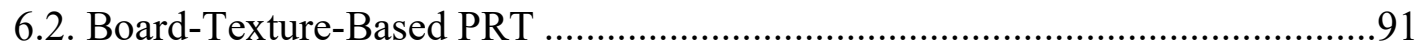

6.2.1. Motivation .......................................................................................... 92

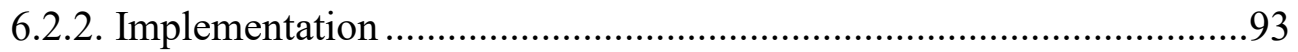

Board-texture-based PRT Structure ....................................................93

Board Texture Clusters ...................................................................94

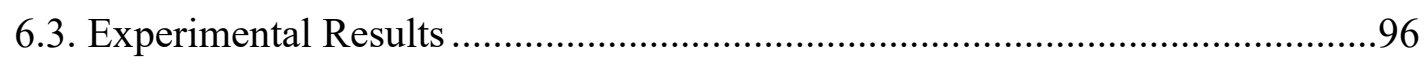

6.3.1. Experimental Setup .........................................................................96

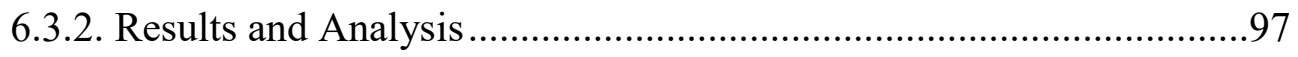

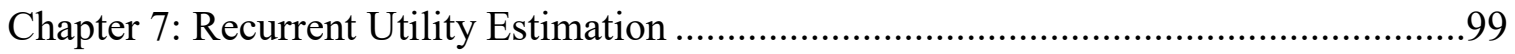

7.1. Recurrent Utility Estimation ..........................................................................99

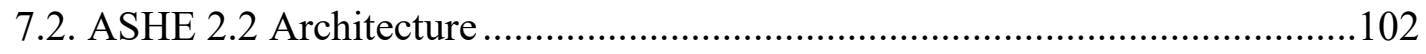

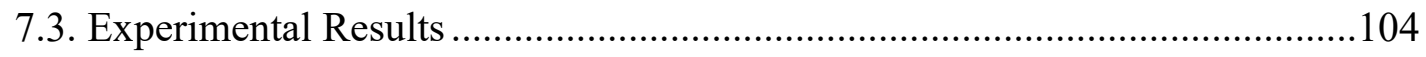

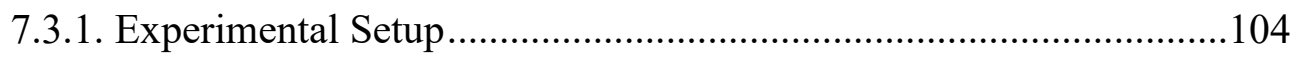

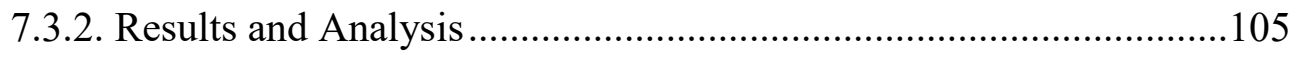

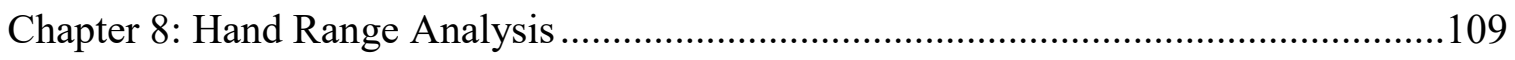

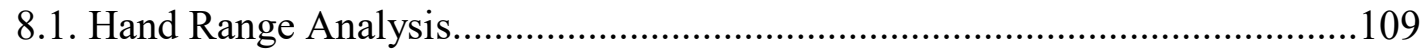

8.2. Hand Range Estimator and the ASHE 2.3 Architecture ..................................110

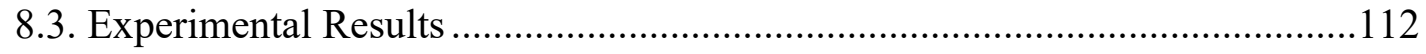

8.3.1. Evolving ASHE 2.3 ..................................................................113

8.3.2. Performance vs. Highly Exploitable Opponents.................................113

8.3.3. Tournament Evaluation...................................................................115 


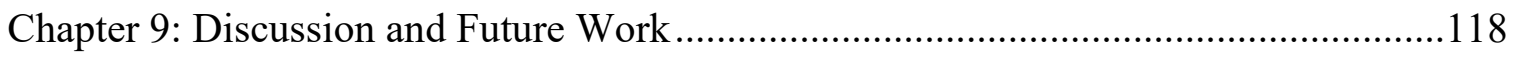

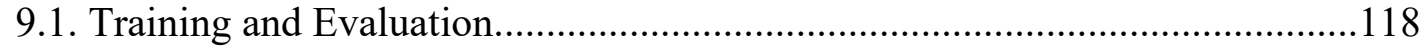

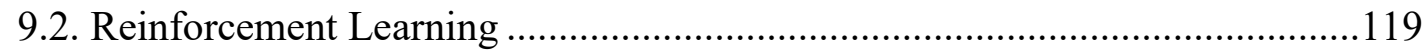

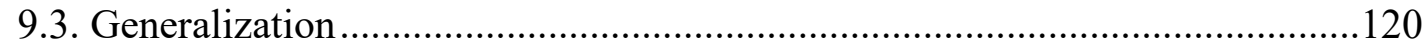

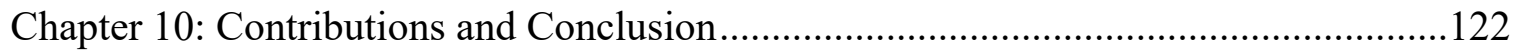

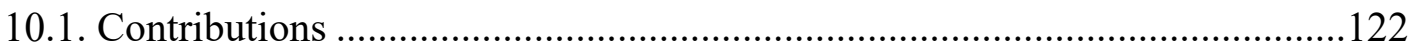

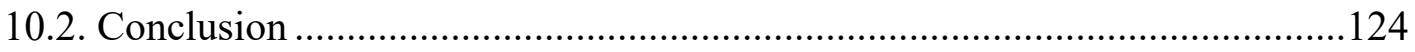

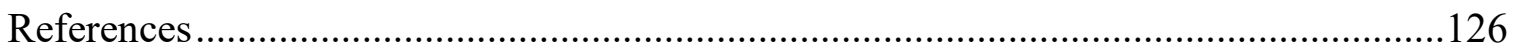




\section{List of Tables}

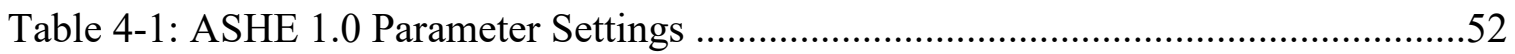

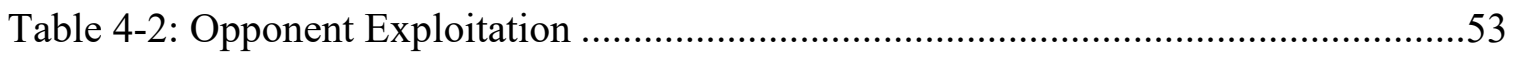

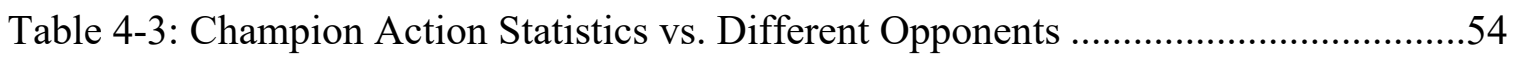

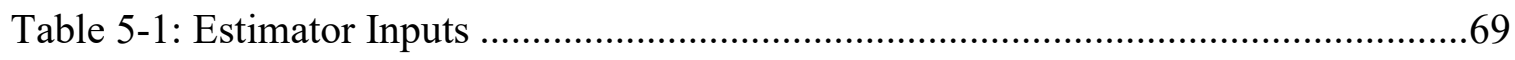

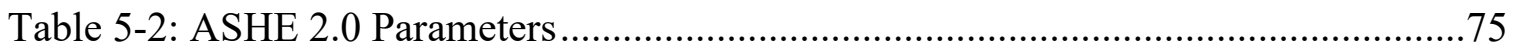

Table 5-3: Training and Testing Opponents ....................................................... 76

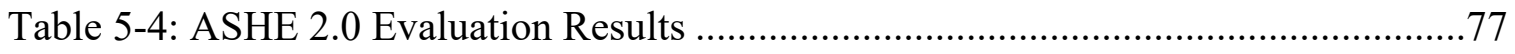

Table 5-5: ASHE 2.0 and ASHE 1.0 vs. Low-Exploitability Opponents ........................79

Table 5-6: Performance against Dynamic Strategies .................................................. 81

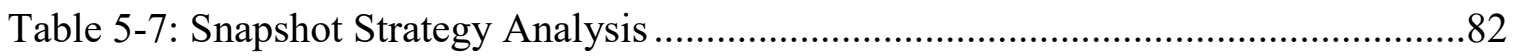

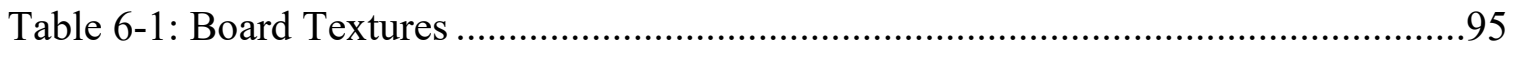

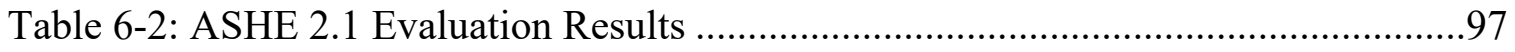

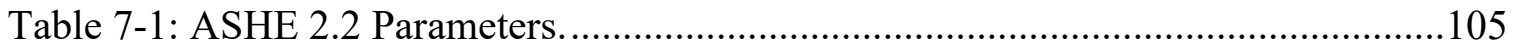

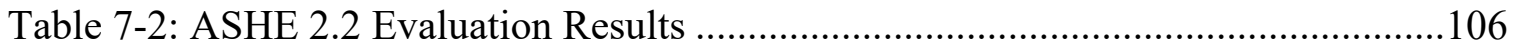

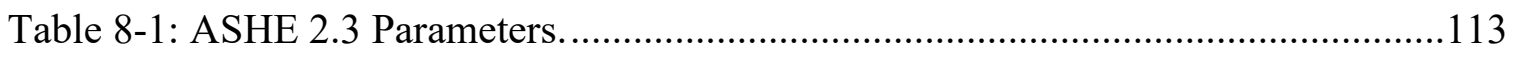

Table 8-2: ASHE Systems v. Highly Exploitable Opponents. .....................................114

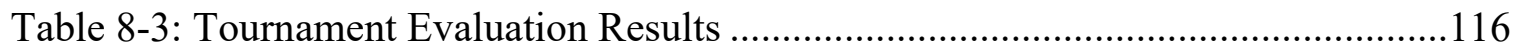




\section{List of Figures}

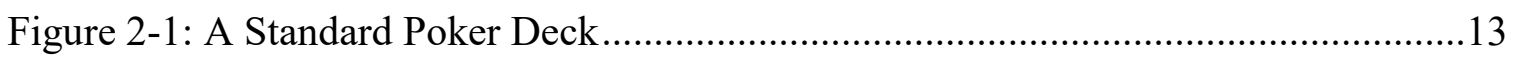

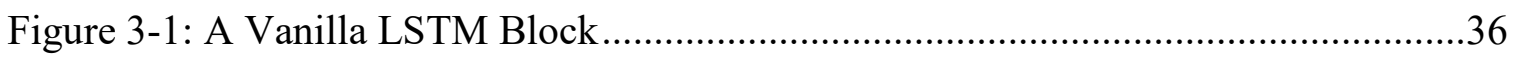

Figure 3-2: Genetic Algorithm Framework ....................................................................

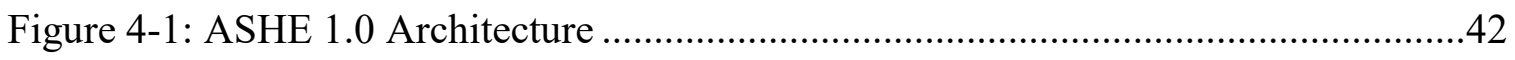

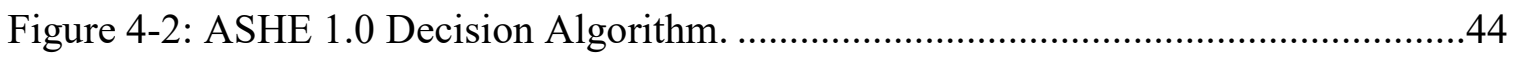

Figure 4-3: ASHE 1.0 Training Opponents ................................................................49

Figure 4-4: Champion Performance vs. Rule-based Players ………………………….....53

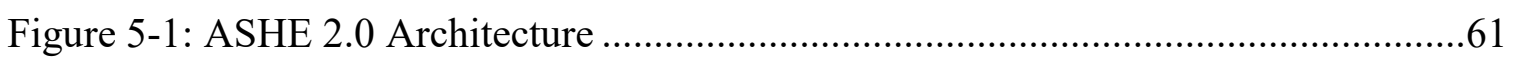

Figure 5-2: A Pattern Recognition Tree....................................................................65

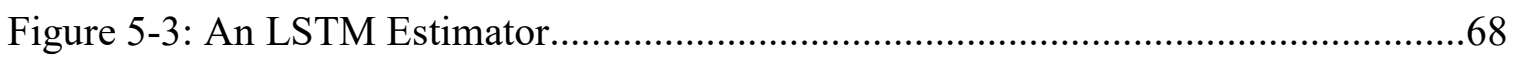

Figure 5-4: Decision Algorithm (ASHE 2.0) ................................................................71

Figure 6-1: ASHE with Default PRTs (ASHE 2.1) ......................................................90

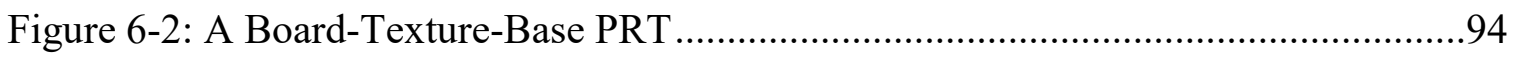

Figure 7-1: Recurrent Utility Estimation .....................................................................100

Figure 7-2: ASHE with Recursive Utility Estimation (ASHE 2.2) ………………….....102

Figure 7-3: Sample Game - ASHE 2.2 v. Slumbot 2017 ...............................................107

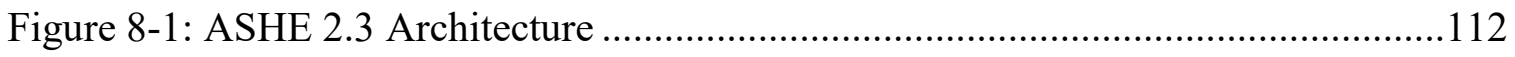




\section{Chapter 1: Introduction}

Imagine a world where computers are not only tools we use but also colleagues and collaborators with which we solve problems. For instance, you may be concerned about how to protect the safety of your community, or wish to have the upper hand in an important business negotiation, or simply hope to impress your friends in the next poker party. Many such challenges entail hidden secrets and cunning opponents, and it would be nice to have a loyal and intelligent computer partner on our side. Our tireless collaborators will help us identify weaknesses, hone our skills, and devise our strategies whenever and wherever we need. Such collaborators need to deal with imperfect information and deceptive opponents, and they must be able to exploit opponent strategies through adaptation. This dissertation is a step towards that goal.

\subsection{Motivation}

Imperfect information games are an important AI problem with numerous realworld applications, including cyber security, trading, business transaction and negotiation, military decision-making, table games, etc.

Computer agents in imperfect information games must address two challenges.

First, the agents are unable to fully observe the state of the game. Second, opponents may attempt to mislead the agents with deceptive actions. As an example, poker agents cannot observe their opponents' cards when making their moves (partial observation), and the opponents may slow-play a strong hand or bluff (deception).

Game theoretic analysis have been the most common approach to solve these problems. For instance, Sajjan et al. [2014] proposed a holistic cyber security approach in which the interaction between the attacks and the defense mechanisms was modeled as an 
imperfect information game. A game theory inspired defense architecture was developed to defend against dynamic adversary strategies.

Vatsa et al. [2005] modeled the conflicting motives between an attacker and a credit card fraud detection system as a multi-stage imperfect information game and proposed a fraud detection system based on a game-theoretic approach.

Business transaction and negotiation can be modeled as imperfect information games as well. For example, Cai and Wurman [2005] developed an agent that constructs a bidding policy in sealed-bid auction by sampling the valuation space of the opponents, solving corresponding complete information game, and aggregating the samples.

Wang and Wellman [2017] proposed an agent-based model of manipulating prices in the financial markets through spoofing. Empirical game-theoretic analysis showed that spoofing can be profitable in a market with heuristic belief learning traders. However, after re-equilibrating games with spoofing, such manipulation hurts market surplus and reduces the proportion of HBL traders.

As a classic imperfect information game, poker has been studied extensively in recent years. It is an ideal problem for the research on imperfect information games for the following reasons. First, most real world applications must be modeled as a formal game. This process is domain-specific and can be quite challenging. Moreover, a problem can be modeled in different ways, making it difficult to compare results. In contrast, poker games are already well-defined formal games. Hence, there is no need to model and formalize the problem. Experimental results are comparable for each variant of the game, and the techniques can be generalized to formal games derived from other problem domains.

Second, simple variants of poker is manually solvable, while complex variants such as No-limit Holdem can be extremely challenging. Therefore, poker games have been used as the target problem for both early and recent studies. 
Third, poker is one of the most popular games. Poker variants, e.g. Texas Holdem, Omaha, etc., are enjoyed by millions of players all over the world. Thus, research on poker is both fun and rewarding.

Existing work on poker focuses on two aspects: (1) equilibrium approximation and (2) opponent modeling. Equilibrium approximation techniques are designed to minimum exploitability, and opponent modeling techniques allow the agents to adapt to and exploit the opponents for higher utility.

This dissertation addresses opponent modeling problem from a new perspective. It applies genetic algorithms to evolve RNN-based adaptive agents for HUNL, providing an effective new approach to building high-performance computer agents for poker and other large-scale imperfect information games.

\subsection{Challenge}

Equilibrium-approximation techniques, e.g. Counterfactual Regret Minimization (CFR) have been applied to multiple variants of the game and have achieved remarkable successes [Zinkevinch et al., 2007]. In particular, Heads-Up Limit Texas Holdem has been weakly solved [Bowling et al., 2015]. Many powerful poker agents for Heads-Up No-Limit Holdem (HUNL) have been built through CFR in combination with various abstraction and/or sampling techniques [Gilpin and Sandholm, 2008a; Brown et al., 2015; Jackson, 2017]. In recent Human vs. AI competitions, Nash-equilibrium-based poker agents have defeated top professional human players in HUNL with statistically significant margins [Moravcik et al., 2017; Brown and Sandholm, 2017].

However, equilibrium-approximation approaches have three limitations. First, for imperfect information games with a large state space, the quality of Nash-equilibrium approximation can be far from ideal. While a real Nash-equilibrium strategy in a two- 
player zero-sum game such as HUNL is theoretically unexploitable (i.e. the game value of the best counter-strategy is zero), most top-ranking poker agents based on approximated Nash equilibrium strategies are exploitable with a simple local best response method [Lisy and Bowling, 2016].

Second, in imperfect information games with more than two players and multiple equilibria, if the opponents are not following the same equilibrium as approximated by an equilibrium-based agent, the agent's performance cannot be guaranteed [Ganzfried, 2016]. Therefore, it is difficult to apply equilibrium-approximation approaches to many imperfect information games with three or more players, such as poker tournaments, multi-party business negotiation, and security resource allocation.

Third, unexploitability does not guarantee maximum utility. In most real-world applications, the ultimate goal is not to become unexploitable but to achieve maximum utility against any opponent. In the case of poker, the goal is to win as many chips as possible. While a real equilibrium strategy is guaranteed not to lose money statistically to any opponent, it is unlikely to be the most profitable strategy.

The most effective counter-strategy against each opponent is different, and only through opponent modeling and adaptation, can a player approximate such counterstrategies for all opponents. Therefore, opponent modeling and adaptation may be the next step towards building stronger poker agents beyond Nash equilibrium approximation [ $\mathrm{Li}$ and Miikkulainen, 2017].

Existing work on opponent modeling lays a foundation for building high-quality adaptive poker agents. A number of computer agents capable of exploiting weak opponents have been built over the past two decades. However, few of them can achieve comparable performance playing against cutting-edge equilibrium-based agents in large-scale games such as HUNL (see Chapter 3 for details). 
In recent years, researchers attempted to build adaptive poker agents by adjusting precomputed equilibrium strategies according to opponent's action frequencies [Ganzfried and Sandholm, 2011 and 2015]. This approach reduces the exploitability of adaptive agents, thereby allowing them to perform better against other equilibrium-based opponents. Nevertheless, it only allows limited deviation from the Nash equilibrium strategy, thus reducing the effectiveness of opponent exploitation.

This dissertation aims at developing a method to build adaptive poker agents with recurrent-neural-network-based opponent models for HUNL. The goal of the research is to build agents that are effective in modeling a wide range of opponents and exploiting their weaknesses. In addition, they should achieve overall equivalent or better performance playing against cutting-edge equilibrium-based opponents. Such approach does not approximate equilibrium strategies and should be able to generalize to games with multiple players and equilibria.

\subsection{APPROACH}

This dissertation proposes an evolutionary method to discover opponent models based on recurrent neural networks. These models are combined with a decision-making algorithm to build agents that can exploit their opponents through adaptation.

In HUNL, opponent strategies are not known but must be learned from gameplay. While supervised learning is commonly adopted for training neural network models, the lack of sufficient labeled training data makes it rather difficult to apply such techniques for opponent modeling. In fact, even the best human players cannot agree on the probability for the opponent taking different actions given the current game state and the entire history of games played against that opponent. 
A particular powerful approach for such domains is evolutionary computation: in many comparisons with reinforcement learning, it has achieved better performance in discovering effective strategies from gameplay [Stanley et al., 2005; Li and Miikkulainen, 2018]. Evolution evaluates the agents based on their fitness, i.e. the overall performance against different opponents rather than specific predictions, thus requiring no labeled training data.

To evaluate the proposed approach, a series of poker agents called Adaptive System for Hold'Em (ASHE) were evolved for HUNL. ASHE models the opponent using Pattern Recognition Trees (PRTs) and LSTM estimators.

The PRTs store and maintain statistical data on the opponent's strategy. They are introduced to capture exploitable patterns based on different game states, thus improving performance against strong opponents. They can also reduce the complexity of the LSTM modules, making evolution more efficient. There are two types of PRTs in ASHE: the default PRTs and the regular PRTs. The default PRTs are fixed and serve as a background model. They allow the agent to make effective moves when facing a new opponent or an infrequent game state. The regular PRTs are reset and updated for each opponent. As more games are played with the opponent, the regular PRTs data becomes increasingly reliable, allowing the agent to capture exploitable patterns in the opponent strategy.

The LSTM estimators are recurrent neural network modules, which are optimized through evolution. They receive sequential inputs derived from the game states and the corresponding PRT data. The Opponent Action Rate Estimator predicts the probability of different opponent actions. The Hand Range Estimator evaluates strength of the opponent's hand, allowing the agent to compute showdown value more accurately. 
Decisions are made via Recursive Utility Estimation. The utility of each available action is computed using the predictions from the LSTM estimators. Action utilities are evaluated by aggregating possible future moves and corresponding results.

The above techniques are introduced separately in ASHE 1.0 through ASHE 2.3, each technique improves ASHE's performance substantially, leading to a system that can defeat top-ranking Nash-equilibrium-based agents and outperform them significantly when playing against weaker opponents.

\subsection{GUIDE TO THE READERS}

This dissertation is organized as follows:

Chapter 2 introduces the problem domain, i.e. Heads-Up No Limit Texas Holdem. It specifies the rules of the game and rankings of the hands. It also defines common poker terminology that is used in this dissertation.

Chapter 3 outlines related work on computer poker, including the state-of-the-art approach of Nash Equilibrium Approximation, and existing work on opponent modeling and exploitation in poker. It also presents the technical foundation of ASHE, including recurrent neural networks and neuroevolution.

Chapter 4 introduces ASHE 1.0, a two-module LSTM-based agent evolved by playing against highly exploitable opponents. ASHE 1.0 validates the methodology and establishes a framework for evolving adaptive poker agents.

Chapter 5 presents ASHE 2.0, which employs Pattern Recognition Trees (PRTs) to models the opponent explicitly. Experimental results in this chapter show that the system is significantly more effective in exploiting weak opponents than equilibrium-based agents and that it achieves comparable performance in matches against top-ranking poker agents. 
Chapter 6 introduces ASHE 2.1 and advanced PRTs, which is capable of modeling and exploiting patterns in opponent strategies that are related to board texture.

Chapter 7 introduces ASHE 2.2 and Recursive Utility Estimation. This technique provides more accurate evaluation of action utilities, thus allowing the adaptive agent to apply advanced poker tactics effectively.

Chapter 8 introduces ASHE 2.3 and Hand Range Estimator (HRE), which improves showdown value estimation through range analysis. ASHE 2.3 outperforms top-ranking equilibrium-based agents by a significant margin and is highly effective against opponents with different level of exploitability.

Chapter 9 discusses the experimental results and points out potential directions for future work, including using stronger training opponents, reinforcement learning, and generalization to other imperfect information games.

Chapter 10 reviews the contributions of this dissertation and summarizes the conclusions from it. 


\section{Chapter 2: Heads-Up No-Limit Texas Holdem}

Texas Holdem is a popular variation of the card game of poker. No-Limit Texas Holdem is regarded as one of the most challenging problems among imperfect information games because of its enormous state space considering all possible combinations of hole cards, community cards, and action sequences.

As an example, Heads-Up No-Limit Holdem in the format adopted by the Annual Computer Poker Competition in recent years has approximately $6.31 \times 10^{164}$ game states and $6.37 \times 10^{161}$ observable states [Johanson, 2013]. On the other hand, unlike most real world applications, actions, states, and utilities (rewards) in Texas Holdem are clearly defined by the rules of the game, making it easy to model the game mathematically. Therefore, No-Limit Texas Holdem has become a classic problem in the research of imperfect information games.

This dissertation, like most current researches on No-Limit Texas Holdem, focuses on the two-player version of the game, i.e. Heads-Up No-Limit Holdem (HUNL). The rest of this chapter presents the rules of the game and defines most poker terminology used in this dissertation.

\subsection{RULES OF THE GAME}

In HUNL, two players compete for money or chips contributed by both of them, i.e. the pot. At the beginning of each game, both players are forced to post a bet into the pot, i.e. the blinds. One of the players posts a smaller bet called the Small Blind, the other posts a bigger bet called the Big Blind. Usually, the big blind is twice the small blind, and a "\$50/\$100 game" means a game with $\$ 50$ small blind and $\$ 100$ big blind.

Depending on the context, the term "Small Blind" and "Big Blind" may refer to the player posting the corresponding forced bet. In HUNL, the Small Blind (player) can be 
referred to as the "button" or the "dealer". In a game session with multiple games, the players play as the dealer and the Big Blind alternately.

At the beginning of a game, each player is dealt two cards, i.e. the hole cards, from a standard 52-card poker deck. The hole cards are private to the receiver, thus making the states of the game partially observable. The game is divided into four betting rounds: preflop, flop, turn, and river. The players acts alternately in each betting rounds. The dealer acts first preflop, and the big blind acts first in all other betting rounds. Players must choose one of the following actions when it is their turn to act:

Raise: To raise is to commit more chips than the opponent. A raise not only makes the pot bigger but also challenges the opponent to match the raise by calling, (re-)raising, or moving all-in.

Call: To call is to match the opponent's contribution to the pot. If the two players have already committed the same amount of money to the pot (i.e. the player does not need to commit more chips to match the opponent's contribution), the action is usually called "check".

All-in: To go all-in is to commit all chips into the pot. An all-in may or may not be a legitimate call or raise. In particular, if a player cannot afford to call after the opponent raises or moves all-in, the player may go allin in response.

Fold: To fold is to concede the pot to the opponent. It is the only way for a player to avoid committing more chips to the pot after a raise from the opponent.

By convention, the size of a raise refers to the difference between chips committed by the raiser after the raise and the chips committed by the opponent. If the opponent has 
not raised in the current betting round, this action is usually called a "bet" or an "open raise". The minimum size of an open raise is equal to the Big Blind. If the opponent raised in the current betting round, the minimum size of a raise is the size of the opponent's last raise.

If a player folds, the game ends, and the opponent wins the pot. Otherwise, the betting round continues until both players have taken at least one action and committed the same amount of chips into the pot.

As the game proceeds, five cards are dealt face up on the table. Each of them is a community card, and the set of community cards is called the board. Specifically, the board is empty preflop; three community cards are dealt at the beginning of the flop, a fourth community card is dealt at the beginning of the turn, and the last community card is dealt at the beginning of the river. The board is observable to both players throughout the game.

If neither player has folded by the end of the river, the game goes into a showdown. In addition, at any point of the game, if a player who has moved all-in contributes no more chips to the pot than the other, the game also goes into a showdown. In this case, unfinished betting rounds are skipped, and the board is completed immediately.

Note that if the players have contributed different amount of chips into the pot, the effective size of the pot is twice the amount committed by the player with less contribution. Any additional chips are returned to the player with more contribution.

In a showdown, each player combines the hole cards with the board and chooses five out of the seven cards (two hole cards plus five community cards) to form the best possible hand. The player with the better hand wins the pot. If the two hands are equally strong, the players split the pot. The next section presents the rankings of poker hands.

Thus, a player may win the pot in two ways: (1) forcing the opponent to fold before showdown, or (2) going into a showdown with a stronger hand. In principle, a player should 
choose actions based on their expected utilities in both aspects. The expected utilities of an action can be estimated based on a player's hole cards, the board, and the sequence of actions in the game. Furthermore, since an HUNL game session usually contains hundreds of games, the strategy of the opponent can be modeled and weaknesses exploited based on the history of previous games.

The huge number of possible game states and clear mathematical definition of HUNL make it one of the most challenging and interesting problems for research on building high-performance computer agents for large-scale imperfect information games.

\subsection{RaNkingS OF HaNdS in TEXas Holdem}

In a showdown, the winner is the player with a better five-card hand according to the rankings of hands. There are nine categories of hands in Texas Holdem. The categories are ranked based on the rarity of the hands. A hand in a higher-ranking category beats any hand in a lower-ranking category. Hands in the same category are ranked relative to each other by comparing the ranks of their respective cards.

Texas holdem uses standard 52-card poker deck, which contains four suits, i.e. Spades, Hearts, Diamonds, and Clubs. Each card has a rank. The ranks are: Ace (A), King $(\mathrm{K})$, Queen $(\mathrm{Q})$, Jack (J), $10(\mathrm{~T}), 9,8,7,6,5,4,3$, 2, with Ace being the highest and 2 the lowest. The suit of the cards do not affect their value in making a hand, e.g. Ace of Spades is the same as Ace of Clubs.

For convenience of reference, cards in the rest of this dissertation are represented by two-character strings where the first character represents the rank of the card, and the second character represents the suit of the card. For example, "Ks" refers to king of spades, "Th" Ten of Hearts, " $5 d$ " Five of Diamonds, and "2c" Two (a.k.a. Deuce) of Clubs. 


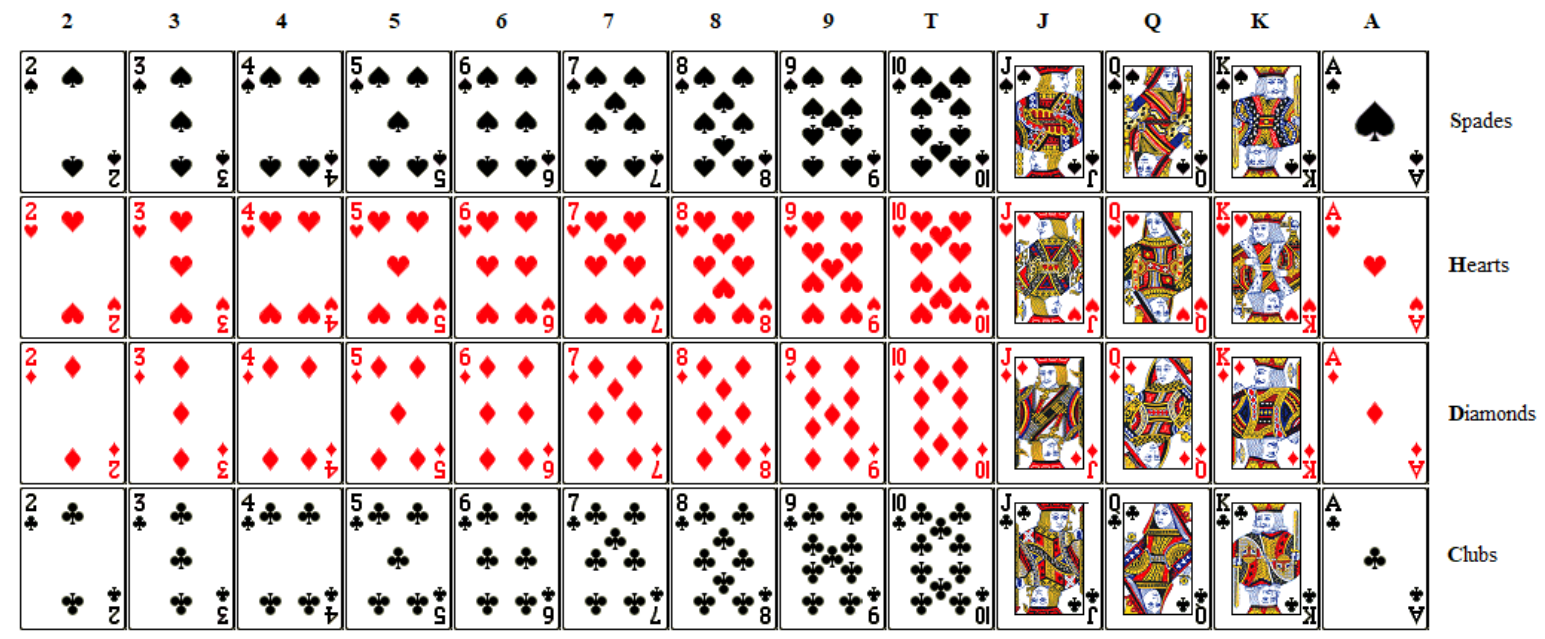

Figure 2-1: A Standard Poker Deck. Texas Holdem uses standard 52-card decks. Cards are split into four suits (Spades, Hearts, Diamonds, and Clubs) and thirteen ranks, with Ace being the highest and 2 the lowest.

The following list presents the nine categories of hands from the highest-ranking to the lowest-ranking.

Straight Flush: Straight flush is a hand containing five cards of sequential rank of the same suit. Hands within this category are ranked by the rank of their highest-ranking card. Thus, Td9d8d7d6d ranks higher than $5 \mathrm{~d} 4 \mathrm{~d} 3 \mathrm{~d} 2 \mathrm{dAd}$, and lower than JdTd9d8d7d. The best straight flush, a.k.a. the royal flush, consists of ace to ten of a same suit, e.g. AsKsQsJsTs. In Texas Holdem, ace can be used as the lowest ranking card to form a five-high straight flush, e.g. 5h4h3h2hAh, which is the lowest-ranking straight flush. Note that straight flush requires at least three cards of the same suit from the five community cards; it is therefore impossible to have straight flush hands of different suits in a single game.

Four of a Kind: Four of a kind, a.k.a. quads, is a hand containing four cards of the same rank and one card of another rank, e.g. TsThTdTc7c. Hands 
in this category are ranked first by the rank of their quadruplet, and then by the rank of the single card, i.e. the kicker. Thus, QsQhQdQcKh ranks the same as QsQhQdQcKc, higher than $2 \mathrm{~s} 2 \mathrm{~h} 2 \mathrm{~d} 2 \mathrm{cAs}$, and lower thanAsAhAdAc7d.

Full House: Full house, a.k.a. boat, is a hand containing three cards of the same rank and two cards (a pair) of another rank, e.g. KsKhKc2s2c. Hands within this category are ranked first by the rank of the triplet, and then by the rank of the pair. Therefore, $8 \mathrm{~s} 8 \mathrm{~h} 8 \mathrm{~d} 3 \mathrm{~h} 3 \mathrm{c}$ ranks the same as $8 \mathrm{~s} 8 \mathrm{~h} 8 \mathrm{c} 3 \mathrm{~h} 3 \mathrm{~d}$, higher than $3 \mathrm{~s} 3 \mathrm{~h} 3 \mathrm{c} 8 \mathrm{~s} 8 \mathrm{~h}$, and lower than AsAhAc2s2c.

Flush: Flush is a hand containing five cards of the same suit, not all of sequential rank. Flush hands are ranked first by the rank of their highestranking card, then by the second highest-ranking card, and so forth. For example, AsJs8s5s2s ranks higher than KsQsJs8s5s, AsTs9s5s2s, or AsJs8s4s3s, and lower than AsJs8s5s3s. Flush hands tie only if the players are playing the board, i.e. the community cards form a flush, which cannot be improved by the hole cards from any player. It is not possible to have flush hands of different suits in a single game.

Straight: Straight is a hand containing five cards of sequential rank, not of the same suit. Similar to straight flush, straights are ranked by the rank of their highest-ranking card, regardless of the suit. Thus, Ts9c8h7d6c ranks the same as Th9c8h7d6h, higher than $8 \mathrm{~h} 7 \mathrm{~d} 6 \mathrm{c} 5 \mathrm{~d} 4 \mathrm{~d}$, and lower than QhJdTs9c8h. The best possible straight, a.k.a. the Broadway, consists of ace to ten of two or more suits, e.g. AsKsQhJhTc. The lowest-ranking straight, a.k.a. the wheel, consists of five to ace of two or more suits, e.g. $5 \mathrm{~d} 4 \mathrm{c} 3 \mathrm{~s} 2 \mathrm{hAd}$. 
Three of a Kind: Three of a kind is a hand containing three cards of the same rank and two cards from two other ranks. Hands within this category are ranked first by their triplet, then by the rank of the higherranking kicker, and finally by the lower-ranking kicker. For example, JsJdJcTs8c ranks the same as JsJhJdTh8c, higher than TsThTdsAsJs, and lower than JsJdJcAs2c or JsJdJcTs9h.

Two Pair: Two pair is a hand containing two cards of the same rank, two cards of another rank, and one card from a third rank. Two pair hands are compared first by the rank of the higher-ranking pair, then by the rank of the lower-ranking pair, and finally by the kicker. For example, AsAcTsTcQh is ranked higher than KhKdQsQhAs, and lower than AsAhQhQcTc or AsAhTsTcKc.

One Pair: One pair is a hand containing two cards of the same rank and three cards of three other ranks. Hands in this category are ranked first by the rank of the pair, then by the rank of the kickers from highest to the lowest. Therefore, AsAcKhTc7h ranks higher than KsKhAsTc7h, AsAcQsJdTc or AsAcKhTc2d.

High Card: High card, a.k.a. no-pair or nothing, is a hand containing five cards not all of sequential rank or of the same suit, and none of which are of the same rank. Hands in this category are ranked first by their highest-ranking card, then the second-highest-ranking card, and so forth. Thus, AsJh8s5d2s ranks higher than KcQsJh8s5d, AsTs9h5d2s, or AsJh8s4d3c, and lower than AsJh8s5d3s. 
The game rules in the previous section and the above rankings of hands define the imperfect information game studied in this dissertation.

\subsection{RELATED TERMINOLOGY}

This section introduces some common poker terms used in this dissertation.

Bluff and Semi-bluff: A bluff is a raise (bet) or all-in for the purpose of inducing a fold from the opponent. Usually, the bluffer is holding a hand that is (believed to be) unlikely to win in a showdown. In a semi-bluff, while the bluffer's hand is unlikely to win at the time of the bluff, it is possible to be improved by community cards dealt in future betting rounds (thus becoming the winning hand in a showdown).

As an example, suppose a player holds Qs7s, and the board contains AsTs6h2c, the player bets $\$ 300$ to a $\$ 400$ pot. In this case, the player's move is a semi-bluff. While the player holds only a high card hand now, if a card of spades falls on the river, the player's hand can be improved to a flush, which is very likely to win.

Board Texture: Board texture refers to the conditions of the community cards, e.g. whether the board contains pair(s), three or more suited cards, connected cards, etc. Such conditions are important in evaluating the strength of a player's hand before showdown, because they make certain high-ranking hands possible.

A pair on the board makes four of a kind and full house possible. Three or more suited community cards make flush possible. Players are likely to showdown with a straight on a highly connected board, e.g. QhJdTs (flop), Ts8h7c6s5d (river), etc. 
Note that conditions such as two suited or connected cards on the flop are also important board textures, for the turn card and/or the river card may complete big hands.

A board is considered " $d r y$ " if few such conditions exist, and "wet" otherwise. It is more difficult to evaluate the strength of a hand on a wet board than on a dry board, for wet boards make high-ranking hands such as straight, flush, full house, and/or quads possible, and more conditions must be taken into consideration.

Check-raise: Check-raise is a tactic used by poker players postflop. As an example, player A checks the flop, player B bets $\$ 200$ to the $\$ 400$ pot, making the pot $\$ 600$, and player $A$ then makes a $\$ 1000$ raise (first check, then raise). By check-raising, player A is representing a big hand. This tactic can be used to induce a bet from the opponent or as a bluff.

Draw: A draw is a situation in which a player's hole cards cannot form a strong hand given the board now, but may become the best hand as more community cards are dealt. A player's hand has high drawing power if many cards in the deck can make that hand the best.

As an example, a common type of draws is the flush draw, e.g. the player holds AhKh, and the flop goes $8 \mathrm{~h} 7 \mathrm{~h} 2 \mathrm{~s}$. In this case, any card of hearts will improve the player's hand to an ace-high flush, which is likely to be the best hand in a showdown. Another common type of draws is the straight draw. As an example, the flop goes $9 \mathrm{c} 8 \mathrm{~h} 5 \mathrm{~d}$, and a player holds JsTd. In this case, a queen or a seven of any suit will improve the player's hand to a straight. 
Hand strength: In this dissertation, hand strength refers to the probability of a player's hand being the winning hand if the game goes into a showdown. The hand strength depends on the hole cards as well as the board and may change drastically as more community cards are dealt. Note that incomplete observation makes it rather difficult to compute the real hand strength. However, hand strength can be estimated based on the hole cards, the board, the sequence of actions in the current game, and the history of previous games against the same opponent.

n-bet: $n$-bet, e.g. 3-bet, 4-bet, etc, refers to a raise. Conventionally, the number before the hyphen is the count of raises, including the action itself. In the preflop betting round, the big blind is considered the first raise (1-bet), if player A raises (2-bet), and player B raises after player A, player B's move is called a 3-bet. If player A raises again after player $\mathrm{B}$, that move is called a 4-bet.

The concept of $n$-bet can also be used to describe postflop raises. If player A raises the flop (1-bet), player B raises after player A (2-bet), and player $\mathrm{A}$ raises again after player $\mathrm{B}$, the last move is a 3-bet.

Pot odds: Pot odds is the minimum probability of hand winning in a showdown that justifies calling a raise from the opponent, assuming that the game will go to a showdown without additional chips being committed to the pot. Pot odds op can be computed as:

$$
o_{\mathrm{p}}=\frac{x}{x+s_{\mathrm{p}}}
$$

where $x$ is the amount of chips the player has to commit for the call, and $S_{\mathrm{p}}$ is the size of the pot (before calling). 
Value bet: A bet from a player holding a strong hand with the purpose of inducing a call.

$x$-pot raise: $x$-pot raise, e.g. 1/4-pot raise, half-pot raise, pot-size raise, etc, denotes a raise (or bet) of a certain size. Note that (1) the size of the raise is equal to the amount of chips committed by the player after the raise minus the amount of chips committed by the opponent so far (as is defined in Section 2.1), and (2) the "pot" in "x-pot raise" refers to the size of the pot after the players match their contribution.

As an example, suppose player A (as the button) makes a pot-size raise preflop on a $\$ 50 / \$ 100$ table. That is, player A first calls the big blind, making the pot $\$ 200$, and then raises by the size of that pot, i.e. $\$ 200$. As a result, after this action, player A will commit $\$ 300$ into the pot, and the pot has a total $\$ 400$. Suppose player B calls, making the pot $\$ 600$. On the flop, player B makes a half-pot raise. In this case, player B will commit an extra $\$ 300$ (half of the pot) to the pot since both players have already matched their contributions. Assuming player A responds by a 3/4-pot raise, similar to the preflop raise, this means that player A first matches the contribution of player B, i.e. call with $\$ 300$, making the pot $\$ 1200$, and then raises by $3 / 4$ of that pot, i.e. committing another $\$ 900$. By the end of this action, the pot is $\$ 2100$ with $\$ 1500$ from player A.

As a table game, HUNL is easy to model mathematically. Its enormous game state space presents a great challenge for building high-performance agents. In addition, it is a two-player version of arguably the most popular variant of poker enjoyed by millions of 
players around the world. Thus, HUNL has become the most extensively studied problems for research on imperfect information games in recent years. 


\section{Chapter 3: Related Work}

This Chapter introduces related work on imperfect information games and poker in particular. Section 3.1 discusses the state-of-the-art approach in building computer poker agent, i.e. Nash Equilibrium Approximation. Section 3.2 presents related work on opponent modeling and exploitation in poker. Section 3.3 introduces recurrent neural networks and neuroevolution and summarizes the motivation for applying such techniques in building adaptive poker agents.

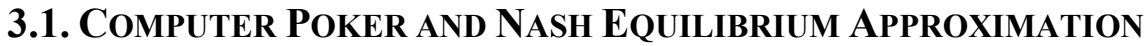

Poker has been studied academically since the founding of game theory. In fact, the only motivating problem described by Nash [1951] in his Ph.D. thesis, which defined and proved existence of the central solution concept, was a three-player poker game. Ever since then, poker has been one of the most visible applications of research in computational game theory, and building poker agents following Nash equilibrium strategy or approximated Nash-equilibrium strategy has been considered a mainstream direction for research on computer poker.

The Nash equilibrium approximation approach is based on the existence of optimal strategies, or Nash equilibria in the target game. Using such strategies ensures that an agent will obtain at least the game-theoretic value of the game, regardless of the opponent's strategy [Billings et al. 2003]. For two-player zero-sum games such as heads-up poker, Nash equilibria are proven to exist. Furthermore, the game value of such strategies in heads-up poker is zero [von Neumann, 1928]. Thus, if a real Nash equilibrium strategy for heads-up poker is found, that strategy is guaranteed to either win or tie against any opponent in the long run. 
This section introduces the related work on computer poker and Nash equilibrium approximation. Subsection 3.1.1 outlines existing work on small-scale poker variants. Subsection 3.1.2 focuses on equilibrium approximation and abstraction techniques, which are applied to full-scale poker, and Subsection 3.1.3 summarizes the related work and points out directions for improvement.

\subsubsection{Simple Poker Variants}

The biggest challenge for applying the Nash equilibrium approximation approach to full-scale poker games is the enormous size of their state space. For instance, Heads-Up Limit Texas Holdem has over $10^{17}$ game states, and HUNL as in the format adopted by the ACPC in recent years has more than $10^{164}$ game states. Therefore, early studies on poker usually focused on finding Nash equilibrium strategies for simplified poker games whose equilibria could be manually computed [Kuhn, 1950; Sakaguchi and Sakai, 1992]. While these methods are of theoretical interest, they are not feasible for full-scale poker [Koller and Pfeffer, 1997].

As computers became more powerful, researchers started to study poker games of bigger scale. Selby [1999] computed an optimal solution for the abbreviated game of preflop Holdem. Takusagawa [2000] created near-optimal strategies for the play of three specific Holdem flops and betting sequences. To tackle more challenging poker variants, abstraction techniques were developed to reduce the game state space while capturing essential properties of the real domain. Shi and Littman [2001] investigated abstraction techniques using Rhode Island Holdem, a simplified variant of poker with over three billion game states. Gilpin and Sandholm [2006b] proposed GameShrink, an abstraction algorithm based on ordered game isomorphism. Any Nash equilibrium in an abstracted smaller game obtained by applying GameShrink to a larger game can be converted into an equilibrium 
in the larger game. Using this technique, a Nash equilibrium strategy was computed for Rhode Island Holdem [Gilpin and Sandholm, 2005].

\subsubsection{Full-scale Poker}

Billings et al. [2003] transformed Heads-Up Limit Texas Holdem into an abstracted game using a combination of abstraction techniques such as bucketing, preflop and postflop models, betting round reduction and elimination. Linear programming solutions to the abstracted game were used to create poker agents for Heads-Up Limit Holdem, which achieved competitive performance against human players. Billings' work is the first successful application of Nash equilibrium approximation approach to building highquality computer agents for full-scale poker games.

To address the challenge of large-scale imperfect information games such as HeadsUp Holdem more effectively, the Nash equilibrium approximation approach was further improved in two directions: (1) equilibrium approximation algorithms and (2) abstraction techniques.

\section{Equilibrium Approximation Techniques}

The development of equilibrium approximation algorithms focuses on reducing computational cost and improving the quality of approximation. For two-player zero-sum games, there exists a polynomial-time linear programming formulation based on the sequence form such that strategies for the two players correspond to primal and dual variables, respectively. Thus, a minimax solution for small-to-medium-scale two-player zero-sum games can be computed via linear programming [Koller et al. 1996]. This method was extended to compute sequential equilibria by Miltersen and Sorensen [2006]. However, it is generally not efficient enough for large-scale games. 
Hoda et al. [2006] proposed a gradient-based algorithm for approximating Nash equilibria of sequential two-player zero-sum games. The algorithm uses modern smoothing techniques for saddle-point problems tailored specifically for the polytopes in the Nash equilibrium. Shortly after, Gilpin et al [2007a] developed a gradient method using excessive gap technique (EGT).

Heinrich and Silver [2016] introduces the first scalable end-to-end approach to learning approximate equilibrium strategy without prior domain knowledge. This method combines fictitious self-play with deep reinforcement learning. Empirical results show that the algorithm approached an equilibrium strategy in Leduc poker and achieved comparable performance against the state-of-the-art equilibrium strategies in Limit Holdem.

An efficient algorithm for finding Nash equilibrium strategies in large-scale imperfect information games, Counterfactual Regret Minimization (CFR), was introduced by Zinkevich et al. [2007]. CFR minimizes overall regret by minimizing counterfactual regret and computes Nash equilibrium strategy through iterative self-play. Compared to prior methods such as linear programming, CFR is more efficient and can be utilized to solve much larger abstractions.

A series of studies have been dedicated to optimizing and extending CFR in recent years. Lanctot et al. [2009] proposed a general family of domain-independent CFR samplebased algorithms called Monte Carlo CFR (MCCFR). Monte Carlo sampling reduces cost per iteration in MCCFR significantly, leading to drastically faster convergence.

Burch et al. [2014] developed an offline game solving algorithm, CFR-D, for decomposing an imperfect information game into subgames that can be solved independently while retaining optimality guarantees on the full-game solution. This technique can be used to construct theoretically justified algorithms that overcome memory or disk limitations while solving a game or at run-time. 
End-game solving was proposed by Ganzfried and Sandholm [2015a]. This method computes high-quality strategies for endgames rather than what can be computationally afforded for the full game. It can solve the endgame in a finer abstraction than the abstraction used to solve the full game, thereby making the approximated strategy less exploitable. Brown and Sandholm [2017] introduced safe and nested endgame solving, which outperformed prior end game solving methods both in theory and in practice.

In addition, Brown and Sandholm [2014] proposed a warm-starting method for CFR through regret transfer, saving regret-matching iterations at each step significantly. Jackson [2016] proposed Compact CFR to reduce memory cost through a collection of techniques, allowing CFR to be run with $1 / 16$ of the memory required by classic methods.

\section{Abstraction Techniques}

Meanwhile, a number of abstraction techniques were developed to capture the properties of the original game states effectively and efficiently. The basic approach for abstraction in poker is to cluster hands by Expected Hand Strength (EHS), which is the probability of winning against a uniform random draw of private cards for the opponent, assuming a uniform random roll-out of the remaining community cards. Clustering algorithm such as $k$-means were employed to group similar game states together using the difference of EHS as the distance metric [Billings et al. 2003; Zinkevich et al. 2007].

Gilpin and Sandholm [2006a] developed an automatic abstraction algorithm to reduce the complexity of the strategy computation. A Heads-Up Limit Holdem agent, GSI, was built using this technique. GSI solves a large linear program to compute strategies for the abstracted preflop and flop offline and computes an equilibrium approximation for the turn and river based on updated probability of the opponent's hand in real time. The agent was competitive against top poker playing agents at the time. 
While EHS is a reasonable first-order-approximation for the strength of a hand, it fails to account for the entire probability distribution of hand strength. To address this problem, distribution-aware abstraction algorithms were developed. In particular, Gilpin et al. [2007b] proposed potential-aware automated abstraction of sequential games. This technique considers high-dimensional space consisting of histograms over abstracted classes of states from later stages of the game, and thus taking into account the potential of a hand automatically. Abstraction quality is further improved by making multiple passes over the abstraction, enabling the algorithm to narrow the scope of analysis to information that is relevant given abstraction decisions made earlier.

Ganzfried and Sandholm [2014] proposed an abstraction algorithm for computing potential-aware imperfect recall abstractions using earth mover's distance. Experimental results showed that the distribution-aware approach outperforms EHS-based approaches significantly [Gilpin and Sandholm, 2008b; Johanson et al. 2013].

\subsubsection{Conclusions on Nash Equilibrium Approximation}

Progress in both equilibrium-approximation algorithms and abstraction techniques has led to remarkable successes in building high-performance agents for full-scale poker using the Nash equilibrium approximation approach. Heads-Up Limit Texas Holdem has been solved [Bowling et al. 2015; Tammelin et al. 2015]. In recent Human vs. AI competitions, Nash-equilibrium-based computer agents such as Libratus and DeepStack have defeated top professional human players in HUNL with statistically significant margins [Moravcik et al., 2017; Brown and Sandholm, 2017].

For reasons discussed in the Chapter 1, most recent work on computer poker largely focuses on HUNL. While the Nash equilibrium approximation approach has been successful, studies also revealed that the approach has several important limitations. In 
particular, Lisy and Bowling [2016] showed that most top-ranking poker agents for HUNL built through this approach are exploitable with a simple local best-response method. The enormous state space of HUNL also renders abstraction and solving abstracted games rather costly [Brown and Sandholm, 2017]. Performance of the equilibrium approximation approach is not guaranteed in games with more than two players and multiple equilibria [Ganzfried, 2016]. In addition, agents following equilibrium strategy lack the ability to adapt to their opponents and are unable to exploit the opponent's weaknesses effectively [Li and Miikkulainen, 2017].

Therefore, as is pointed out in the introduction, developing new methods in building high-performance computer agent for HUNL that is adaptive and does not rely on Nash equilibrium is an interesting and promising direction for future research in imperfect information game.

\subsection{OPPONENT MODELING AND EXPLOITATION}

While a real Nash equilibrium strategy is statistically unexploitable, it is unlikely to be the most profitable strategy. The most effective strategy against each opponent is different, and only through opponent modeling and adaptation, can a player approximate such counter-strategies for all opponents [ $\mathrm{Li}$ and Miikkulainen, 2018]. Hence, to achieve high performance in an imperfect information game such as Texas Holdem, the ability to model and exploit suboptimal opponents effectively is critical [Billings et al. 2002].

\subsubsection{Non-equilibrium-based Opponent Exploitation}

Similar to the Nash equilibrium approximation approach, the research on opponent modeling in poker started with relatively simple variants such as Stud Poker and Limited Holdem. This subsection outlines the early work on opponent modeling and exploitation. 


\section{Rule-based Statistical Models}

The first attempts to construct adaptive poker agents employed rule-based statistical models. Billings et al. [1998] described and evaluated Loki, a poker program capable of observing its opponents, constructing opponent models and dynamically adapting its play to exploit patterns in the opponents' play. Loki models its opponents through weighting for possible opponent hands. Weights are initialized and adjusted by hand-designed rules according to actions of the opponent. Both generic opponent modeling (i.e. using a fixed strategy as a predictor) and specific opponent modeling (i.e. using an opponent's personal history of actions to make predictions) were evaluated. Empirical results in Limit Texas Holdem showed that Loki outperformed the baseline system that did not have the opponent model. In addition, experimental results showed that specific opponent modeling was generally more effective than generic opponent modeling.

Billings et al. [2002] presented an upgraded version of the rule-based poker agent, Poki, which adopts specific opponent modeling and constructs statistical models for each opponent. The opponent model is essentially a probability distribution over all possible hands. It is maintained for each player participating in the game, including Poki itself. The opponent modeler uses the hand evaluator, a simplified rule-based betting strategy, and learned parameters about each player to update the current model after each opponent action. The work also explored the potential of neural-network-based opponent models. The result is a program capable of playing reasonably strong poker, but there remains considerable research to be done to play at a world-class level in Heads-Up Limit Texas Holdem.

Billings et al. [2006] proposed opponent modeling with adaptive game tree algorithms: Miximax and Miximix. The algorithms compute the expected value at decision nodes of an imperfect information game tree by modeling them as chance nodes with 
probabilities based on the information known or estimated about the domain and the specific opponent. The algorithm performs a full-width depth-first search to the leaf nodes of the imperfect information game tree to calculate the expected value of each action. The probability of the opponent's possible actions at each decision node is based on frequency counts of past actions. The expected value at showdown nodes is estimated using a probability density function over the strength of the opponent's hand, which is an empirical model of the opponent based on the hands shown in identical or similar situations in the past. Abstraction techniques were used to reduce the size of the game tree. The poker agents using these methods, Vexbot, outperformed top-ranking agents at the time in Heads-Up Limit Holdem (e.g. Sparbot, Hobbybot, Poki, etc).

\section{Bayesian Models}

A second direction of research in opponent modeling is Bayesian model. Korb et al. [1999] developed the Bayesian Poker Program (BPP). The BPP uses a Bayesian network to model the program's poker hand, the opponent's hand, and the opponent's actions conditioned upon the hand and the betting curves. The history of play with opponents is used improve BPP's understanding of their behavior. Experimental results showed that the BPP outperformed simple poker agents that did not model their opponents as well as nonexpert-level human players in five-card-stud poker, a relatively simple variant of the game.

Later on, Southey et al. [2005] proposed a Bayesian probabilistic model for a broad class of poker games, fully modeling both game dynamics and opponent strategies. The posterior distribution was described and several approaches for computing appropriate responses considered, including approximate Bayesian best response, Max A Posteriori (MAP), and Thompson's response. Independent Dirichlet prior was used for Leduc Holdem and expert-defined informed prior for Limit Texas Holdem. Experimental results 
in Leduc Holdem and Heads-Up Limit Texas Holdem showed that the model was able to capture opponents drawn from the prior rapidly and the subsequent responses were able to exploit the opponents within 200 hands.

Further, Posen et al. [2008] proposed an opponent modeling approach for No-Limit Texas Holdem that starts from a learned prior (i.e., general expectations about opponent behavior) and learns a relational regression tree to adapt to these priors to specific opponents. Experimental results showed that the model was able to predict both actions and outcomes for human players in the game after observing 200 games, and that in general, the accuracy improves with the size of the training set. However, the opponent models were not integrated into any poker agents, and the performance of agents using these models in Heads-Up No-Limit Holdem against human players or other computer agents remains unclear.

\section{Neural Networks}

A third direction for opponent modeling in poker is neural networks. They can be either used to building opponent modeling components or complete agents. Davidson et al. [2000] proposed to use neural networks to assign each game state with a probability triple, predicting the action of the opponent in Heads-Up Limit Texas Holdem. The prediction had an accuracy of over $80 \%$. Billings et al. [2002] integrated neural-network-based predictors into the Poki architecture and showed that the neural network predictors outperformed simple statistical models. Lockett and Miikkulainen [2008] proposed a method to evolve neural networks to classify opponents in a continuous space. Poker agents were trained via neuroevolution both with and without the opponent models, and the players with the models conclusively outperformed the players without them. 


\section{Miscellaneous Techniques}

A few other methods for opponent modeling were explored in addition to these three main approaches. Teofilo and Reis [2011] presented an opponent modeling method based on clustering. The method applies clustering algorithms to a poker game database to identify player types in No-Limit Texas Holdem based on their actions. In the experiments, the opponents were clustered into seven types, each having its own characterizing tactics. However, the models were not used to build poker agents or tested in real matches.

Ensemble learning was introduced for opponent modeling in poker by Ekmekci and Sirin [2013]. This work outlines a learning method for acquiring the opponent's behavior for the purpose of predicting opponent's future actions. A number of features were derived for modeling opponent's strategy. Expert predictors were trained to predict actions of a group of opponents, respectively. An ensemble learning method was used for generalizing the model based on these expert predictors. The proposed approach was evaluated on a set of test scenarios and shown to be effective in predicting opponent actions in Heads-Up Limit Texas Holdem.

Bard et al. [2013] proposed an implicit modeling framework where agents aim to maximize the utility of a fixed portfolio of pre-computed strategies. A group of robust response strategies were computed and selected for the portfolio. Variance reduction and online learning techniques were used to dynamically adapt the agent's strategy to exploit the opponent. Experimental results showed that this approach was effective enough to win the Heads-Up Limit Holdem opponent exploitation event in the 2011 ACPC.

\subsubsection{Equilibrium-based Opponent Exploitation}

While the above work lays a solid foundation for research on opponent modeling in poker, computer poker agents using none of these models demonstrated competitive 
performance in HUNL. In fact, most of the above models were not integrated with any poker agent architecture, and their performance was not evaluated against computer or human opponent in heads-up matches of any variants of poker.

As introduced in Section 3.1, the Nash equilibrium approximation approach has become the mainstream method in recent years for building poker agents for both Limit and No-Limit Holdem. While being relatively difficult to exploit, poker agents following approximated equilibrium strategies lack the ability to model and exploit their opponents. Therefore, researchers have attempted to combine opponent modeling with equilibrium approximation.

In particular, Ganzfried and Sanholm [2011] proposed an efficient real-time algorithm that observes the opponent's action frequencies and built an opponent model by combining information from an approximated equilibrium strategy with the observations. It computes and plays a best response based on the opponent model, which is updated continually in real time. This approach combines game-theoretic reasoning and pure opponent modeling, yielding a hybrid that can effectively exploit opponents after only a small number of interactions. Experimental results in Heads-Up Limit Texas Holdem showed that the algorithm leads to significantly higher win rates against a set of weak opponents. However, the agent built in this method may become significantly exploitable to strong opponents.

A series of work has been dedicated to reducing exploitability of adaptive agents. Johanson et al. [2008] introduced technique for computing robust counter-strategies for adaptation in multi-agent scenarios under a variety of paradigms. The strategies can take advantage of a suspected tendency in the actions of the other agents while bounding the worst-case performance when the tendency is not observed. 
Johanson and Bowling [2009] proposed data-biased responses for generating robust counterstrategies that provide better compromises between exploiting a tendency and limiting the worst case exploitability of the resulting counter-strategy. Both techniques were evaluated in Heads-Up Limit Holdem and shown effective.

Ponsen et al. [2011] proposed Monte-Carlo Restricted Nash Response (MCRNR), a sample-based algorithm for the computation of restricted Nash strategies. These strategies are robust best-response strategies that are not overly exploitable by other strategies while exploiting sub-optimal opponents. Experimental results in Heads-Up Limit Holdem showed that MCRNR learns robust best-response strategies fast, and that these strategies exploit opponents more than playing an equilibrium strategy.

Norris and Watson [2013] introduced a statistical exploitation module that is capable of adding opponent exploitation to any base strategy for playing No-Limit Holdem. The module is built to recognize statistical anomalies in the opponent's play and capitalize on them through the use of expert designed statistical exploitation. Such exploitation ensures that the addition of the module does not make the base strategy more exploitable. Experiments against a range of static opponents with varying exploitability showed promising results.

Safe opponent exploitation was proposed by Ganzfried and Sandholm [2015b]. A full characterization of safe strategies was presented and efficient algorithms developed for exploiting sub-optimal opponents while guaranteeing safety. Experimental results in Kuhn poker showed that (1) aggressive safe exploitation strategies significantly outperform adjusting the exploitation within equilibrium strategies, and (2) all the safe exploitation strategies significantly outperform non-safe best response strategy against strong dynamic opponents. 
While these techniques improve adaptive agents' performance against high-quality equilibrium-based opponents, they also restrict adaptation and are unable to fully exploit relatively weak opponents. The idea of "safety" is meaningful only if the agent is unable to adapt to the opponent quickly enough to exploit its strategy. An agent with ideal opponent modeling should be able to adapt its strategy to approximate the best response against all opponents. In other words, it should aim for the maximum exploitation against every opponent, weak or strong, and rely on adaptation to avoid being exploited rather than restricting adaptation to limited deviation from equilibrium strategies.

\subsubsection{Conclusions on Opponent Modeling}

As discussed in the previous two subsections, the research on opponent modeling and exploitation in poker is relatively limited. Most of the techniques were applied to smallscale poker variants or tested as a separate component without being integrated into poker agents. While latest work has started tackling more complex poker variants such as HUNL, they are generally equilibrium-based and cannot exploit flawed opponents effectively. Therefore, it is an interesting and rewarding challenge to develop new opponent modeling and exploitation method that is effective in No-limit Holdem and other large-scale imperfect information games.

\subsection{LSTM AND NEUROEVOLUTION}

As pointed out in the previous section, neural networks have been used in opponent modeling, and have already yielded promising results in Limit Texas Holdem. Existing work employed only simple feed-forward neural networks. However, decision-making in many large-scale imperfect information games should be based on not only the current observation but also observations in the past. 
In the case of HUNL, a players should consider previous observations in two levels. Action sequence of both players in the current game is crucial for evaluating opponent hand strength and predicting future actions. In addition, history of previous games against the same opponent is essential in exposing and exploiting the opponent's strategy.

Therefore, compared to using feed-forward neural networks in opponent modeling, recurrent neural networks are preferable for their ability to extract and balance information over time. This section reviews related work on LSTM and neuroevolution, which can be utilized to model opponents effectively.

\subsubsection{LSTM Neural Networks}

Hochreiter and Schmidhuber [1997] proposed a type of recurrent neural network, Long Short Term Memory (LSTM), which was later improved by Gers et al. [2000]. A LSTM network is a neural network that contains LSTM blocks instead of, or in addition to, other network units. A LSTM block is a recurrent network module that excels at remembering values for either long or short duration of time. The key to this ability is that it uses no activation function within its recurrent components. Therefore, the stored value is not iteratively squashed over time, and the gradient does not tend to vanish when backpropagation through time is applied for training.

Figure 3-1 illustrates the structure of a vanilla LSTM block. The LSTM blocks memorizes and accumulates information with cell states. The input gate filters the input, the forget gate discards outdated or irrelevant information, and the output gate may strengthen or block the cell states before sending them as the output. Note that the outputs of the three gates are influenced by the current input, the previous output, and the cell states if a peephole connection exists. 


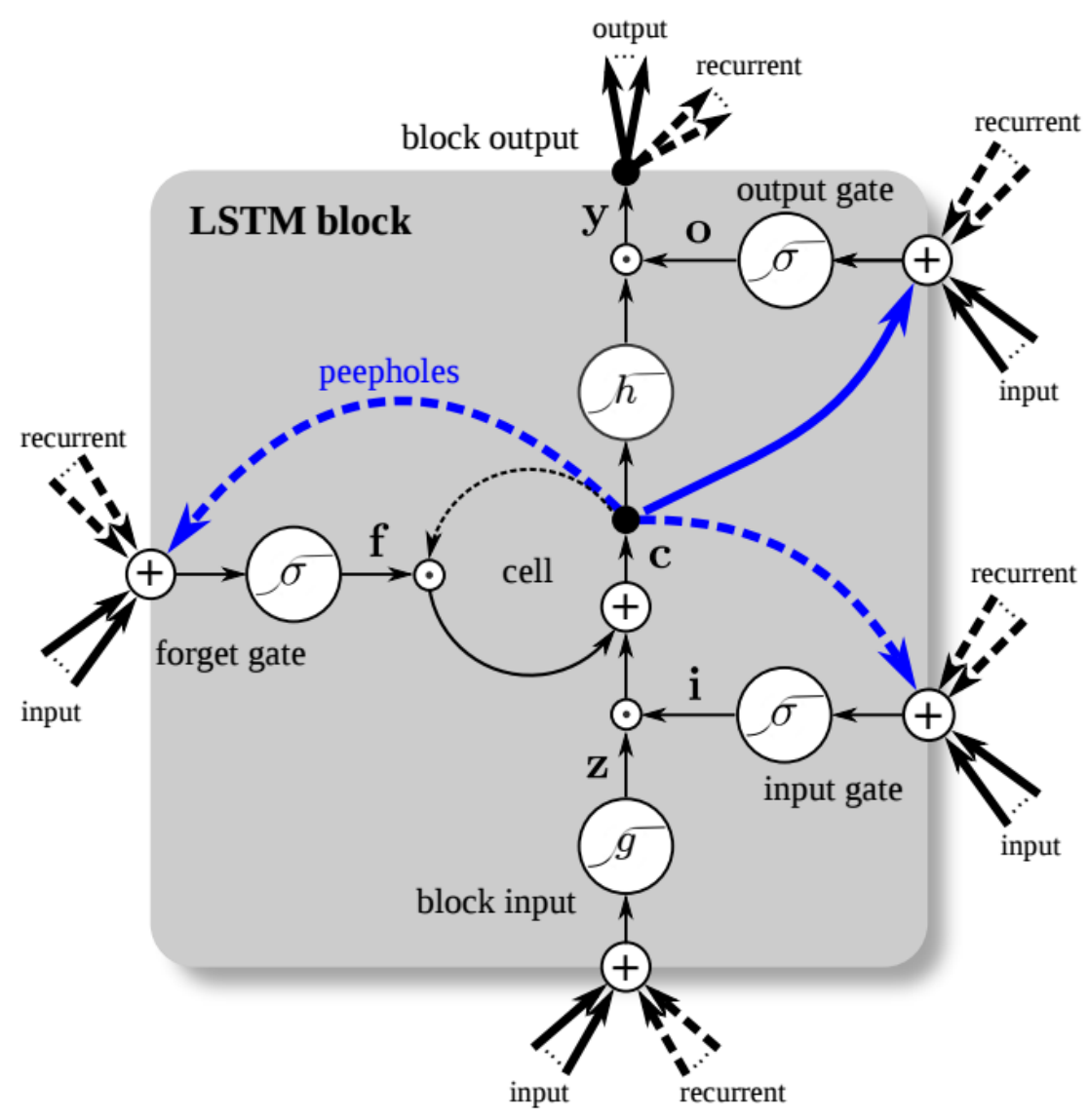

Figure 3-1: A Vanilla LSTM Block. The vanilla LSTM blocks are the basic functional unit in the LSTM modules. A block consists of three gates (input, forget, and output), a block input, a cell, an output activation function, and peephole connections. The outputs are sent back to the block input and all gates via recurrent connections.

The vector formulas for a Vanilla LSTM block forward pass are:

$$
\begin{array}{rr}
\mathbf{z}_{t}=g\left(\mathbf{W}_{\mathrm{z}} \mathbf{x}_{t}+\mathbf{R}_{\mathrm{z}} \mathbf{y}_{t-1}+\mathbf{b}_{\mathrm{z}}\right) & \text { block input (1) } \\
\mathbf{i}_{t}=\sigma\left(\mathbf{W}_{\mathrm{i}} \mathbf{x}_{t}+\mathbf{R}_{\mathrm{i}} \mathbf{y}_{t-1}+\mathbf{p}_{\mathrm{i}} * \mathbf{c}_{t-1}+\mathbf{b}_{\mathrm{i}}\right) & \text { input gate (2) } \\
\mathbf{f}_{t}=\sigma\left(\mathbf{W}_{\mathrm{f}} \mathbf{x}_{t}+\mathbf{R}_{\mathrm{f}} \mathbf{y}_{t-1}+\mathbf{p}_{\mathrm{f}} * \mathbf{c}_{t-1}+\mathbf{b}_{\mathrm{f}}\right) & \text { forget gate (3) } \\
\mathbf{c}_{t}=\mathbf{i}_{t} * \mathbf{z}_{t}+\mathbf{f}_{t} * \mathbf{c}_{t-1} & \text { cell state (4) } \\
\mathbf{o}_{t}=\sigma\left(\mathbf{W}_{\mathrm{o}} \mathbf{x}_{t}+\mathbf{R}_{\mathrm{o}} \mathbf{y}_{t-1}+\mathbf{p}_{\mathrm{o}} * \mathbf{c}_{t}+\mathbf{b}_{\mathrm{o}}\right) & \text { output gate (5) } \\
\mathbf{y}_{t}=\mathbf{o}_{t} * h\left(\mathbf{c}_{t}\right) & \text { block output (6) }
\end{array}
$$

where $\mathbf{x}_{t}$ is the input vector at time $t$, the $\mathbf{c}$ are the cell vectors, the $\mathbf{y}$ are the output vectors. Note that the dimension of the cell vectors is equal to that of the output vectors. For 
convenience of discussion, it is referred to as the cell size. The $\mathbf{W}$ are weight matrices for the inputs, the $\mathbf{R}$ are recurrent weight matrices for the outputs, the $\mathbf{p}$ are peephole weight vectors, and the $\mathbf{b}$ are bias vectors. The asterisks denote point-wise multiplication of two vectors, and $\sigma, g$, and $h$ are point-wise activation functions. In this application, all LSTM blocks use hyperbolic tangent as both input and output activation function

In recent years, LSTM neural networks have emerged as the state-of-the-art models for a number of challenging AI problems where extraction and analysis of sequential patterns are essential, including handwriting recognition [Doetsch et al., 2014; Graves et al., 2008], language modeling [Zaremba et al., 2014] and translation [Luong et al., 2014], acoustic modeling of speech [Sak et al., 2014], speech synthesis [Fan et al., 2014], and analysis of audio [Marchi et al., 2014], etc. Hence, applying LSTM neural networks for opponent modeling in poker is a promising new direction.

Greff et al. [2015] presented a large-scale analysis of eight LSTM variants on three representative tasks: speech recognition, handwriting recognition, and polyphonic music modeling. Experimental results showed that none of the variants can improve upon the standard LSTM architecture significantly, and that the forget gate and the output activation function to be the most critical components.

This work provides helpful insights for the design and optimization of the architecture of opponent models based on LSTM neural networks.

\subsubsection{Neuroevolution}

A potential difficulty in building LSTM-based opponent models in HUNL is the lack of training data for supervised learning. In particular, the correct outputs of certain predictions cannot be easily obtained (e.g. what is the probability of a certain opponent folding to a pot-size bet given current game state and the history of previous game against 
the same opponent?). Note that even if the opponent's strategy defines the probability distribution of actions explicitly, such distribution, if used directly, may not be a sensible label for the predictions of the opponent model: the distribution is based on information inaccessible to the agent, i.e. the private cards of the opponent. Therefore, an alternative training method is needed.

Figure 3-2 illustrates the framework of genetic algorithms. Such algorithms can be specified in three parts: representation, selection, and reproduction. Agent performance can be evaluated based on overall fitness (e.g. average earnings in poker). Thus, labels for each prediction is unnecessary.

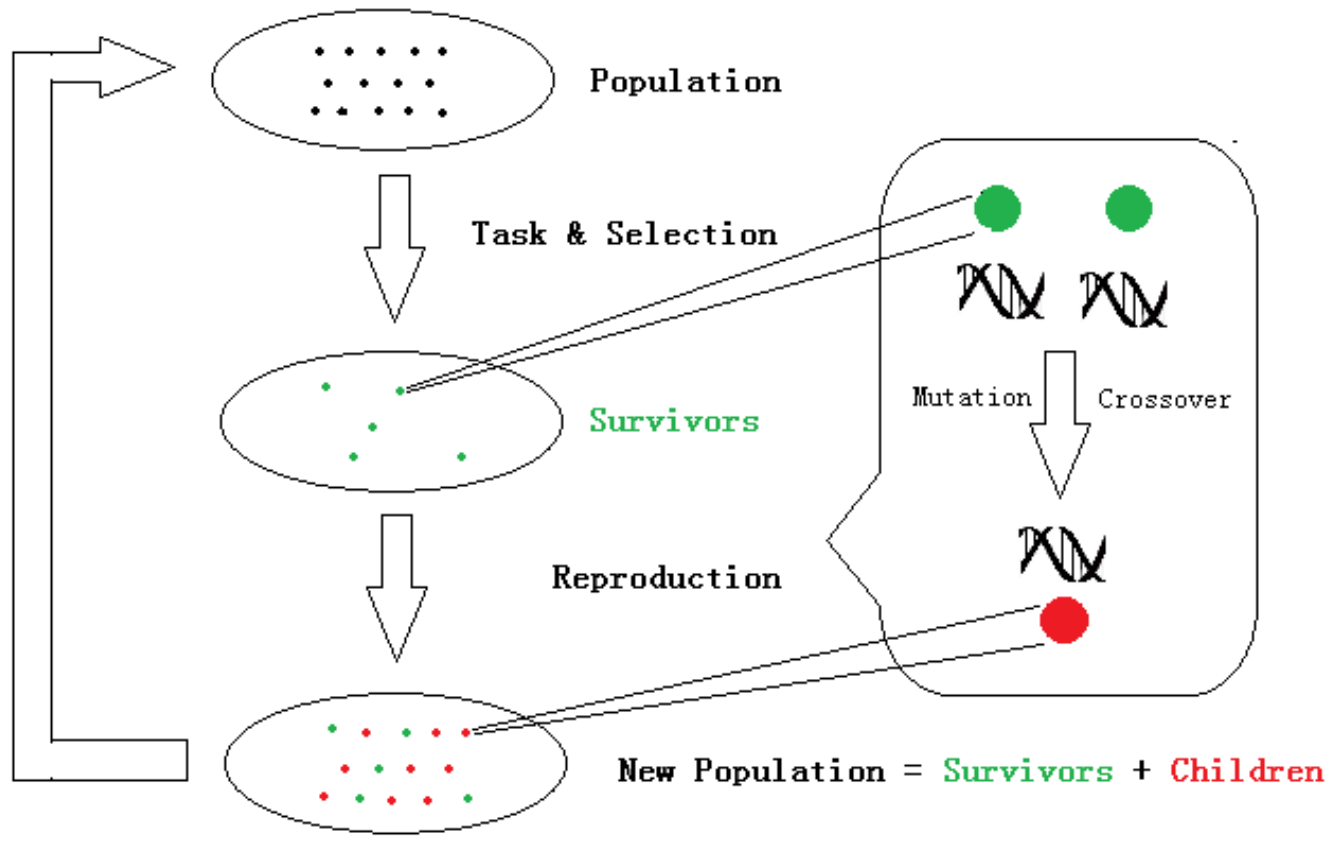

Figure 3-2: Genetic Algorithm Framework. A genetic algorithm optimizes the population iteratively. In each generation, agents perform their tasks and are ranked by their fitness. The agents with the highest fitness survive (selection) and reproduce. Children are generated through mutation and crossover of survivor genomes. The children replace the low-performance agents, and a new generation begins. Thus, the overall fitness of the population increases as evolution proceeds. 
Neuroevolution (NE), the artificial evolution of neural networks using genetic algorithms, has showed great promise in many complex reinforcement learning tasks since its inception [Gomez and Miikkulainen, 1999; Gruau et al., 1996; Moriarty and Miikkulainen, 1997; Potter et al., 1995; Whitley et al., 1993].

In particular, Stanley and Miikkulainen [2002] proposed Neuroevolution with Augmented Topology (NEAT) to evolve neural network topologies along with weights. The algorithm was extended for evolving neural networks in real time [Stanley et al. 2005]. In addition, NE algorithms were proposed to evolve deep unsupervised convolutional network [Koutnik et al. 2014] and multi-modal neural networks [Li and Miikkulainen, 2014; Schrum and Miikkulainen, 2014].

While NEAT was initially designed to evolve feed-forward neural networks and simple recurrent neural networks, its principles for crossover of different topologies, protecting structural innovation using speciation, and growing incrementally from minimal structure can be adjusted for optimizing the architecture of LSTM neural network. Bayer et al. [2009] proposed to optimize LSTM memory cell structure through multi-objective evolutionary algorithm. Rawal and Miikkulainen [2016] extended NEAT for evolving deep LSTM neural networks by introducing information stored in the LSTM networks as a secondary optimization objective.

NE searches for behaviors with highest fitness instead of a specific function. In the context of HUNL, fitness can be evaluated by the agents' average winning in matches against the opponents. Thus, it can be used to evolve LSTM-based opponent models without labeled training data. 


\subsubsection{Conclusions}

Based on the above related work, the proposed dissertation aims at (1) developing an evolutionary method for discovering opponent model based on LSTM neural networks and (2) building high-performance adaptive poker agents using such models. Poker agents built in this method should achieve comparable performance against state-of-the-art equilibrium based agents. In addition, they should be able to adapt to their opponents dynamically, even to those with stronger strategies than they have seen in training, and exploit weak strategies effectively.

Compared to existing method, the proposed approach has several advantages. First, this approach aims at maximizing utility rather than minimizing exploitation, potentially producing agents with higher performance practically. Second, agents built in this method are not bound by Nash-equilibrium strategies and can adapt their moves to exploit opponent's weaknesses more effectively. Third, the proposed approach does not rely on the existence or any property of Nash equilibria in the problem domain and is therefore applicable to a wider range of problems involving imperfect information.

Thus, the approach provides a promising new direction for research in computer poker and imperfect information games. 


\section{Chapter 4: Evolving LSTM-based Opponent Models}

ASHE 1.0 is the first step in devising an evolutionary approach to building recurrent-neural-network-based computer poker agents. It serves three purposes: (1) to validate the general methodology, (2) to devise an effective algorithmic framework that can be used to evolve such agents, and (3) to discover key factors that influence agent performance for further optimization.

This chapter contains four subsections. Section 4.1 details the architecture of the system. Section 4.2 introduces the genetic algorithm to evolve the agents. Section 4.3 presents and analyzes experimental results. Section 4.4 summarizes key findings and conclusions.

\subsection{ASHE 1.0 ARCHITECTURE}

Figure 4-1 illustrates the overall architecture of ASHE 1.0. The opponent module extracts and encodes patterns in opponent strategy based on the entire game history against the opponent, i.e. every action in every hand against the current opponent. In contrast, the game module does not look into previous games. Instead, it focuses on the hand in play and encodes the game states, e.g. action sequence, pot odds, expected hand strength of the agent, etc., of that hand only. Both the opponent and the game module contain a single layer of vanilla LSTM blocks.

The patterns and game states encoded by the LSTM modules are sent to the decision network, whose output is a score indicating the relative strength of the agent's hand compared to the perceived range of the opponent's possible holdings. Finally, the decision algorithm selects the next move for the agent using the score and a set of rules that are specified by human experts. 
The ASHE 1.0 architecture allows the agents to choose actions based on both states in the current game and patterns extracted from previous games against the same opponent. Therefore, agents with this architecture have the potential to adapt their behaviors for different opponents for effective exploitation. The rest of this section introduces the LSTM modules and the decision algorithm in details.

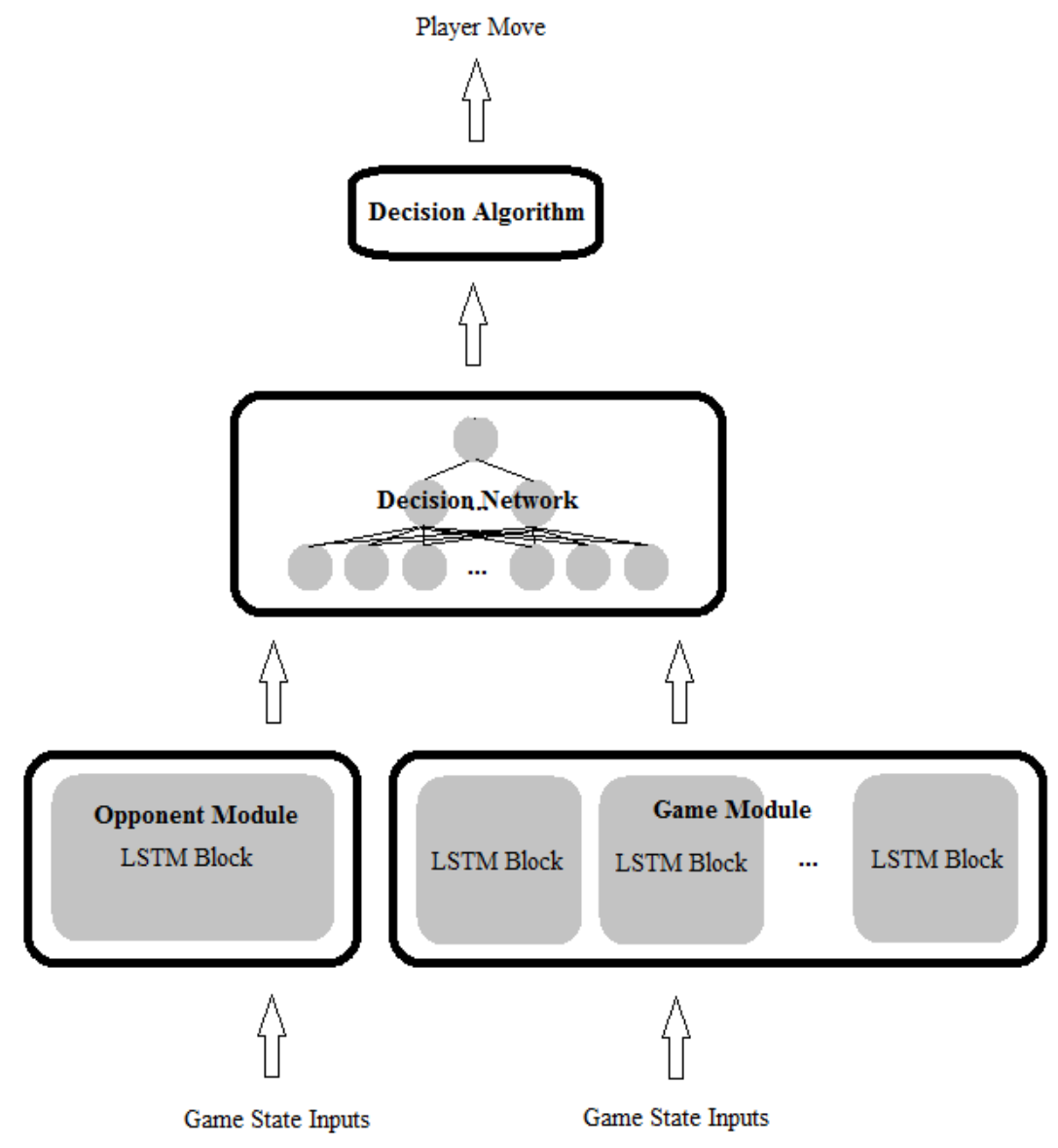

Figure 4-1: ASHE 1.0 Architecture. The system consists of a decision algorithm and three neural network modules: the opponent module, the game module, and the decision network. The opponent module extracts patterns in opponent strategy from game history, and the game module extracts features from the current game. The decision network evaluates relative hand strength based on the outputs from the opponent module and the game module. The decision algorithm selects the agent's action based on the relative hand strength. 


\subsubsection{LSTM Modules}

The LSTM modules enable the agent to extract, memorize, and represent game states and patterns in opponent strategies. Both the game module and the opponent module use vanilla LSTM blocks (as is introduced in Section 3.3) as the basic functional unit.

The game module consists of ten blocks, with a cell size of ten. The game module extracts useful features in the sequence of moves from both players in the current game (hand). Therefore, the blocks in the game module are reset to their initial state for each hand in a heads-up poker match.

The opponent module contains a single block of size fifty. The opponent module is introduced to model the opponent by extracting useful features from the entire history of games played with that opponent. Hence, the blocks in the opponent module are reset for each new opponent rather than each hand.

LSTM blocks in both modules receive inputs in the same format. These inputs area compressed representation of the game state.

1. Current betting round: represented by a one-hot bit vector, each bit corresponding to one of the four stages of the game (i.e. preflop, flop, turn, or river).

2. Expected hand strength: the probability of the agent's hand defeating a random hand in a showdown given the community cards.

3. Chips committed by the opponent: the amount of chips in the pot committed by the opponent so far in the current hand, normalized by initial stack size. Note that in the experiments, stacks are refilled at the beginning of each hand.

4. Chips committed by the player: chips committed by the agent, similar to 3 .

5. Pot odds: as defined in Section 2.3. 
The LSTM blocks are organized into layers and serves as the core component in both LSTM modules, allowing the agent to decide its actions based on game states of the current hand and patterns extracted from previous games against the same opponent.

\subsubsection{Decision Network and Decision Algorithm}

The decision network and algorithm decide the next action of the agent using the outputs from the LSTM modules. The outputs of the LSTM blocks in the game module and the opponent module are concatenated as an input vector to the decision network. The decision network is a fully-connected feed-forward neural network with one hidden layer. In ASHE 1.0, the decision network has a single real valued output $o$, where $-1<o<1$. The output can be considered as an aggression score indicating how aggressive ASHE's next action should be, with -1 and 1 indicating the least and most aggressive, respectively.

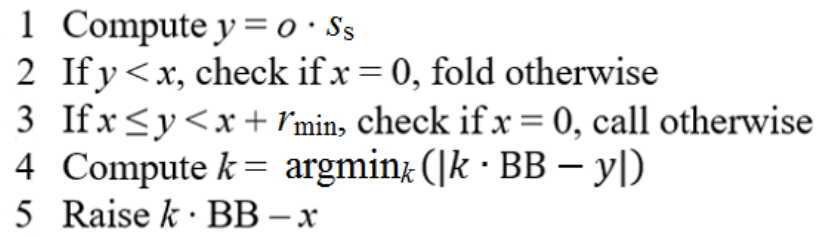

Figure 4-2: ASHE 1.0 Decision Algorithm. The decision algorithm selects the next action for the agent based on the output $o$ from the decision network. The variable $s_{\mathrm{s}}$ is the stack size at the start of the game, $x$ is the amount to call, BB is the big blind, $r_{\min }$ is the minimum raise, and $y$ can be considered as the amount of extra chips the player is willing to commit.

Figure 4-3 presents the decision algorithm in ASHE 1.0. The algorithm uses a straight-forward rule-based approach to decide the next move. If a call is decided but current stack is too short to call, the agent goes all-in instead. In the case of a raise, $k \cdot \mathrm{BB}$ is the amount of extra chips committed to the pot, and $k \cdot \mathrm{BB}-x$ is technically the raise amount. The actual number of chips committed in a raise is converted to integer times of 
the big blind. Similar to the case of a call, if current stack is too short for a decided raise, the player goes all-in. The decision algorithm in ASHE 1.0 takes advantage of opponent modeling through the aggression score, i.e. $o$, which is computed using features extracted by the LSTM modules from both the current and the previous games.

The algorithm has two limitations. First, it does not take possible future actions into consideration. Second, its level of aggression strongly correlates with the relative hand strength. Thus, it is unable to trap the opponent with tactics such as check-raising and slowplaying. However, even with the above limitations, this simplified decision algorithm combined with the LSTM modules and the decision network is sufficient for the agents to demonstrate adaptive behaviors and exploit different opponents, achieving much stronger performance against weak opponents than top-ranking equilibrium-based poker agents.

\subsection{Method of Evolving Adaptive Agents}

This section introduces the genetic algorithm to evolve adaptive RNN-based agents for imperfect information games. Subsection 4.2.1 describes the motivation of using genetic algorithms. Subsection 4.2.2 outlines the algorithmic framework and discusses key technical points.

\subsubsection{Motivation}

While gradient-based approaches for training LSTM neural networks are most commonly used, they require sufficient training samples with correct labels. In poker, the correct label translates into the optimal action for a given game state (i.e. private cards, community cards, action sequence, etc.) and a specific opponent. However, such training data generally do not exist and are infeasible to obtain, because the optimal actions are usually unknown or undefined. Even the best professional players often disagree on what 
the correct move should be when analyzing poker games, especially when the opponent's strategy is taken into consideration.

In contrast, evolutionary methods do not require any labeled training data. The agents can be trained as long as an appropriate fitness function is provided to reflect their overall performance. In other words, the agents are evaluated and optimized based on their overall performance, e.g. in poker, their average earnings, and the value of each action is unnecessary.

A major technical insight in this research is that it is possible to evolve RNN-based agents for imperfect information games through genetic algorithms. This approach allows the method to be applied to problems with little or no labeled training data.

\subsubsection{Evolutionary Method}

Since the Vanilla LSTM blocks in the opponent module and the game module have a fixed structure, and the decision network has a fixed structure as well, the genetic representation of an agent can be constructed by serializing and concatenating weight matrices, weight vectors, bias vectors, etc., of all components.

In order to encourage adaptation, the selection process must allow players to play against opponents with a variety of exploitable strategies. These strategies should require different and preferably opposite counter-strategies for maximum exploitation. Fitness functions should evaluate a player based on its performance against all opponents. In particular, bias must be taken into consideration to prevent experts specialized in exploiting certain types of opponents from constantly dominating the population. For example, exploiting a player who goes all-in every hand can generate far more earnings than exploiting a player who folds to any bets. If the fitness function evaluates agents' performance by simply adding up their earnings against each opponent, the population may 
focus on a few opponents that generate high earnings while ignoring the others, resulting in specialized rather than adaptive behaviors.

Stochasticity in imperfect information games presents an additional challenge to the selection process. For example, the length of game sessions for evaluation must be chosen carefully. If the sessions are too short, high-quality agents are likely to lose due to bad luck. On the other hand, long game sessions may render selection painfully slow. Similarly, a small survival rate may eliminate many unlucky good players, while a large survival rate may preserve poor genotypes and reduce performance of future generations. Furthermore, genomes of adaptive LSTM players tend to be sizable, making it relatively difficult to discover and propagate advantageous genotypes via mutation and crossover.

To alleviate these problems, the Tiered Survival and Elite Reproduction (TSER) method was developed in this dissertation. Survivors of a generation are divided into two tiers based on their fitness. Only members of the top tier, i.e. the elites, are allowed to reproduce. They are immune to mutation as long as they remain in the top tier. Members of the second tier are not allowed to reproduce, neither are they immune to mutation. However, survival provides them the opportunity to preserve advantageous genotypes and improve performance through mutation.

In each generation, all players are evaluated in the same way regardless of their status. Thus, second-tier survivors from previous generations and newborn players in the latest generation can compete for positions in the top tier. After survivors are selected and classified, crossover takes place between randomly selected elites to construct genomes for the next generation. After crossover, children and second-tier survivor genomes are mutated.

TSER has three advantages. First, advantageous genotypes are not lost even if they perform poorly occasionally, because they can survive in the second tier. Second, the 47 
survival rate dilemma no longer exists. A high survival rate coupled with a small top tier can keep poor genotypes from being propagated while reducing accidental losses of desirable genotypes. Third, because advantageous genotypes among elites are immune to mutation, they are preserved for multiple generations, thereby providing them more time to be propagated among entire population.

To balance exploration of genome space and preservation of advantageous genotype, descending mutation rate and strength is introduced. High mutation rate and strength in early stage of evolution allows more effective exploration of the genome space. As evolution proceeds, more advantageous genotypes are discovered, making less aggressive mutation preferable.

The evolutionary method in this section provides an algorithmic framework for evolving adaptive LSTM-based agents for large-scale imperfect information games.

\subsection{EXPERIMENTAL RESULTS}

This section presents experimental results and evaluates the effectiveness of the method outlined in previous sections. Subsection 4.3.1 provides details on experimental setup, including opponents, fitness evaluation, and parameter settings. Subsection 4.3.2 presents and analyzes ASHE 1.0's performance against opponents with high exploitability. Subsection 4.3.3 discusses the performance against Slumbot 2016, a Nash-equilibriumbased player with low exploitability.

\subsubsection{Experimental Setup}

As stated in the previous section, selection and fitness evaluation play a key role in evolution. To encourage adaptation, players need to be evaluated by their performance against multiple opponents with different strategies. Fitness function needs to reward good 
performance against all opponents. Parameter settings need to create a balance between genome space exploration and genotype preservation.

\section{Training Opponents}

Four rule-based players were built as opponents: the Scared Limper, the Calling Machine, the Hothead Maniac, and the Candid Statistician. Their respective strategies are outlined below. The variable $p$ is the winning probability against a random hand given the community cards and the player's private cards.

1 If preflop on button, call the big blind (limp)

2 Compute $o=p^{50}$

3 Decide next move by the Decision Algorithm

a. The Scared Limper. "The ultra-conservative".

1 If check is available, check, otherwise call

b. The Calling Machine. "Call or check only".

1 If opponent limps or checks, raise pot size

2 If raised, re-raise by twice the size of the pot

c. The Hothead Maniac. "Blindly raise".

1 Compute $o=p^{7}$

2 Calculate move by the Decision Algrotihm

d. The Candid Statistician. "Bet honestly".

Figure 4-3: ASHE 1.0 Training Opponents. Four highly exploitable rule-based opponents were used to evolve the agents. To effectively exploit all of these opponents, the agents must demonstrate adaptive behavior and follow different, and in some cases, opposite counter-strategies. 
The Scared Limper always calls the big blind when being the small blind and folds to almost any raise at any stage of the game unless holding top hands (i.e. winning probability close to one). Counter-strategy against the Scared Limper is simple: raise any hand regardless of hand strength; if called or re-raised, fold unless holding the best hand.

The Calling Machine stays in the game for a showdown regardless of hand strength. To effectively exploit its weakness, players must refrain from bluffing and raise aggressively when holding strong hands.

The Hothead Maniac is addicted to bluffing. Similar to the Calling Machine, it is immune to bluffs. The counter-strategy must take pot control into consideration, i.e. it must avoid inflating the pot when holding speculative hand (e.g. a flush draw) and/or moderately strong hands (e.g. a middle pair). Folding becomes necessary when holding weak hands.

The Candid Statistician's moves always reflect its hand strength. Bluffing can be effective when the Candid Statistician holds a weak hand. In addition, the moves from the Candid Statistician can be viewed as an accurate measure of its hand strength, making it much easier for its opponent to take correct actions.

\section{Evaluation and Selection}

In each generation, every LSTM player plays against all four opponents. Two game sessions are played with each opponent. Both sessions contain 500 hands. Card decks in the first session are duplicated and played again in the second session. The players switch seats between the two sessions, and the memory of the LSTM player is cleared before entering the second session. At the beginning of each game, the chip stacks of the two participants are reset to $\$ 20,000$. The big blind of the game is $\$ 100$, and no ante is required. Cumulative earnings (i.e. the total earnings of 1000 hands in two sessions) against every opponent is recorded for all LSTM players. 
To encourage adaptation over specialization, fitness is evaluated based on Average Normalized Earnings (ANE). In the formulas below, $e_{i j}$ is the cumulative earning of player $i$ against opponent $j, m$ is the number of opponents, and $n_{j}$ is the normalization factor for opponent $j$. When the maximum cumulative earning against an opponent is less than one big blind (BB), $n$ is set to BB to avoid normalization errors. Thus, the fitness of the player is computed as:

$$
\begin{aligned}
& f(i)=\mathrm{ANE}_{i}=\frac{1}{m} \sum_{j=1}^{m} \frac{e_{i j}}{n_{j}} \\
& n_{j}=\max \left(\mathrm{BB}, \max _{i}\left(e_{i j}\right)\right)
\end{aligned}
$$

The maximum fitness of a player is 1.0 , which means the player breaks the record of cumulative earnings against every opponent. Note that the cumulative earnings against a single opponent, regardless of its amount, contributes no more than $1 / m$ to the ANE. Thus, normalization makes it more difficult for players specialized in exploiting certain opponent(s) to stay in the top survivor tier.

In the experiments presented by this section, the top tier size is not fixed. Instead, survivors of each generation are ranked according to their ANE, and the survivors whose ANE is above the average of all survivors ascend to the top tier. If the top tier contains only a single player, the second best player is added to the top tier for crossover. This mechanism allows players with genetic breakthroughs to propagate advantageous genotypes quickly.

\section{Parameter Settings}

Table 4-1 presents the values of key parameters. Mutation rate refers to the probability for a gene (i.e. an element in the genome vector) to mutate. Mutation amount follows Gaussian distribution with zero mean, and mutation strength is the standard deviation of the distribution. Mutation rate and strength descend linearly from the initial 
value to the final value as evolution proceeds. Crossover is done by copying elements with odd and even index from the genome vectors of two parents, respectively.

\begin{tabular}{llll}
\hline Parameter & Value & Parameter & Value \\
\hline Number of Generation & 250 & Opponent Module Block & 1 \\
\hline Population Size & 50 & Opponent Module Size & 50 \\
\hline Survival Rate & 0.30 & Game Module Block & 10 \\
\hline Mutation Rate (initial/final) & $0.25 / 0.05$ & Game Module Block & 10 \\
\hline Mutation Strength (initial/final) & $0.50 / 0.10$ & Decision Net Input Size & 150 \\
\hline Top Tier Selection & Above Avg & Decision Net Hidden Layer & 1 (75 nodes) \\
\hline
\end{tabular}

Table 4-1: ASHE 1.0 Parameter Settings

\subsubsection{Adaptation, Exploitation, and Reliability}

This subsection aims at answering three questions:

- How effective is ASHE in exploiting different opponent strategies?

- Can the evolutionary method in Section 4.2.2 evolve adaptive players with consistent performance?

- What are the specific adaptations that allow the players to exploit different opponents?

To answer these questions, the experiment introduced in the previous subsection was conducted 21 times. The champion in the last generation of each run was selected to play 500 games with each rule-based player. In addition, every rule-based player played against one of the cutting-edge game-theoretic players, Slumbot 2016 for 10000 games. All games were in the same format as specified in the previous subsection. Table 2 compares the average performance of the champions with the performance of Slumbot 2016. Performances are measured in $\mathrm{mBB} /$ hand, i.e. 1/1000 Big Blind per hand. 


\begin{tabular}{lll}
\hline Opponent & ASHE 1.0 & Slumbot \\
\hline Scared Limper & $998.6 \pm 2.619$ & $702.0 \pm 59.10$ \\
\hline Calling Machine & $40368 \pm 1989$ & $2761 \pm 354.6$ \\
\hline Hothead Maniac & $36158 \pm 1488$ & $4988.0 \pm 881.0$ \\
\hline Candid Statistician & $9800 \pm 1647$ & $4512.5 \pm 471.5$ \\
\hline
\end{tabular}

Table 4-2: Opponent Exploitation. ASHE 1.0 performs much better than Slumbot in exploiting highly exploitable opponent strategies $(\mathrm{mBB} /$ hand, confidence $=0.95)$.

Depending on the opponent strategy, the average performance of the champions is $42 \%$ to $1362 \%$ better than the performance of Slumbot 2016, making ASHE 1.0 a clear winner in the competition of opponent exploitation.

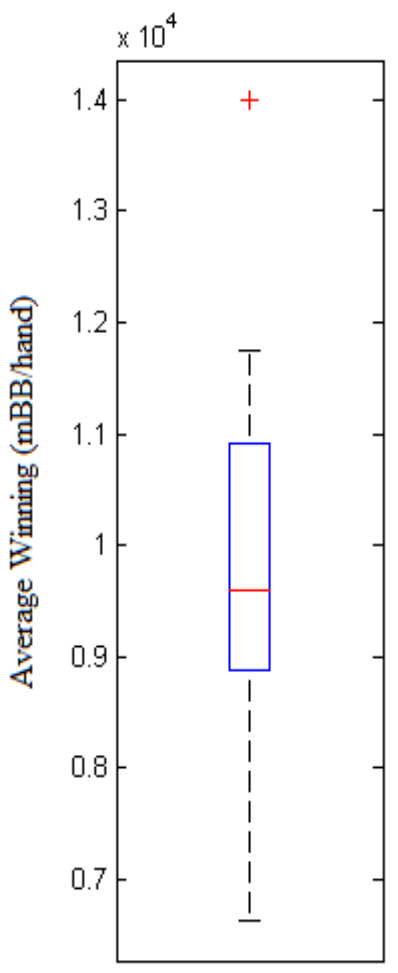

Candid Statistician

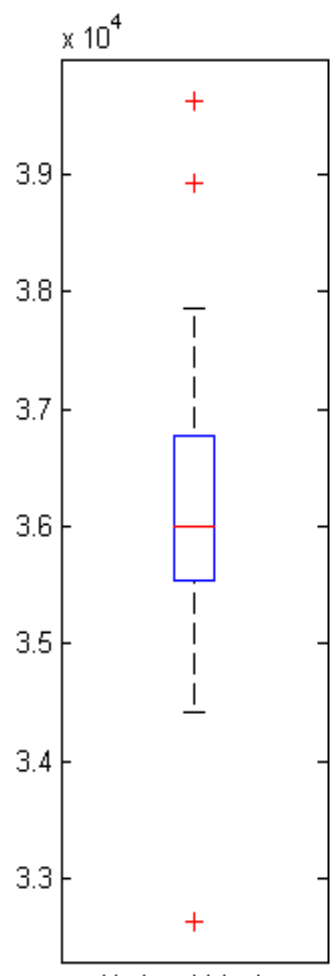

Hothead Maniac

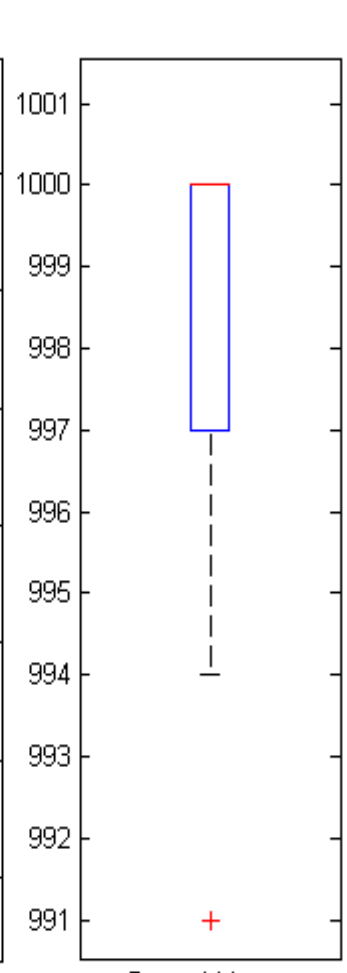

Scared Limper

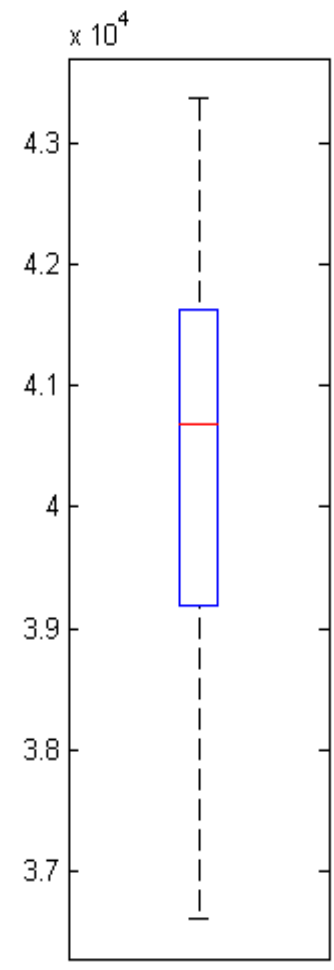

Calling Machine

Figure 4-4: Champion Performance vs. Rule-based Players. Last-generation champions evolved in 21 runs demonstrate consistent performance against rule-based opponents, and the evolutionary method is stable. 
Figure 4-4 shows boxplots of average winnings per hand of the 21 champions against each opponent. Samples outside 1.5 IQR (Inter-quartile Range) are marked as “+”. Champions from different runs demonstrate consistent performance, and the evolutionary method introduced in Section 3 is a reliable approach in evolving ASHE 1.0 agents with effective opponent exploitation.

The game logs of the champion with the highest fitness in all runs were analyzed to understand how adaptive LSTM players exploit each opponent. Table 4-3 shows some related statistics on the moves made by the champion.

Action frequencies are the probability of certain action being taken when they are available. Raise is available as long as a player has sufficient chips for the minimum raise. Fold is available only after opponent raises. The strategies of the Calling Machine and the Scared Limper make certain statistics unavailable (marked as “--"). The statistics in Table 3 as well as the game logs demonstrate many interesting adaptations for effective opponent exploitation.

\begin{tabular}{llllll}
\hline Stage & Attribute & CS & HM & CM & SL \\
\hline \multirow{2}{*}{ Preflop } & Avg. Raise (BB) & 2.59 & 3.59 & 3.80 & 2.07 \\
\cline { 2 - 5 } & Raise Frequency & $52.2 \%$ & $35.5 \%$ & $49.5 \%$ & $99.4 \%$ \\
\hline \multirow{2}{*}{ Flop } & Avg. Raise (BB) & 19.59 & 30.39 & 13.46 & - \\
\cline { 2 - 5 } & Raise Frequency & $30.6 \%$ & $22.7 \%$ & $48.4 \%$ & \\
\hline \multirow{2}{*}{ Turn } & Avg. Raise (BB) & 63.10 & 63.87 & 66.54 & \multirow{2}{*}{--} \\
\cline { 2 - 5 } & Raise Frequency & $26.8 \%$ & $11.8 \%$ & $34.8 \%$ & \\
\hline \multirow{2}{*}{ River } & Avg. Raise (BB) & 72.46 & 59.69 & 79.01 & \multirow{2}{*}{--} \\
& Raise Frequency & $10.3 \%$ & $11.5 \%$ & $14.0 \%$ & \\
\hline \multirow{2}{*}{ Game } & Fold Frequency & $24.9 \%$ & $4.9 \%$ & - & \multirow{2}{*}{--} \\
& Opponent Raise & 953 & 1938 & & \\
\hline
\end{tabular}

Table 4-3: Champion Action Statistics vs. Different Opponents. CS = Candid Statistician, $\mathrm{HM}=$ Hothead Maniac, $\mathrm{CM}=$ Calling Machine and $\mathrm{SL}=$ Scared Limper, Avg. Raises are measured in Big Blinds, i.e. $\mathrm{BB}$, and $\mathrm{BB}=100$. ASHE 1.0 adapts its action frequencies and bet/raise sizes against different opponents to exploit them effectively. 
First, the fold frequency of the champion when playing against the Candid Statistician is four times higher than the fold frequency against the Hothead Maniac. The champion adapts to the fact that the Candid Statistician's raises indicate strong hands, while the Hothead Maniac is inclined to bluff. Second, to exploit the strategy of the Scared Limper, the champion increases preflop raise frequency to nearly $100 \%$, forcing the extremely-conservative opponent to fold almost every hand. Third, when playing against the Hothead Maniac, the champion's raise frequency is much lower in all stages of the game. However, the average raises from the champion at the turn and the river are both close to $60 \mathrm{BBs}$. Note that a raise over $40 \mathrm{BBs}(\$ 4,000)$ generally leads to an all-in due to the Hothead Maniac's strategy and the size of the stack $(\$ 20,000)$. Thus, the champion exploits Hothead Maniac's strategy by folding weak hands, calling with fair hands, and inducing all-in with strong hands. Fourth, the champion's strategy against the Calling Machine features much higher raise frequency compared to its strategy against the Hothead Maniac. Game logs indicate that the champion is not bluffing. Rather, it simply recognizes the fact that the Calling Machine's moves are independent from the strength of its hands. Therefore, if the champion's hand is better than the average, it raises. In addition, the amount of a raise is strongly correlated with hand strength, which is different from the strategy against the Candid Statistician where the champion plays strong hands less aggressively and bluffs when the opponent appears passive.

Such adaptations enable ASHE 1.0 agents to make more profitable moves against different players, thus exploiting the weakness of opponent strategy effectively.

\subsubsection{Slumbot Challenge}

ASHE 1.0 agents are evolved through playing against rule-based players with only simple, weak strategies. Generally, genetic algorithms tend to achieve no fitness beyond 
the scope of evolution. Nevertheless, adaptive players have the potential to compete against previously unseen opponents through adaptation. How well can they generalize against new opponents with much stronger strategies?

To answer this question, 500 games were played between the champions from each run (as introduced in the previous subsection) and Slumbot 2016. The adaptive players lost to Slumbot 2016 by an average of $\underline{426 \mathrm{mBB} / \mathrm{hand}}$.

Game logs show that ASHE 1.0 adopts an aggressive strategy that is essentially a biased combination of the strategies against the rule-based players, and is able to bluff Slumbot 2016 effectively when holding relatively weak hands. However, Slumbot 2016 performs much better when holding strong hands. In particular, Slumbot 2016 uses value bets (i.e. small to mid-sized bets aimed at inducing a call or re-raise) effectively. In contrast, ASHE 1.0 tends to raise rather aggressively with a strong hand, which usually reveals true hand strength and leads to a fold in response.

This problem may be caused by the fact that ASHE 1.0 agents have rarely seen value bets from the rule-based opponents; neither is value bet necessary for exploiting them. Two out of the four rule-based players almost never fold to any raise. Furthermore, the Candid Statistician can be quite reckless in calling or raising when the pot is small.

To alleviate this problem, two adjustments were made to the decision algorithm: (1) raise amount was restricted to be integer times of a half of the pot size, up to twice the pot size and all-in, and (2) if the desired raise amount was smaller than a fourth of the pot size, the player was made to call or check instead of raise. These rule-based adjustments limit raise amount to five options, making each option representing a much wider range of hands, which helps conceal true hand strength. After the adjustments, the agents' performance improved considerably, losing $115 \mathrm{mBB} /$ hand, or approximately $1 / 10$ of a big blind per hand against Slumbot 2016. 
These results suggest that ASHE 1.0 agents can adapt to opponents that are not seen in training by combining strategies against the training opponents. However, when the opponent is significantly stronger than the training opponents, such as Slumbot 2016, it still loses in heads-up matches against it.

\subsection{DisCUSSION AND CONCLUSION}

ASHE 1.0 is the first attempt in evolving an adaptive recurrent-neural-networkbased computer agent for HUNL, which is considered one of the most challenging imperfect information games. The system lays a solid foundation for this line of research and provides valuable insights into the methodology.

First, the experimental results showed that LSTM neural networks can be leveraged to represent game states and model opponent strategies. The ASHE 1.0 architecture enables the agents to adapt their behaviors, thereby exploiting different opponents. In particular, when facing opponents with highly exploitable strategies, the adaptive agents are significantly more effective than top-ranking equilibrium-based agents. Second, ASHE 1.0 presents an algorithmic framework for evolving adaptive RNN-based agents for HUNL and other imperfect information games. Such evolutionary methods do not require labeled training data and are therefore applicable to a wider range of problems. The TSER method preserves advantageous genotypes while creating high-quality offspring, and is particularly effective in evolving computer agents for games of chance. Third, the experimental results show that the evolutionary method is reliable, i.e. the agents generated from different runs demonstrate consistent performance. Fourth, ASHE 1.0 can adapt to opponents that are not seen during training by combining strategies learned through evolution. However, if the opponents are much stronger than the training opponents, it still loses in a heads-up match. 
Thus, the empirical results and analysis of ASHE 1.0 validate the methodology. They also point out potential directions for further improvement. From an architectural perspective, ideally, the LSTM module, and in particular, the opponent module should contain enough blocks to extract and encode all useful features. This design is especially important when playing against more sophisticated opponents, as exploitable patterns in their strategies tend to be conditional and well-hidden. On the other hand, evolving bigger networks is generally less efficient. Hence, the first challenge is to develop an architecture that uses (relatively) small LSTM modules to extract and encode sufficient features in opponent strategies.

In addition, ASHE 1.0 models the opponents implicitly. It does not predict the opponent's actions or estimate the opponent's (expected) hand strength directly. In fact, all information on the game states of the hand in play and the opponent's strategy is represented by the aggression score, i.e. the output of the decision network. Implicit opponent modeling makes it difficult to estimate the expected value for possible actions. Hence, ASHE 1.0 adopts an intuitive rule-based approach to select the next action based on the aggression score. Such intuitive methods may be effective against opponents with relatively simple strategies. However, their performance against strong opponents, e.g. Slumbot 2016, is unsatisfactory.

Therefore, the second direction is explicit opponent modeling, i.e. to predict hand strength, opponent actions, etc. The prediction can be used to estimate the expected values for possible actions, thus allowing the agents to make more accurate decisions. The next chapter introduces ASHE 2.0, an upgrade of the system that addresses both of these problems. 


\section{Chapter 5: Pattern Recognition Tree and Explicit Modeling}

This chapter introduces ASHE 2.0, which establishes an effective and extensible architectural framework for building LSTM-based adaptive agents for HUNL and other imperfect information games.

Compared to the previous version, ASHE 2.0 makes two improvements. First, it introduces the Pattern Recognition Tree (PRT), which enhances the ability of modeling complex opponent strategies while keeping the LSTM modules small enough for efficient evolution. Second, it models the opponents explicitly, thus allowing the agents to make decisions by estimating the expected utility of each available action.

Experimental results show that ASHE 2.0 models and exploits both weak and strong opponents effectively. In particular, it achieves significantly better performance in matches against top-ranking equilibrium opponents than the previous version and tied statistically with Slumbot 2017, an upgraded and much stronger version of Slumbot 2016, which was used in the ASHE 1.0 experiments.

This chapter consists of four sections. Section 5.1 introduces the architecture of ASHE 2.0. Section 5.2 introduces the genetic algorithm to evolve the agents. Section 5.3 presents and analyzes the experimental results. Section 5.4 summarizes the findings and discusses directions for further improvements.

\subsection{ASHE 2.0 ARCHITECTURE}

The ASHE 2.0 architecture is an RNN-based architectural framework that enables the agents to capture more subtle patterns in opponent strategies through explicit opponent modeling. The architecture allows efficient training by the evolutionary methods specified in Chapter 4, and is effective against opponents that are much stronger than opponents seen during evolution. 
Subsection 5.1.1 presents the overall architecture and introduces the motivation of the design. Subsection 5.1.2 and subsection 5.1.3 focuses on the components, e.g. the opponent model and the decision algorithm, respectively.

\subsubsection{Motivation and Framework}

The ASHE 2.0 architecture builds on ASHE 1.0 by expanding the opponent model. Instead of the LSTM modules only, it now contains the Pattern Recognition Tree (PRT) and two LSTM estimators. The PRT and the LSTM estimators extract exploitable patterns, predicts opponent moves, and estimate showdown value based on opponent hand strength. In addition, ASHE 2.0 decides its moves based on action utilities estimation instead of the aggression score as in ASHE 1.0. The decision algorithm evaluates the expected utility of each available action using the outputs of the opponent model, and selects the action with the highest utility (Figure 5-1).

\section{Motivation: Pattern Recognition Tree}

As is presented in Chapter 4, ASHE 1.0 achieved remarkable performance against highly exploitable opponents through implicit opponent modeling and adaptation. However, it was less effective playing against Nash-equilibrium-based opponents whose strategy is considerably less exploitable. This result is attributable to the fact that the exploitable patterns of strong opponents are usually conditional and difficult to discover. For instance, Slumbot 2016 (the version in ASHE 1.0 experiments) tends to underestimate its hand value when facing a paired board. Thus, bluffing on paired-flops is a profitable move against this agent in the long run. As another example, when holding a two-card flush draw on the flop, Slumbot 2016 semi-bluffs noticeably more often than most players. Since

a flush draw hits only (roughly) $1 / 3$ of the time, this pattern can be exploited by continuing 
with a reasonably wider range of hands under corresponding conditions. Thus, the ability to model complex patterns in opponent strategy is critical when facing strong opponents.

In ASHE, opponent strategies are modeled through recurrent neural networks (LSTM modules). Generally, larger networks are more effective in modeling complex strategies. On the other hand, bigger modules require longer training and may render evolution painfully slow.

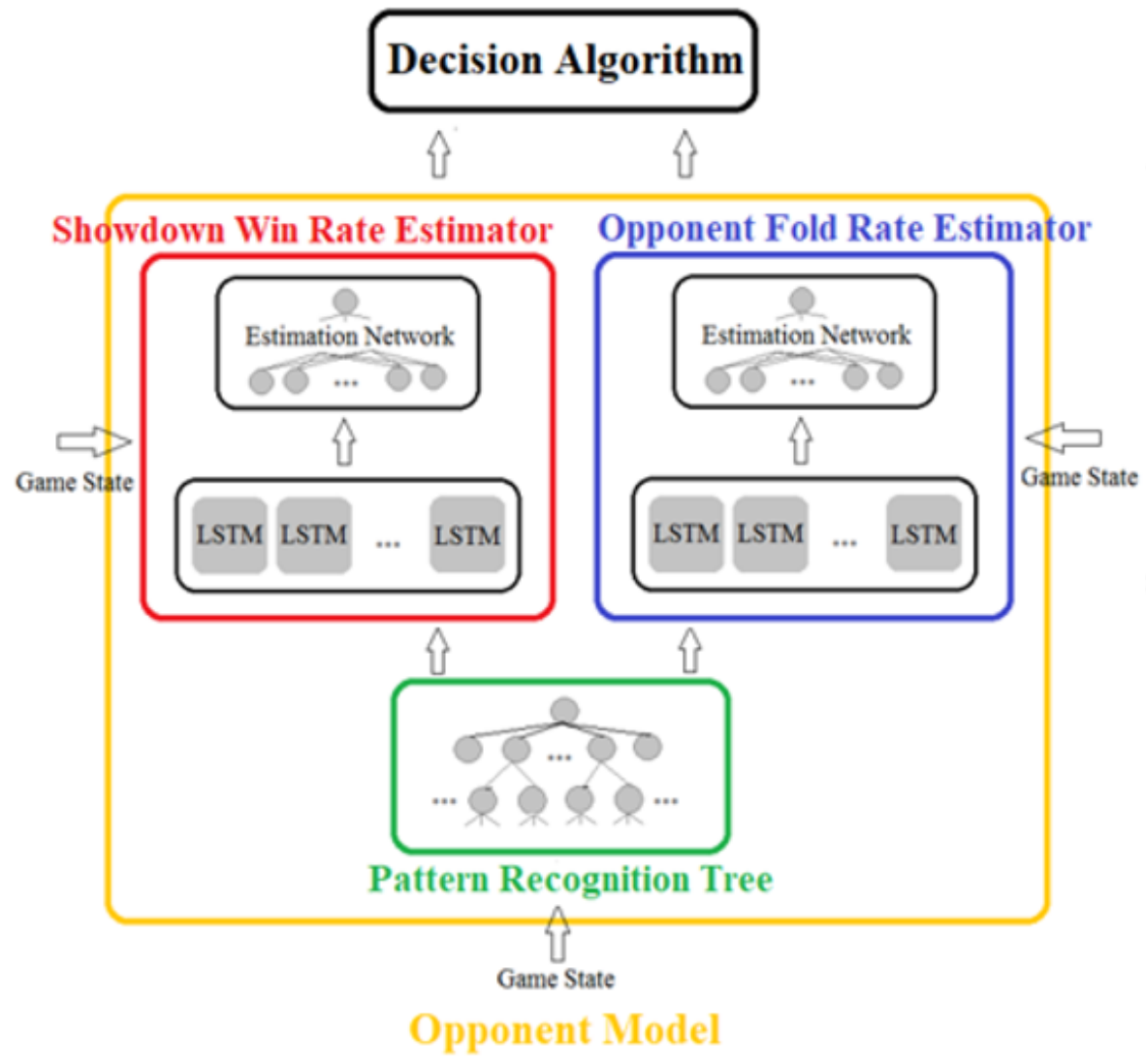

Figure 5-1: ASHE 2.0 Architecture. ASHE 2.0 consists of two parts: a decision algorithm and an opponent model. The opponent model contains three components: the Showdown Win Rate Estimator, the Opponent Fold Rate Estimator, and the Pattern Recognition Tree (PRT). The PRT and LSTM estimators allow ASHE 2.0 to model the opponents explicitly, thus making decisions on a more accurate basis (i.e. expected action utilities). 
The PRT is introduced to address this dilemma. It is a tree structure whose nodes correspond to the game states and collect useful statistical data for each state. The data is sent to the LSTM estimators as part of the inputs when corresponding game state occurs. Thus, the PRT is essentially a feature extractor, which extracts useful statistical features from different game states and organizes them accordingly.

The PRT serves two purposes. First, it provides useful features at the right time, i.e. at the corresponding game state. Second, it alleviates the burden of extracting long-term patterns in opponent strategies for the LSTM modules. Thus, when combined with the LSTM modules, the PRT allows the opponent model to capture complex exploitable patterns while keeping the network modules relatively small. Since the PRT itself does not need optimization through evolution, it can make training/evolution more efficient.

\section{Motivation: Explicit Modeling and Utility-based Decision-making}

Another limitation of ASHE 1.0 is implicit opponent modeling, i.e. all patterns are encoded by the output of the decision network, and the agents do not predict opponent moves or estimate opponent hand strength explicitly. Therefore, it is impossible to compute the utility of available actions. Hence, decisions have to be made based on intuitive rules that are unreliable. Moreover, advanced tactics such as slow-play and check-raise require estimation of utility and/or prediction of opponent actions.

For example, in order to justify slow-playing a strong hand (i.e. checking/calling instead of betting/raising with a strong hand in order to conceal hand strength), opponent should be likely to act aggressively facing a check/call and fold to a bet. Similarly, semibluffs are only profitable if the opponent is likely to fold under pressure, or the expected utility for a bet/raise is higher than simply check-folding the hand. 
Hence, ASHE 2.0 models the opponent explicitly using the PRT and the LSTM estimators. The PRT maintains a set of statistics for each sequence of actions (game state) seen in games against an opponent, collecting information on the opponent's strategy from every hand. Both estimators receive input features extracted from the current game state and the PRT. The Showdown Win Rate Estimator evaluates the showdown value, i.e. the probability of ASHE holding a hand better than the opponent. The Opponent Fold Rate Estimator estimates the probability of the opponent folding to a bet/raise. The estimations are sent to the decision algorithm, which evaluates the expected utility for each possible action and selects the action with the highest utility. Compared to the previous version, ASHE 2.0 agents thus make decisions on a more reliable basis and demonstrate more advanced tactics in their games.

The ASHE 2.0 architecture provides a structural framework for evolving LSTMbased adaptive agents for imperfect information games. The PRT allows smaller neural network modules and more efficient evolution. The LSTM estimators in the opponent model predict opponent actions and estimate showdown values. The decision algorithm makes decisions based on expected utility.

While the ASHE 2.0 architecture was originally designed for HUNL, the framework and principles are not limited to poker. PRTs can be constructed for any statebased imperfect information game to reduce network module complexity and improve training efficiency. Similarly, LSTM estimators can be evolved to predict the probability of opponent actions in other games, and the decision algorithm adjusted in the context of the games. The next two subsections details these components of the system. 


\subsubsection{Opponent Model}

The opponent model predicts opponent actions and estimates showdown values, providing the decision algorithm with necessary information to evaluate action utilities. It contains the PRT and two LSTM estimators, i.e. the Opponent Fold Rate Estimator, and the Showdown Win Rate Estimator.

\section{Pattern Recognition Tree}

The PRT collects statistical data on opponent strategy for each game state. The data is accumulated from all hands played against the current opponent and stored in tree nodes corresponding to the game states. The data is used to derive input features for the LSTM estimators at different game states. In ASHE 2.0, the "game states" in the PRT refer to action sequences, e.g. preflop on the button: bet half the pot (agent), fold (opponent). They do not include information on community cards or private cards. Features derived from the cards are sent to the LSTM estimators directly.

Figure 5-2 illustrates the structure of the PRT. The root of the tree represents an initial state of the game: it can be either preflop as the button or preflop as the Big Blind, with no action taken by either player. Thus, in a typical HUNL match, two PRTs are created, each corresponding to one of the two positions (i.e. the Button or the Big Blind). Leaf nodes represent terminal states of the game. They can be either a showdown or a fold from one of the players. Each non-leaf node corresponds to a game state, i.e. a sequence of actions from both players. The non-leaf nodes also represent decision points where either the agent or its opponent must make the next move. 


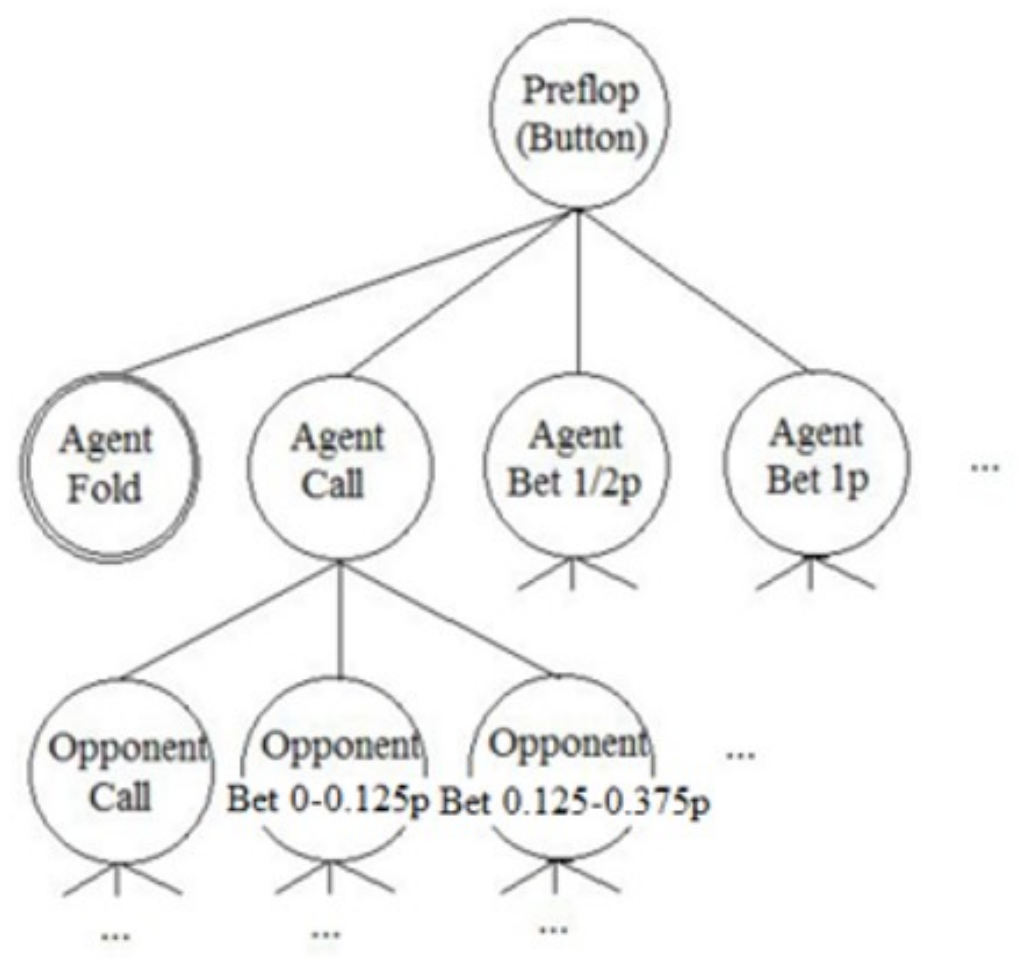

Figure 5-2: A Pattern Recognition Tree. The PRT collects statistical data on opponent strategy for each game state, stores the data in corresponding nodes, and uses the data to derive input features for the LSTM modules. Thus, the PRT provides useful features to the opponent model and keeps the LSTM modules small enough for efficient evolution.

Each non-leaf PRT node stores a set of statistical data called the node stats. The attributes of the data are:

- State Frequency $(f)$ : the number of times the game state represented by the node occurring in all hands played against the current opponent.

- Showdown Counts $\left(\mathrm{c}_{\mathrm{sd}}\right)$ : the number of showdowns after reaching the game state represented by the node.

- Fold Counts $\left(\mathrm{c}_{\mathrm{of}}\right)$ : the number of times the opponent eventually folding its hand after reaching the game state represented by the node. 
- Showdown Strength $(\bar{s})$ : the average opponent hand strength in all showdowns after passing the game state represented by the node.

The node stats capture patterns in the opponent strategy at different game states. For instance, if the opponent is over-cautious when facing consecutive bets at both the flop and the turn, the $c_{\text {of }}$ will be high for every node along that path, suggesting an opportunity for bluffing. At the beginning of a match, two PRTs corresponding to each position (i.e. the button and the Big Blind) are initialized with only a root node. Nodes are inserted to the trees when their corresponding game states occur for the first time in the match.

Suppose in the first hand of a game session, ASHE plays the button and decides to call the Big Blind preflop, a node representing that action will be added as a child of the root. At this point, all attributes in the node stats are set to null and cannot be used for deriving input features to the LSTM estimators. Instead, a set of default inputs are sent to the estimators. Suppose the opponent decides to raise by two Big Blinds, a node representing the raise will be added to the PRT as a child of the node that represents ASHE's call. This process continues until the current hand is finished.

After each hand, node stats in non-leaf nodes along the path (from the root to the leaf) will be updated based on the result of the game. State frequencies of the nodes will be increased by one. If the opponent folds in the end, fold counts of the nodes will be increased by one. If a showdown occurs, showdown counts of the nodes will be increased by one, and showdown strengths updated based on the opponent's hole cards. The hand strength of the opponent is defined as the probability of the opponent's hand beating a random hand.

Suppose that in another hand, ASHE plays the button and decides to call the Big Blind preflop once again. This time, a node representing that state already exists; hence, it 
is unnecessary to create a new node, and the existing node will be visited. If the state frequency is greater than a threshold, i.e. the node has been visited enough times to collect meaningful data, the node stats will be used to derive input features for the LSTM estimators; otherwise, the default inputs will be used. As the match proceeds, the PRTs grow, and the node stats become increasingly reliable, especially for game states that occur frequently.

Since each node in the PRT corresponds to a game state, an important trade-off lies in the definition of the game states. The number of distinct game states should not be too large, otherwise most of the nodes cannot be visited often enough in a game session, and the node stats become useless. On the other hand, if the number of game states in the PRT is too small, the PRTs cannot distinguish enough game states and capture complex patterns in opponent strategies.

The raw game states in HUNL contain the actions, the community cards, and the private cards, making the total number of states huge. Thus, these states must be merged into more general states in the PRT. To control the size of the trees and to ensure that most nodes are visited frequently enough to provide meaningful node stats, PRTs in ASHE 2.0 contain nodes for each action sequence and do not distinguish game states by community or private cards.

All bets/raises are scaled with respect to the size of the pot to make the PRTs generalizable to games of different stakes. ASHE's bets are restricted to 0.5, 1.0, 1.5, 2.0 pot size, and all-in. The opponent's bets are discretized into seven buckets: $(0,0.125)$, $(0.125,0.375),(0.375,0.75),(0.75,1.25),(1.25,2.0),(2.0,4.0),(>4.0)$, also with respect to the size of the pot. 
Thus, the PRT collects and organizes statistical data on opponent strategies. The input features derived from node stats capture exploitable patterns, allowing the LSTM modules to be smaller and training more efficient.

\section{LSTM Estimators}

The LSTM estimators provide essential information for the decision algorithm to evaluate the utility of each possible action. The Showdown Win Rate Estimator (SWRE) estimates the probability of ASHE's hand beating its opponent's if a showdown occurs. The Opponent Fold Rate Estimator (OFRE) estimates the probability of the opponent folding its hand if ASHE bets or raises. Both the SWRE and the OFRE share the same LSTM-based neural network architecture, illustrated by Figure 5-3.

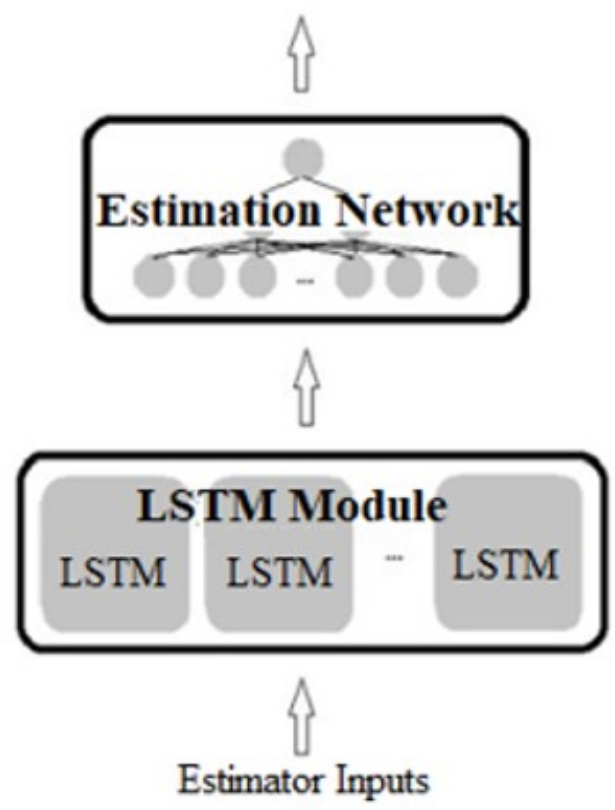

Figure 5-3: An LSTM Estimator. Both LSTM estimators, i.e. the SWRE and the OFRE, contain an LSTM module and an estimation network. The estimators receive inputs derived from the raw game state and the node stats in the PRTs. They predict opponent actions and evaluate showdown values, which are used by the decision algorithm to estimate action utilities. 
In ASHE 2.0, each LSTM module contains 50 LSTM blocks as described in Subsection 4.1.1. Each block contains ten cells. These LSTM blocks are organized into a single layer. The estimation network is a fully connected feed-forward neural network with one hidden layer.

Table 1 defines the input features. The first four input features are derived from node stats in the PRTs, which provides statistical information on the opponent's strategy given the same action sequence in the past. The rest of the features provide information on the current state of the game. Notations of node stats are the same as defined in the previous subsection. S and F refers to the SWRE and the OFRE, respectively. These input features collectively not only encode the current game state but also reflect the history of previous games against the opponent.

\begin{tabular}{lll}
\hline \hline Feature Name & Definition & Input \\
\hline Normalized Frequency & $\sigma(f / 10)$, where $\sigma$ is the sigmoid function. & $\mathrm{S} \& \mathrm{~F}$ \\
\hline Fold Rate & $\left(c_{\mathrm{of}}+1\right) /(f+3)$, smoothed by $1 / 3$. & $\mathrm{S} \& \mathrm{~F}$ \\
\hline Showdown Rate & $\left(c_{\mathrm{sd}}+1\right) /(f+3)$, smoothed by $1 / 3$. & $\mathrm{S}$ \\
\hline Expected Strength & $\bar{s}$ if $c_{\mathrm{sd}}>5,\left(0.85 \cdot\left(5-c_{\mathrm{sd}}\right)+\bar{s} c_{\mathrm{sd}}\right) / 5$, otherwise. & $\mathrm{S}$ \\
\hline Flush and Straight Draw & $\begin{array}{l}\text { Probability of a random hand hitting flush or straight } \\
\text { given the board. }\end{array}$ & $\mathrm{F}$ \\
\hline Pair(s) & $\begin{array}{l}\text { 0: no pair on board, 0.5: one pair on board, 1.0: two } \\
\text { pairs on board. }\end{array}$ & $\mathrm{F}$ \\
\hline Betting Round & $\begin{array}{l}\text { Binary indicators of the four rounds, i.e. preflop, flop, } \\
\text { turn, and river. }\end{array}$ & $\mathrm{S} \mathrm{\&} \mathrm{\textrm {F }}$ \\
\hline Raw Hand Strength & $\begin{array}{l}\text { Probability of ASHE's hand beating a random hand given } \\
\text { the board. }\end{array}$ & $\mathrm{S}$ \\
\hline Opponent Total Bet & Opponent's total bet normalized by the initial stack size. & $\mathrm{S} \& \mathrm{~F}$ \\
\hline ASHE Total Bet & ASHE's total bet normalized by the initial stack size. & $\mathrm{S} \mathrm{\&} \mathrm{F}$ \\
\hline \hline
\end{tabular}

Table 5-1: Estimator Inputs. The two LSTM estimators in ASHE 2.0 take inputs derived from both the raw game states and the PRT node stats to predict opponent actions and estimate showdown values. 
At the beginning of each hand, all LSTM blocks in the estimators are set to their initial states. Suppose ASHE plays the button and needs to decide its first action preflop. The features in Table 5-1 will be derived from the raw game state and the node stats in the current PRT node (in this case, the root of the tree). After the input features are sent to the LSTM modules in both estimators, the states of the LSTM modules will be saved as the current states. Note that if the node does not exist or the node stats are not reliable, the default inputs will be used instead.

The output of the SWRE based on the current states will be sent to the decision algorithm to estimate the utility for every possible action, including fold, check/call, and bet/raise. ASHE assumes that the opponent will never fold when check is available. Therefore, the probability of the opponent folding its hand after a check or call is always assumed to be zero. However, for a bet/raise, the probability of inducing a fold from the opponent, a.k.a. the fold equity, is crucial for estimating the utility.

To compute the fold equity, the system need to look one step ahead by assuming that ASHE makes a bet/raise of a certain size. Input features will be computed using the node stats that correspond to the action. After receiving the input features and predicting the fold equity for the bet/raise, the OFRE will be reset to the current states. Fold equity prediction will be made for bets/raises of all legitimate sizes.

As the game proceeds, input features derived from the raw game states and the node stats corresponding to each action will be sent to the LSTM estimators. Thus, the estimators can make predictions based on every move in this hand as well as the patterns that are captured in previous games with similar situation. 


\subsubsection{Decision Algorithm}

The decision algorithm computes the utility of available actions using the outputs from the LSTM estimators and selects the action with the highest utility as the next move. Figure 5-4 outlines this algorithm. Parameters $b_{\text {opp }}$ and $b_{A S H E}$ refer to total chips committed in the pot by the opponent and ASHE, respectively.

When estimating the expected utility of an action, the decision algorithm makes two assumptions. First, it assumes that the estimations from the LSTM estimators are the true values. Second, it assumes that if the opponent does not fold to a raise, it will call, and the game will go to a showdown with no more chips committed to the pot. Although neither of these assumptions always hold in reality, experimental results indicate that the utility estimations based on the decision algorithm is a reasonable approximation.

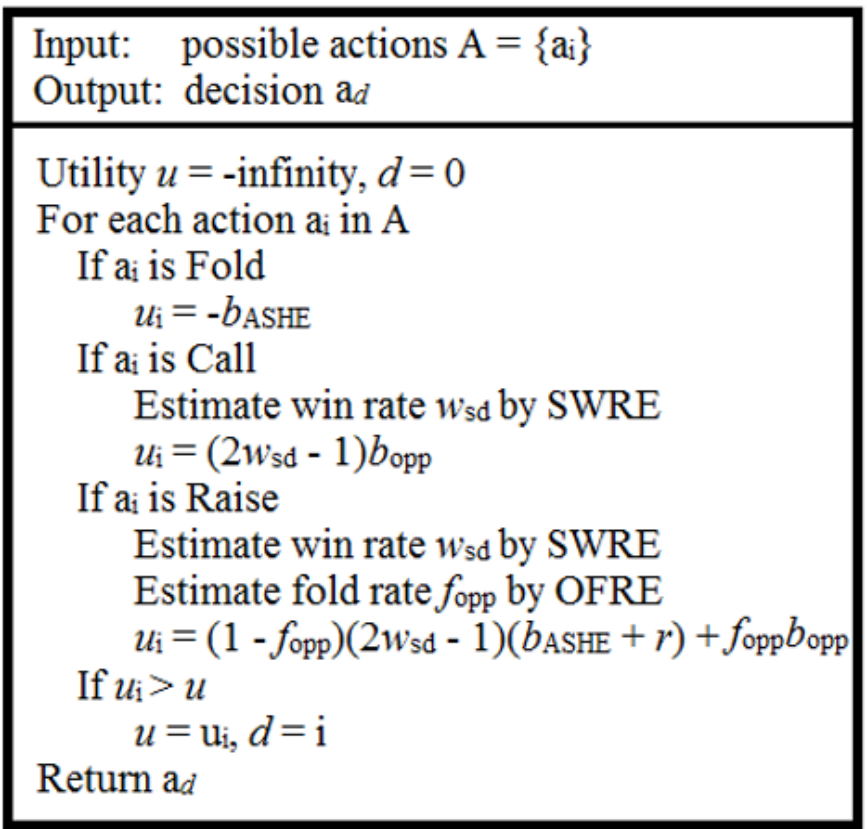

Figure 5-4: Decision Algorithm of ASHE 2.0. The algorithm estimates the expected utilities of each available action and selects the best move. The estimations are based on the outputs of the LSTM estimators. Compared to the decision algorithm in ASHE 1.0, this algorithm makes decision on a more accurate and quantitative basis. 
In addition, a restriction is placed on ASHE's actions: when facing a four-bet (as the Big Blind) or a three-bet (as the Button) ASHE can only respond with a fold, a call, or an all-in. This restriction simplifies utility estimation and reduces the size of the PRTs.

In sum, the ASHE 2.0 architecture uses the PRTs to collect statistical information on the opponent's moves given different at decision points. The LSTM estimators consider such information and the game state at each decision point in the current hand to estimate ASHE's winning probability in a showdown and the probability of the opponent folding to bets/raises. The decision algorithm uses these outputs to approximate the expected utility of available actions and selects the best move accordingly.

Evolving high-performance computer agents for large-scale imperfect information games is a challenging task. The ASHE 2.0 architecture provides a structural framework for building such agents and should be applicable to domains other than poker as well.

\subsection{EVOLUTION OF THE LSTM ESTIMATORS}

The performance of ASHE depends on the accuracy of the LSTM estimators. Although it is clear what the LSTM estimators need to do, how can they be trained to do it? As is discussed in Subsection 4.2.1, it is difficult to obtain sufficient labeled data for training adaptive poker agents. In fact, even the definition of "correctness" is unclear. For example, what is the chance of the opponent folding to a half-pot raise given the current game state and the history of previous games? While most professional human players may be able to figure out a reasonable range, it is impossible to give an answer that is correct in an absolute sense.

Therefore, the LSTM estimators cannot be trained through supervised methods. A major technical insight in this work is that it is possible to evolve the LSTM estimators for 
their tasks with genetic algorithms as outlined in Section 4.2. This approach makes the method applicable to problems with little or no labeled training data.

In the genetic algorithm, a population of agents are created with randomly initialized LSTM estimators at first. They are evaluated based on their fitness, i.e. their performance against a variety of training opponents. These opponents require different counter-strategies for effective exploitation. Thus, the agents with adaptive behaviors that can exploit various strategies are rewarded by higher fitness. The agents with the highest fitness survive and reproduce via mutation and crossover. The others are replaced by the offspring. Selection and reproduction are done iteratively, thus improving the overall fitness of the population. Note that the agents do not have to know what the accurate estimations are or what actions are the best; it is sufficient to select agents that perform relatively well in the population.

Specifically, during evolution, agents are represented by theirs numeric genomes, which are constructed by concatenating all parameters in the estimators, i.e. the weights in the LSTM module, the initial states of all LSTM blocks, and the weights of the estimation network.

To preserve advantageous genotypes against occasional poor luck, Tiered Survival and Elite Reproduction (TSER, as introduced in Section 4.2.2) is adopted. Survivors are divided into two tiers based on their fitness. Agents in the top tier (i.e. the elite tier), can reproduce and do not mutate. Agents of the second tier mutate but cannot reproduce. Thus, genotypes from more agents can be preserved without compromising the performance of the offspring.

Evolving agents with multiple modules can be inefficient, especially at an early stage. Advantageous genotypes of one module may be coupled with poor genotypes of another, causing low performance and loss of progress. Therefore, evolution is organized 
into a series of sessions, with each session dedicated to evolving one of the estimators. When a new session begins, the champion of the previous session is duplicated to form the agent population. The weights in the estimator to be evolved are mutated, and the weights in the other estimator frozen. Thus, the genetic algorithm is able to discover effective LSTM estimators efficiently, although there is no data for supervised training.

\subsection{EXPERIMENTAL RESULTS}

ASHE 2.0 agents were evolved by playing against opponents with different level of exploitability. A total of 11 agents were used to evaluate ASHE's performance against both weak and strong opponents.

Below, Subsection 5.3.1 provides details on the experimental setup and match formats, Subsection 5.3.2 introduces the opponents in training and testing, and Subsection 5.3.3 presents and discusses the results.

\subsubsection{Experimental Setup}

Table 5-2 presents the parameters in the experiments. During training, evolution was organized into sessions. Each session evolves one of the two LSTM modules, i.e. the OFRE or the SWRE, and freezes the other (Subsection 5.2). The champions of the last generation were used for evaluation.

Mutation followed Gaussian distribution. As evolution proceeded, mutation rate and mutation strength (i.e. the standard deviation of the Gaussian distribution) descended linearly. Similar to the ASHE 1.0 experiments, fitness was measured by the Average Normalized Earnings (ANE, as in Subsection 4.3.3). Survivors whose fitness was higher than the mean of fitness among all survivors became the elites in TSER. 


\begin{tabular}{llll}
\hline Parameter & Value & Parameter & Value \\
\hline Number of Generation & 500 & LSTM Block Size & 10 \\
\hline Generation(s) per Session & 50 & Blocks per Module & 50 \\
\hline Population Size & 50 & Estimation Net Input Size & 500 \\
\hline Survival Rate & 0.30 & Estimation Net Hidden Layer & 1 \\
\hline Mutation Rate (initial/final) & $0.25 / 0.05$ & Estimation Net Hidden Nodes & 50 \\
\hline Mutation Strength (initial/final) & $0.50 / 0.10$ & Hands/Match & 1000 \\
\hline Elite Selection & Above Avg & Duplication & Yes \\
\hline
\end{tabular}

Table 5-2: ASHE 2.0 Parameters. These settings should be considered as a reference. Reasonably different parameter settings do not affect the performance noticeably.

In each generation, the agents played two matches against every opponent. Card decks in the first match were duplicated and played again in the second match. The players switched seats between the two matches, and the PRTs of the agents were reset before entering the second match. At the beginning of each hand, the chip stacks of the two players were reset to the original stack size, $\$ 20,000$. The stakes were $\$ 50 / \$ 100$ with no ante. The game format was consistent with HUNL matches in the Annual Computer Poker Competition Protocol.

\subsubsection{Training and Testing Opponents}

Table 5-3 describes the agents used for training and testing the agents. HM, CM, SL and CS are four agents with highly exploitable strategies that require different (and in some cases, opposite) counterstrategies. LA, LP, TP, and TA are less exploitable versions of HM, CM, SL, and CS, respectively.

RG, HP, and SB were used only for testing. RG is highly exploitable, but its strategy is dynamic. HP is a strong agent based on common tactics and strategies adopted by professional players. Slumbot 2017 was the best Nash-equilibrium-based agent that was publicly available at the time of the experiments. It was an upgrade of Slumbot 2016, which was used in the ASHE 1.0 experiments and is considerably less exploitable. 


\begin{tabular}{lll}
\hline Name (Code) & Exploitability & Description \\
\hline Hothead Maniac (HM) & Very High & Always raises by half or one pot randomly. \\
\hline Calling Machine (CM) & Very High & Always checks or calls. \\
\hline Scared Limper (SL) & High & Calls when holding the nut; else, folds to any bet. \\
\hline Candid Statistician (CS) & High & $\begin{array}{l}\text { Bets 1/4 to one pot depending on raw hand strength, } \\
\text { checks/calls with marginal hands, folds weak hands. }\end{array}$ \\
\hline Random Gambler (RG) & High & $\begin{array}{l}\text { Randomly switching from other highly exploitable } \\
\text { strategies every 50 hands. }\end{array}$ \\
\hline Loose Aggressive (LA) & High & Bets/raises aggressively with a wide range of hands. \\
\hline Loose Passive (LP) & High & Calls with most hands, folds weak hands, rarely raises. \\
\hline Tight Passive (TP) & High & Calls with good hands, folds most hands, rarely raises. \\
\hline Tight Aggressive (TA) & Moderate & Similar to CS, with refined hand ranges and bluffing. \\
\hline Half-a-Pro (HP) & Low & $\begin{array}{l}\text { TA with advanced strategies such as floating, check- } \\
\text { raising, and multi-shot bluffing. }\end{array}$ \\
\hline Slumbot 2017 (SB) & Very Low & $\begin{array}{l}\text { Updated version of the runner-up in ACPC 2016, a } \\
\text { cutting-edge equilibrium-based poker agent. }\end{array}$ \\
\hline
\end{tabular}

Table 5-3: Training and Testing Opponents. These 11 agents form a pool of training and testing opponents with different weakness types and exploitability levels.

\subsubsection{Results and Analysis}

To investigate training opponents' influence on the performance of the agents, four instances of ASHE 2.0 were evolved with different evolution settings.

Agent A1 was evolved by playing against a group of highly exploitable opponents with diversified strategies, i.e. HM, CM, SL and CS. Agent A2 was evolved by playing against less exploitable opponents with similarly diversified weaknesses, i.e. LA, LP, TP, and TA. Agent A3 was evolved against two pairs of opposite strategies (i.e. maniac and passive) with different level of exploitation, HM, SL (relatively high exploitability), and LA, TP (relatively low exploitability). Agent A4 was evolved against HM, CM, SL and CS (the same as A1) for the first 200 generations, and LA, LP, TP, and TA (the same as A2) for the other 300 generations. 


\begin{tabular}{lllllll}
\hline & HM & CM & SL & CS & RG & HP \\
\hline $\mathrm{A}_{1}$ & $44241 \pm 6111$ & $48361 \pm 5012$ & $999 \pm 1.2$ & $9514 \pm 1515$ & $6992 \pm 600$ & $37 \pm 56$ \\
\hline $\mathrm{A}_{2}$ & $40010 \pm 5912$ & $45703 \pm 4998$ & $998 \pm 1.5$ & $8071 \pm 1390$ & $8422 \pm 709$ & $225 \pm 62$ \\
\hline $\mathrm{A}_{3}$ & $47086 \pm 6539$ & $22133 \pm 2407$ & $999 \pm 0.3$ & $1954 \pm 286$ & $5699 \pm 545$ & $-331 \pm 73$ \\
\hline $\mathrm{A}_{4}$ & $42333 \pm 6037$ & $46114 \pm 4985$ & $999 \pm 1.4$ & $9116 \pm 1403$ & $8996 \pm 721$ & $278 \pm 59$ \\
\hline $\mathrm{SB}$ & $4988 \pm 881$ & $2761 \pm 355$ & $702 \pm 59$ & $4512 \pm 472$ & $2102 \pm 393$ & $152 \pm 50$ \\
\hline
\end{tabular}

\begin{tabular}{llllll}
\hline & LA & LP & TP & TA & SB \\
\hline $\mathrm{A}_{1}$ & $13506 \pm 988$ & $9060 \pm 598$ & $1252 \pm 70$ & $107 \pm 54$ & $-59 \pm 77$ \\
\hline $\mathrm{A}_{2}$ & $18241 \pm 975$ & $12444 \pm 670$ & $1412 \pm 81$ & $431 \pm 90$ & $-8 \pm 55$ \\
\hline $\mathrm{A}_{3}$ & $23074 \pm 1021$ & $4781 \pm 523$ & $1493 \pm 82$ & $-106 \pm 65$ & $-512 \pm 66$ \\
\hline $\mathrm{A}_{4}$ & $20005 \pm 1006$ & $15372 \pm 634$ & $1488 \pm 91$ & $509 \pm 88$ & $5 \pm 61$ \\
\hline $\mathrm{SB}$ & $2449 \pm 460$ & $623 \pm 41$ & $603 \pm 51$ & $284 \pm 49$ & - \\
\hline
\end{tabular}

Table 5-4: ASHE 2.0 Evaluation Results. The best ASHE 2.0 instance, A4, tied statistically with Slumbot 2017 in heads-up matches and outperformed Slumbot significantly when playing against weaker opponents.

Table 5-4 presents the test results. The first four rows show the performance of A1, A2, A3, and A4 against the 11 opponents in Table 5-3. The last row shows Slumbot's performance against the same opponents for comparison. The last column of the second part of the table contains the agents' performance against Slumbot. Results are marked in blue if an agent played against the opponent in evolution. Performance is measured in milliBig-Blind per hand (mBB/hand). Every pair of agents in the table played at least 20,000 hands against each other. Another 20,000 hands were played if the margin was greater than $20 \%$ of the absolute value of the mean. Thus, approximately 1.5 million hands were played during evaluation. These hands were organized into 1000-hand matches, and ASHE's estimators were reset between consecutive matches. 
Overall, the best of the four ASHE 2.0 instances is A4, followed by A2, A1, and A3 in decreasing order of their average performance. A1 through A3 were mainly used as control group agents.

\section{Performance vs. High-exploitability Opponents}

The results show that ASHE 2.0 is remarkably effective in exploiting weak opponents. Opponent modeling and adaptation allow the agents to quickly adjust their strategies and take advantage of the opponent's weaknesses. In the experiments, eight opponents were considered highly exploitable: HM, SL, CM, CS, LP, TP and RG. These agents are theoretically highly exploitable because their strategies are flawed. However, to exploit their weaknesses effectively, an agent must adopt different counter-strategies that may require entirely opposite actions facing similar game states. Thus, these highly exploitable agents form an effective test group for opponent modeling and adaptation.

As is shown in Table 5-4, the best instance, A4, outperformed SB $40 \%$ to $2300 \%$ when playing against the highly exploitable opponents. The performance gap depends on the type of the weaknesses and the level of exploitability. Generally, the more exploitable the opponent strategy is, the bigger the margin tends to be. Furthermore, the second-best instance, A2, evolved by playing against LA, LP, TP, and TA, was much more effective than SB when facing HM, CM, SL and CS. These results show that ASHE's performance advantage over Nash-equilibrium-based agents is not limited to opponents seen during training. Therefore, the proposed method can build poker agents that are significantly more effective in exploiting weak opponents than top-ranking agents following equilibriumbased strategies. 


\section{Performance vs. Low-exploitability Opponents}

TA, HP, and SB have significantly lower exploitability than the other opponents. All of these opponents were equipped with sound strategies and able to apply sophisticated tactics such as check-raising, slow-playing, continuation bet, etc. The strategy of TA simulates an average amateurish player with a tight-aggressive playing style. HP is designed based on TA, with a number of advanced tactics commonly used by professional players, e.g. floating, pot-control, multi-barrel bluffs, etc., added to its strategy. SB (available at slumbot.com) follows an approximated equilibrium strategy. The version used in the ASHE 2.0 experiments was released on 03/19/2017 with its river strategy improved.

As is shown in Table 5-4, both A4 and A2 defeated TA and HP by a significant margin and tied statistically with SB. Note that HP and SB were not used during training, and both opponents had considerably lower exploitability than the training opponents for A4 and A2.

Thus, these results show that ASHE 2.0, evolved by playing against highly exploitable opponents, can adapt to moderate-to-low exploitability opponents that are not seen during training. The agents can defeat opponents with relatively sophisticated strategies used by amateurish and professional human players and achieve competitive performance against top-ranking Nash-equilibrium-based computer agents.

\begin{tabular}{lll}
\hline & ASHE 1.0 & ASHE 2.0 \\
\hline Tight Aggressive (TA) & $-62 \pm 51$ & $509 \pm 88$ \\
\hline Half-a-Pro (HP) & $-134 \pm 75$ & $278 \pm 59$ \\
\hline Slumbot 2017 (SB) & $-269 \pm 80$ & $5 \pm 61$ \\
\hline
\end{tabular}

Table 5-5: ASHE 2.0 and ASHE 1.0 vs. Low-Exploitability Opponents. Performances are measured by $\mathrm{mBB} /$ hand. ASHE 2.0 is much more effective against strong opponents than ASHE 1.0 due to its ability to extract more subtle patterns via the PRTs and more accurate decision-making based on explicit opponent modeling. 
An important limitation of ASHE 1.0 is its performance against low-exploitability opponents that are not seen during training. How much better can ASHE 2.0 do compared to ASHE 1.0? To answer this question, 20000 hands were played between ASHE 1.0 and each of the three low-exploitability opponents. The games were organized into 500-hand matches, and the LSTM modules in ASHE 1.0 were reset after each match. Table 5-5 presents the results. ASHE 1.0 lost to all three opponents while ASHE 2.0 defeated TA and HP with a large margin and tied with SB. Thus, ASHE 2.0 is much more effective against low-exploitability opponents compared to the previous version.

\section{Performance vs. Dynamic Strategies}

A strategy is dynamic if the action distributions are not fixed given the same game states. For example, most human players follow dynamic strategies. An amateur who lost too much in a cash game may start chasing his loss and become impatient. Consequently, he plays much looser (i.e. entering the pot with more hands) and deviates from his usual strategy noticeably. In fact, ASHE itself follows a dynamic strategy. As more hands are played, ASHE modifies its strategy to exploit the opponent more effectively.

In contrast, rule-based and Nash-equilibrium-based players such as HP and SB follow static strategies. The action distribution for each game state is predefined by rules or equilibria and does not change over time. Since all opponents used in training follow static strategies, how does ASHE perform against opponents with dynamic strategies?

To answer this question, matches were played between ASHE 2.0, ASHE 1.0, and RG, all of which follow dynamic strategies. Each pair of agents played 20000 hands with duplication. The hands were organized into 500-hand matches, and the LSTM modules (and PRTs in ASHE 2.0) in the adaptive agents were reset for each match. 


\begin{tabular}{l|llll}
\multicolumn{1}{r}{ ASHE 2.0 } & ASHE 1.0 & RG & SB \\
\hline ASHE 2.0 & & 581 & 8996 & 5.0 \\
\hline ASHE 1.0 & -581 & & 7984 & 269 \\
\hline RG & -8996 & -7984 & & -2102 \\
\hline SB & -5.0 & 269 & 2102 &
\end{tabular}

Table 5-6: Performance of ASHE against Dynamic Strategies. Green, yellow, and red indicate the agent in the row winning, tying, and losing against the agent in the column with a 0.95 confidence, respectively. ASHE 2.0 exploits dynamic strategies more effectively than SB does. Both ASHE 2.0 and ASHE 1.0 defeats highly exploitable dynamic strategies, e.g. RG, with a big margin.

Table 5-6 presents the results. Performance of SB against each agent is provided for comparison. Both ASHE 2.0 and ASHE 1.0 were more effective in exploiting RG than SB. In addition, ASHE 2.0 defeated ASHE 1.0 with a much bigger margin than SB did. These results show that ASHE 2.0 can adapt to dynamic strategies and exploit them more effectively than equilibrium-based agents. The performance gap tends to get bigger with more exploitable opponents.

To understand how ASHE 2.0 adapts to dynamic strategies, snapshots of ASHE's strategy were taken after every 50 hands in a 500-hand match against RG. Each snapshot strategy was evaluated against RG and the strategy RG chose in the previous 50 hands before the snapshot was taken.

In the sample shown by Table 5-7, RG chose the SL strategy during the first 50 hands. The snapshot strategy at hand 50 achieved maximum performance against SL. However, when facing RG, it did not perform better than SB did. As more hands were played, ASHE's performance against RG improved rapidly. By the end of hand 200, ASHE had played against each strategy RG might choose for at least 50 hands. The performance of the snapshot strategy at hand 250 is four times better than that of SB against RG. The 
performance of snapshot strategies against RG fluctuated around $9000 \mathrm{mBB} / \mathrm{hand}$ after roughly 300 hands.

\begin{tabular}{llllll}
\hline Hand NO. & 50 & 100 & 150 & 200 & 250 \\
\hline Recent RG Strategy & SL & HM & CM & CS & HM \\
\hline Snapshot vs. Recent & 1000 & 22211 & 21723 & 1868 & 18921 \\
\hline Snapshot vs. RG & 1937 & 4890 & 5708 & 6223 & 8513 \\
\hline & & & & & \\
\hline Hand NO. & 300 & 350 & 400 & 450 & 500 \\
\hline Recent RG Strategy & SL & CS & CM & CS & HM \\
\hline Snapshot vs. Recent & 999 & 3109 & 16987 & 3712 & 17617 \\
\hline Snapshot vs. RG & 8882 & 9195 & 9121 & 9241 & 8916 \\
\hline
\end{tabular}

Table 5-7: Snapshot Strategy Analysis. ASHE 2.0 gradually discovered a strategy with balanced performance against the four highly exploitable strategies of the opponent RG. After 300 around hands, performance stabilized at approximately $9000 \mathrm{mBB} / \mathrm{hand}$.

Note that when playing against HM, the snapshot at hand 250 and hand 500 were noticeably less effective than the snapshot at hand 100, although all three snapshots were taken right after 50 hands against the HM strategy (red). Similarly, the snapshot strategy at the 400th hand was less effective against CM than the snapshot at hand 150 (blue). On the other hand, as the match proceeded, snapshot strategies became increasingly effective against CS (green). The performance against RG also improved with more hands.

These results indicate that when playing against RG, ASHE did not switch its strategy as RG did after every 50 hands, neither did it attempt to identify the strategy RG used recently and switch to the most effective counter-strategy previously found. Instead, ASHE were able to find a strategy that exploited RG based on the overall distribution of its moves across all the strategies it might randomly choose. That strategy, while being close to static after a few hundred hands, was much more effective in exploiting RG compared to SB's approximated equilibrium strategy. 
Adaptation in matches between ASHE 2.0 and ASHE 1.0 appeared to be similar. At the beginning, the two adaptive agents changed their behaviors drastically, racing for a strategy to better exploit the opponent. As more hands were played, the strategies of both agents started converging. After a few hundred hands, the performance became stable.

Thus, ASHE 2.0 adapts to dynamic strategies by reacting to their overall action distribution rather than just following the recent moves.

\section{Training Opponent(s) and Performance}

Results in Table 5-4 also provide insights into how opponents should be selected and arranged for training/evolution. First, the performance of A3 was much worse than the other three instances when facing opponents whose strategies were fundamentally different from those seen during evolution. For instance, CM and CS are fundamentally different from any opponent used in A3's training. Consequently, A3's performance against these agents were much lower than that of A1, A2, or A4. Moreover, A3's strategy did not perform well against stronger opponents that were not seen during training. In contrast, instances evolved by playing against a reasonably diversified set of strategies, e.g. A1, A2, and A4, could adapt to HP or SL, whose strategies were superior to any strategies seen during evolution.

These results indicate that a reasonable level of diversity of opponent strategies during evolution is necessary for evolving strong adaptive agents. It is unlikely for an agent that has never seen maniac-style bluffing to exploit it effectively. In other words, the agents should be exposed to different types of weaknesses through evolution. With such diversity, however, agents can generalize remarkably well to opponents that are not seen in training.

While exploitable patterns can be categorized into many types, agents with these patterns may have different level of exploitability. For example, TP and SL both tend to 
underestimate the value of their hands and fold more often than they should. However, SL is extremely cautious and only plays the absolute best hands, and TP continues with a noticeably wider range. Suppose multiple agents with the same type of weaknesses are available for training, which works better as a training opponent?

In the experiments, A2 and A1 were evolved by playing against opponents with the same types of weakness. A2's training opponents, i.e. LA, LP, TP, and TA, were less exploitable compared to A1's training opponents, i.e. HM, SL, CM, and CS. As a result, A2 achieved consistently better performance than A1 when playing against opponents that had not been seen during evolution. Therefore, the level of exploitability of opponent strategies during evolution is another factor that influences performance. Moderately exploitable opponents tend to be more effective as training opponents than a highly exploitable opponents with similar types of weakness.

Experimental results also indicate that combining training opponents with high and low level of exploitability can improve performance. In particular, highly exploitable training opponents can be used in early generations. Once the agents start demonstrating adaptive behaviors, less exploitable training opponents can be introduced. For instance, both A2 and A4 played against LA, LP, TP, and TA during training. Nevertheless, A4 were evolved by first playing against a group of more exploitable opponents with similar types of weakness, i.e. HM, SL, CM and CS. In matches against opponents that were not seen by both agents during training, A4 performed consistently better than A2.

In addition, using highly exploitable agents at the early stage of evolution can improve training efficiency, because they are more sensitive to exploitation and provide more incentive for the agents to explore adaptive behaviors. In comparison, exploitable patterns of stronger opponents tend to be more subtle and offer less reward even if fully 
exploited. These subtle patterns are great training examples after enough generations, but may be overwhelming for the agent population in the beginning.

The above observations and findings show that the proposed approach is effective in building high-quality adaptive agents for HUNL. They also provide useful principles for its application and improvement in the future.

\subsection{DisCUSSION AND CONCLUSION}

ASHE 2.0 introduces two major improvements to the previous version: (1) Pattern Recognition Trees/PRTs and (2) explicit opponent modeling. The PRTs are tree structures whose nodes correspond to the game states. They collect statistical data on the opponent's strategy and serve as a feature extractor of the opponent model. Input features derived from the node stats in PRT are sent to the LSTM modules, which evaluate fold equities and showdown values. The PRTs improve performance in two ways. First, they extract useful features on the opponent's strategy from the game history. Second, they reduce the complexity of the task for the LSTM modules, thus allowing the networks to be smaller and evolution more efficient.

Explicit opponent modeling refers to predicting opponent actions, estimating hand strength, etc. These predictions can be used to estimate the utility of each possible action, providing a more accurate method for deciding the next moves. ASHE 2.0 evaluates fold equity and showdown value with LSTM modules, which take inputs derived from raw game states and the PRT node stats.

Experimental results demonstrates that ASHE 2.0 is significantly more effective than cutting-edge equilibrium-based agents in exploiting weak opponents. In addition, ASHE 2.0 can adapt to opponents that are not seen during training, even if they are much

stronger than the training opponents. In particular, ASHE 2.0 tied statistically in heads-up 85 
matches with Slumbot 2017, an upgrade of the equilibrium-based agent used in the ASHE 1.0 experiments. The PRT-LSTM-based opponent model is effective in matches against both static and dynamic strategies. Experimental results show that ASHE 2.0 outperforms Slumbot by significant margin facing dynamic opponents with different level of exploitability.

Empirical results also provide insights into how opponents should be selected and arranged during evolution. Training opponent should be reasonably diversified to ensure generalizability. Moderately exploitable opponents in training tend to yield better results than highly exploitable opponents with similar types of weakness. Nevertheless, highly exploitable opponents can be used in early generations to encourage adaptive behaviors.

While ASHE 2.0 achieved much higher performance than the previous version against strong opponents that are not seen during training, it can be further improved in several ways. Chapter 6 to 8 introduce various extensions of ASHE 2.0 and investigate their influence on performance. Chapter 6 focuses on the PRTs, Chapter 7 focuses on action utility estimation, and Chapter 8 introduces the Hand Range Estimators. The overall result will be a version of ASHE that will defeat a wide range of opponents, including Slumbot 2017, in heads-up matches. 


\section{Chapter 6: Advanced PRT}

In ASHE 2.0, the Pattern Recognition Tree serves as a feature extractor for the opponent model and plays an important role in capturing exploitable patterns in the opponent's strategy. However, the PRTs in ASHE 2.0 have two limitations: (1) they cannot provide useful node stats when facing a new opponent or an infrequent game state, and (2) they do not take community cards into consideration.

This chapter introduces advanced PRTs, which addresses the above limitations. Advance PRT entails two techniques: the default PRT (Section 6.1), which models the actions of an unknown opponent, and the board-texture-based PRT (Section 6.2), which is able to capture exploitable patterns based on the community cards. Section 6.3 presents and analyzes experimental results on ASHE 2.1, the upgraded version with advanced PRT.

\subsection{Default PRT}

The default PRT allows the agents to make better decisions in the early stage of a match when node stats are unreliable, thus improving overall performance. This technique is also a prerequisite for the PRTs to model the opponents based on board textures, because collecting reliable node stats on such specific game states takes more hands, and decision quality must be guaranteed before node stats become reliable.

\subsubsection{Motivation}

The PRTs in ASHE 2.0 are updated in a lazy fashion. They are initialized with a root node that represents one of the two initial states of the game. Nodes representing the other game states are inserted to the PRTs when the game states occur in the match. Even if the node corresponding to a game state already exists in the PRTs, node stats cannot be used to derive inputs for the LSTM estimators before the game state occurs enough times 
to generate meaningful data. Thus, in the early stage of a match, the agent have to make many decisions essentially without the support of the PRTs.

In ASHE 2.0, if node stats are not reliable, a set of default values are sent to the estimators in place of the features derived from the node stats. Different default values are selected for each betting round. These values are estimated based on domain knowledge and empirical results. They do not provide information on the opponent's strategy, neither do they correspond to any specific game state. Consequently, ASHE's strategy in the early stage of a match, i.e. the first 50 to 100 hands, tends to be unstable and flawed, reducing overall performance.

Moreover, the more nodes the PRTs have, the longer it takes to collect reliable node stats. Hence, the low quality of default values makes it infeasible to expand the PRTs to represent more specific game states. In fact, it is the main reason for the PRT nodes in ASHE 2.0 to ignore the community cards.

The key to address the above problem is to provide high quality default inputs for the LSTM estimators when node stats are unreliable. While it is impossible to extract exploitable patterns in a specific strategy without sufficient observation, a player can still make good decisions in the early stage of a match.

When facing a new opponent at the table, a human player usually adopts an initial strategy based on his/her experience from games against other players. As more hands are played, the player learns about the opponent and adapts his/her strategy accordingly. Similarly, default inputs for the LSTM estimators can be generated from games played against other opponents. Such inputs may not represent the strategy of the current opponent. However, they allow the agent to make decisions at infrequent states that work well enough against most opponents. 
Default PRT is designed to provide such inputs. It is used in combination with the opponent-specific PRTs introduced in Chapter 5, allowing the agents to make good earlymatch decisions. The next subsection details this technique.

\subsubsection{Construction and Application}

A default PRT has the same structure as the regular PRT and maintains the same set of node stats listed in Subsection 5.1.2. However, they represent different strategies.

The regular PRTs are built for each opponent from scratch. As more hands are played, the regular PRTs grow, and the node stats become increasingly reliable. They reflect the strategy of the current opponent. In contrast, the default PRTs are built during training. They do not change in a match, and their node stats are used to derive input features for the LSTM estimators when the regular PRTs cannot provide reliable node stats. The default PRTs represent the general strategy ASHE adopts when facing a new opponent or game state.

\section{Construction}

Like the regular PRTs, the default PRTs are constructed through playing against other agents. When evolving agents with default PRTs, each generation is divided into two sessions: a preliminary session and an evaluation session.

In the preliminary session, the agents play against the default opponent(s). The entire session is treated as one match, and the PRTs are not reset even if the agents play against multiple opponents. Default values are used when facing new game states like the ASHE 2.0 baseline. At the end of the session, the PRTs are saved as the default PRTs.In the evaluation session, the agents play against their opponents using both the default PRTs 
and the regular PRTs. The performance of the agents is measured by their Average Normalized Earnings/ANEs in the evaluation session only.

Since the node stats in the default PRTs are accumulated in games played against the default opponent(s), choosing the right default opponent(s) is important for achieving high performance. Ideally, the default opponent(s) should encourage counter-strategies that perform well against most players. Thus, highly exploitable agents with unique weaknesses tend to be less effective than agents with low exploitability. However, it is possible to form an effective default opponent set by selecting a group of highly exploitable opponents with common and diversified strategies.

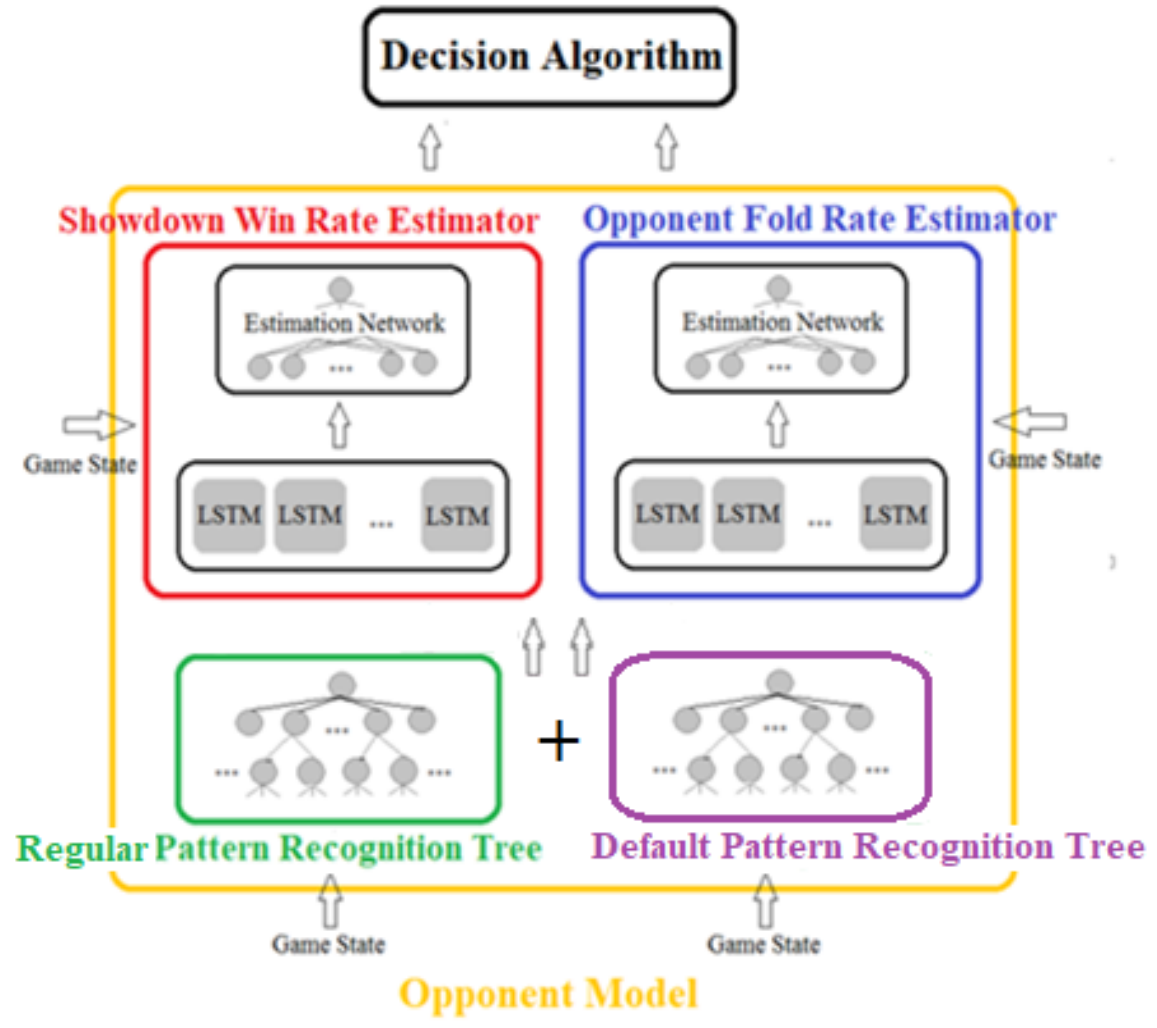

Figure 6-1: ASHE with Default PRTS (ASHE 2.1). Node stats from the default PRTs and the regular PRTs are combined linearly, allowing the LSTM estimators to make more accurate prediction facing new opponents or infrequent game states. 


\section{Application}

Figure 6-1 presents the architecture of ASHE 2.1, an upgrade of the baseline equipped with the default PRTs. The game states are sent to the default PRTs for tracking actions in the game and retrieving node stats. Unlike the regular ones, the default PRTs are not updated at the end of each hand. Given a game state, the node stats are retrieved from each of the two PRTs. The stats are linearly combined based on the state frequency $f$ (as defined in Subsection 5.1.2) and the normalization factor $\lambda$ :

$$
v= \begin{cases}\frac{f}{\lambda} v_{\mathrm{R}}+\left(1-\frac{f}{\lambda}\right) v_{\mathrm{D}} & (f<\lambda) \\ v_{\mathrm{R}} & (f \geq \lambda)\end{cases}
$$

where $v$ is the node stats value used to derive input features for the LSTM estimators, $v_{\mathrm{R}}$ and $v_{\mathrm{D}}$ are the node stats from the regular PRT and the default PRT, respectively. The normalization factor $\lambda$ can be adjusted based on the depth of the nodes. The deeper the nodes are, the more difficult it is for the regular PRTs to collect reliable data. Hence, $\lambda$ can be smaller for deeper nodes.

If the node stats in the default PRT is also unreliable, the default features are sent to the LSTM estimators. However, such cases are extremely rare and do not influence overall performance.

Thus, the default PRTs provide node stats representing the default opponent(s) to derive input features for the LSTM estimators. They allow the estimators to make reliable predictions even if ASHE has little information on the opponent.

\subsection{BOARD-TEXTURE-BASED PRT}

With the default PRTs in place, the LSTM estimators no longer rely solely on the regular PRT node stats for inputs features. Therefore, the regular PRTs are allowed more 
time to collect reliable node stats. This means the PRT nodes can represent more specific game states and capture more subtle patterns in the opponent strategies.

This section introduces board-texture-based PRT, which maintains node stats for game states defined by the action sequence and the patterns in the community cards. The board-texture-based PRT and the default PRT technique are applied together to build the advanced PRTs in ASHE 2.1.

\subsubsection{Motivation}

The raw game state of HUNL is defined by the action sequence, the private cards, and the community cards. The nodes of the PRTs in ASHE 2.0 only represent the action sequences. What should be added to make the PRT more effective for opponent modeling and exploitation?

The private cards certainly play an important role in the game. Nevertheless, they are not a viable option to be represented by the PRT nodes. The opponent strategy is not related to ASHE's private cards. The opponent makes decisions based on its own private cards, the action sequence, and the community cards. Knowing the opponent's private cards is very helpful in modeling the opponent strategy. However, a large portion of hands end without a showdown, and the opponent's private cards are never revealed. Hence, it is difficult to extract or update the node stats.

The community cards, a.k.a. the board, is critical for making decisions in HUNL. Many exploitable patterns may be closely related to the texture of the board. For instance, some players tend to overvalue flush and/or straight draws and semi-bluff excessively. Taking the board into consideration is necessary to discover and exploit such weaknesses. The biggest challenge to introduce the community cards as part of the game states 
represented by the PRT nodes is the huge number of possible boards. There are over two million possible boards, and it is impossible to create nodes for all of the combinations.

ASHE 2.1 addresses this problem by clustering the boards at each betting round based on their texture (e.g. if the board is paired, connected, suited, etc.). Game states are defined using clusters instead of specific cards. This approach simulates what human players do. Suppose two cards of the same suit show up on the flop, a human player may notice that the board allows flush draws, but the specific ranks of the suited cards are often ignored, unless they are part of another feature that is related to the ranks. Since there are no more than a dozen of interesting board texture, they can be included in the game state to represent the community cards. Thus, board-texture-based PRTs are able to capture patterns that are directly related to the board while collecting reliable node stats quickly.

\subsubsection{Implementation}

Both the opponent-specific PRTs and default PRTs in ASHE 2.1 are board-texturebased. This subsection introduces the structure of the board-texture-based PRTs and specifies the method to generate the board texture clusters.

\section{Board-texture-based PRT Structure}

Figure 6-2 illustrates the structure of a board-texture-based PRT. Compared to the PRTs in ASHE 2.0, the board-texture-based PRTs have one more type of nodes: the board nodes. Each of them represents a cluster of boards. Since the board nodes are not decision points, they do not have node stats. The board-texture-based PRTs work in the same way as the ASHE 2.0 PRTs do, with one exception: when the community cards are dealt, the board is associated with one of the board texture clusters, and the board node representing that cluster is visited. If the board node does not exist, one will be added to the tree. 


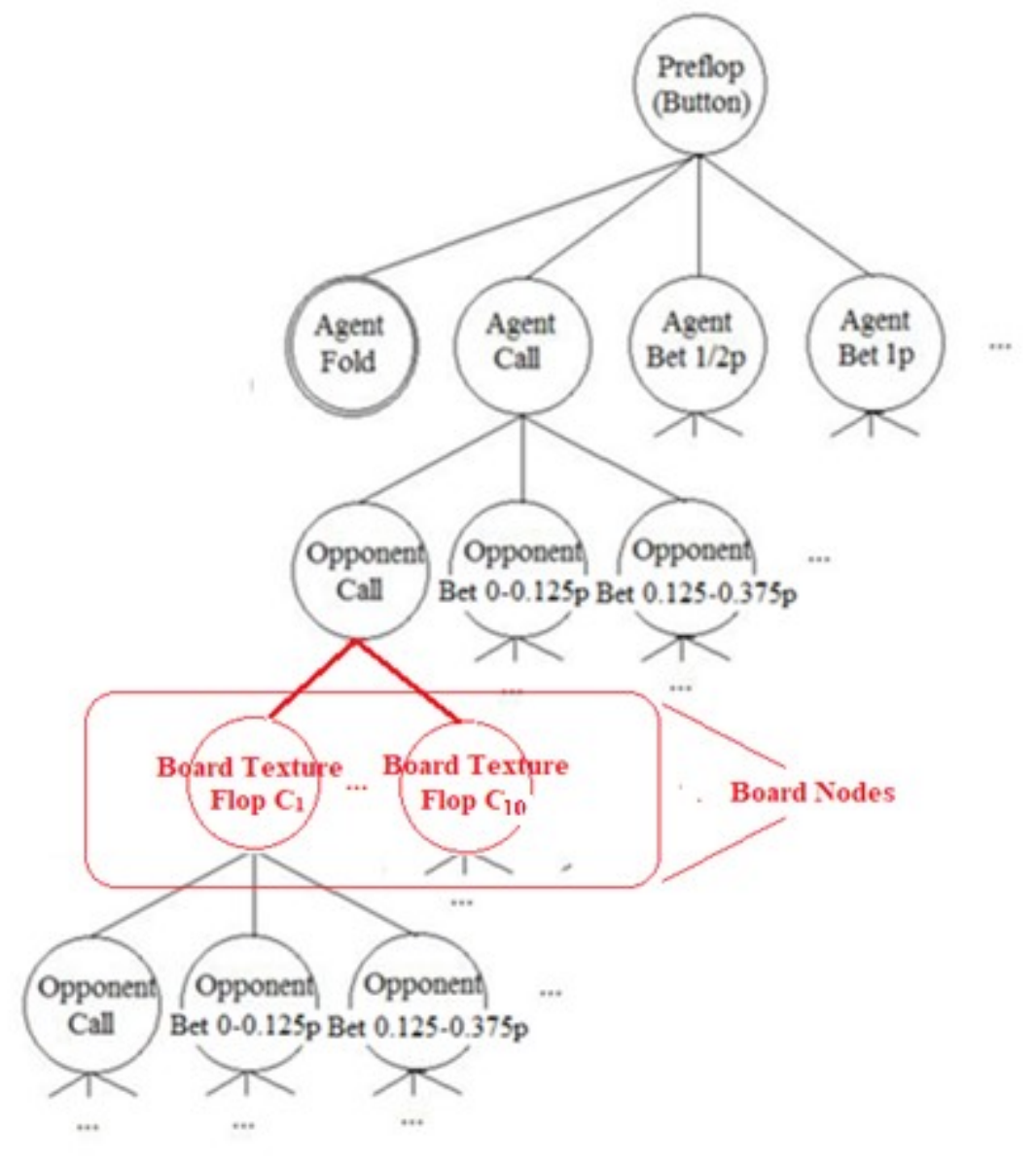

Figure 6-2: A Board-Texture-Base PRT. Board nodes are added to represent the texture of the board, allowing the PRT to capture subtle patterns that are directly related to the community cards.

\section{Board Texture Clusters}

Human players usually categorize the boards based on individual features, e.g. if the boards contain pair(s), suited cards, connected cards, etc. Thus, the boards can be represented by tuples, with each attribute corresponding to one of these features. Table 61 provides the definition of the most important board textures and how to represent them quantitatively. The features marked with "*” should take community card(s) in the future betting round(s) into consideration to represent the draws. 


\begin{tabular}{ll}
\hline Feature & Definition/Representation \\
\hline Pairedness & The number of pairs $(0,0.5$, or 1, indicating 0,1 or 2 pair(s) $)$ \\
\hline Suitedness* & The probability for a random hand making a flush with the board \\
\hline Connectivity* & The probability for a random hand making a straight with the board \\
\hline
\end{tabular}

Table 6-1: Board Textures. Pairedness, suitedness, and connectivity are three important features that define the texture of the board. Pairedness is normalized for clustering.

Since there are numerous combinations of values from these features, board tuples cannot be used to define the board nodes in the PRTs directly. Instead, the tuples at each betting round are clustered using K-means algorithm.

There are a total of 22,100 possible boards on the flop, 270,725 on the turn, and 2598960 on the river. The cluster numbers for each betting round should hit a balance between the average time for collecting reliable PRT node stats and the cluster quality (i.e. sum of square error). Cluster numbers in ASHE 2.1 are:

$$
K_{r}=\left\lceil\lg \left(N_{r}\right)\right\rceil
$$

where $\lg$ is logarithm function (with base 10 ), $K_{\mathrm{r}}$ and $N_{\mathrm{r}}$ indicate the number of clusters and possible boards for betting round $\mathrm{r}$, respectively. Thus, there are five clusters for the flop, six for the turn, and seven for the river. Clustering results are precomputed and hashed for fast retrieval. Generally, the boards are clustered based on how wet they are (the possibility of randomly selected private cards making a big hand such as a straight, a flush, etc.), and the types of big hands that are possible. Empirical results show that clusters generated in this method are effective in covering and distinguishing most board textures.

The board-texture-based PRTs introduced in this section allow ASHE to capture exploitable patterns related to the community cards, thus improving performance against low exploitability opponents significantly. 


\subsection{EXPERIMENTAL RESULTS}

This section presents and analyzes experimental results on ASHE 2.1, which is an upgrade version of ASHE 2.0 with default PRTs added to the opponent model. Both the default PRTs and the opponent-specific PRTs in ASHE 2.1 are board-texture-based. For convenience of discussion, such PRT settings will be referred to as advanced PRT in the rest of this dissertation.

\subsubsection{Experimental Setup}

Although the PRTs are not optimized through evolution, neural network modules must be re-trained to utilize additional information from the advanced PRTs. Parameters are set to the same values as in Table 5-2.

The normalization factor $\lambda$ (as defined in Subsection 6.1.2) should decrease for nodes representing longer action sequences to keep a balance between node stats from the default PRTs and the regular PRTs. In the experiments, $\lambda$ was set to $50,20,10$, and 5 for preflop, flop, turn and river nodes, respectively.

The preliminary session for each generation contained 5000 hands. These hands were chosen from the board texture clusters with equal probability to ensure most nodes in the default PRTs contain reliable node stats.

The same agents specified in Table 5-3 were used for evolution and evaluation. The match format were also the same, i.e. 1000 duplicated hands per match, $\$ 50 / \$ 100$ stake, and \$20000 stacks refilled every hand.

The opponent-specific PRTs in the ASHE 2.1 agents were reset to their initial states at the start of each match and before switching position to replay the duplicated hands in the match. The default PRTs remain unchanged after the preliminary session. 


\subsubsection{Results and Analysis}

Two instances of ASHE 2.1 were evolved using different default opponents in the preliminary session. The default opponents for A1 are LP, LA, TP, and TA, a group of opponents with diversified exploitable patterns. The default opponent for A2 was A2 itself, i.e. the default PRTs were built by playing with the champion from the previous generation.

Table 6-2 presents the results. All performances are measured in $\mathrm{mBB} /$ hand. The performance of Slumbot 2017 and ASHE 2.0 (best instance) are listed for comparison. During evaluation, both ASHE 2.1 instances played at least 20,000 duplicated hands against each opponent. If the standard deviation is bigger than $20 \%$ of the absolute value of the mean, another 20,000 hands were played.

Both of the ASHE 2.1 instances were evolved in the same way as the ASHE 2.0 instance. In the first 200 generations, the agents were evolved against SL, HM, CM, and CS. In the next 300 generations, LP, LA, TP and TA were used instead. Performances against agents used during training are marked in blue.

\begin{tabular}{|c|c|c|c|c|c|c|c|}
\hline & & $\mathrm{HM}$ & $\mathrm{CM}$ & SL & CS & RG & HP \\
\hline ASHE 2.1 & (A1) & $40401 \pm 5157$ & $44932 \pm 5116$ & $999 \pm 1.5$ & $10140 \pm 1566$ & $8622 \pm 699$ & $364 \pm 57$ \\
\hline ASHE 2.1 & (A2) & $39998 \pm 5361$ & $44295 \pm 4994$ & $999 \pm 1.2$ & $10523 \pm 1594$ & $8598 \pm 703$ & $381 \pm 60$ \\
\hline ASHE 2.0 & & $42333 \pm 6037$ & $46114 \pm 4985$ & $999 \pm 1.4$ & $9116 \pm 1403$ & $8996 \pm 721$ & $278 \pm 59$ \\
\hline \multirow[t]{2}{*}{ SB } & & $4988 \pm 881$ & $2761 \pm 355$ & $702 \pm 59$ & $4512 \pm 472$ & $2102 \pm 393$ & $152 \pm 50$ \\
\hline & & LA & LP & $\mathrm{TP}$ & TA & SB & ASHE 2.0 \\
\hline ASHE 2.1 & (A1) & $24332 \pm 1298$ & $17888 \pm 685$ & $1601 \pm 102$ & $589 \pm 79$ & $57 \pm 49$ & $41 \pm 46$ \\
\hline ASHE 2.1 & (A2) & $19021 \pm 976$ & $15524 \pm 302$ & $1525 \pm 98$ & $535 \pm 76$ & $69 \pm 50$ & $62 \pm 43$ \\
\hline ASHE 2.0 & & $20005 \pm 1006$ & $15372 \pm 634$ & $1488 \pm 91$ & $509 \pm 88$ & $5 \pm 61$ & - \\
\hline SB & & $2449 \pm 460$ & $623 \pm 41$ & $603 \pm 51$ & $284 \pm 49$ & - & $-5 \pm 61$ \\
\hline
\end{tabular}

Table 6-2: ASHE 2.1 Evaluation Results. Both of the ASHE 2.1 instances defeated Slumbot 2017 and ASHE 2.0, and outperformed ASHE 2.0 in matches against opponents with relatively low exploitability. 
The rest of this section summarizes key discoveries and conclusions from these results. First, both A1 and A2 outperformed ASHE 2.0 when playing against opponents of relatively low exploitability. In particular, both instances defeated SB with statistically significant margin. These results indicate that the advanced PRTs in ASHE 2.1 are particularly effective against strong opponents for their ability to extract complex patterns in opponent strategies.

Second, both ASHE 2.1 instances exploited RG effectively and defeated ASHE 2.0 in heads-up matches, demonstrating remarkable generalizability to dynamic strategies that are not seen during training.

Third, both ASHE 2.1 instances are effective against opponents with different level of exploitability. Thus, the default opponent(s) can be either dynamic (A2) or static (A1). A group of agents with diversified and highly exploitable patterns and adaptive agents with relatively low exploitability can both serve the purpose, as long as they allow ASHE to explore different paths in the default PRTs and develop a good default strategy.

Fourth, agents with advanced PRTs tend to be more effective against opponents that are similar to their default opponent(s). A1 achieved the highest performance against LA, LP, TP, and TA, all of which were used as default opponents for the instance. A2 performed best against ASHE 2.0, SB, and HP, whose low-exploitability strategies are similar to A2 itself. In contrast, when playing against $\mathrm{HM}$ or $\mathrm{CM}$, it took longer for the ASHE 2.1 instances to adapt to these highly exploitable opponents than the ASHE 2.0 instance, resulting in slightly worse performance.

In sum, advanced PRTs allow ASHE to capture board-based patterns in opponent strategies. The ASHE 2.1 is particularly effective against low-exploitability opponents defeating both Slumbot 2017 and ASHE 2.0 in heads-up matches. 


\section{Chapter 7: Recurrent Utility Estimation}

In ASHE 2.0, the decision algorithm evaluates the expected utilities for available actions and selects the action with the highest utility. Hence, utility estimation is critical to achieving high performance. As is introduced in Subsection 5.1.3, utility estimation in ASHE 2.0 is based on the assumption that when ASHE bets or raises, the opponent either folds or calls; if the opponent calls, the game goes to a showdown without more chips committed to the pot. Clearly, the above assumption may not always hold, especially in early rounds of betting, i.e. preflop and flop. This assumption simplifies utility estimation, thus allowing faster decision. However, it can make utility estimation inaccurate, leading to suboptimal decisions. Thus, this chapter introduces Recurrent Utility Estimation, which takes possible future moves into consideration and estimates action utilities more accurately. ASHE 2.2 was built using this technique. Experimental results demonstrate that the algorithm improves performance against opponents with different level of exploitability.

\subsection{RECURRENT UTILITY ESTIMATION}

To evaluate the utilities of available actions more accurately, future moves must be taken into consideration. For example, slow playing (checking/calling instead of betting or raising to induce opponent aggression) a big hand is profitable only if the opponent is likely to bet/raise in the future. Similarly, floating (i.e. calling with a weak hand in order to bluff later) is a viable option when the opponent is likely to fold under pressure in the next betting round(s).

Recurrent Utility Estimation relies on advanced PRTs and the LSTM estimators to predict future moves from the opponent and evaluate showdown values at the terminal states. Figure 7-1 outlines the algorithm. 


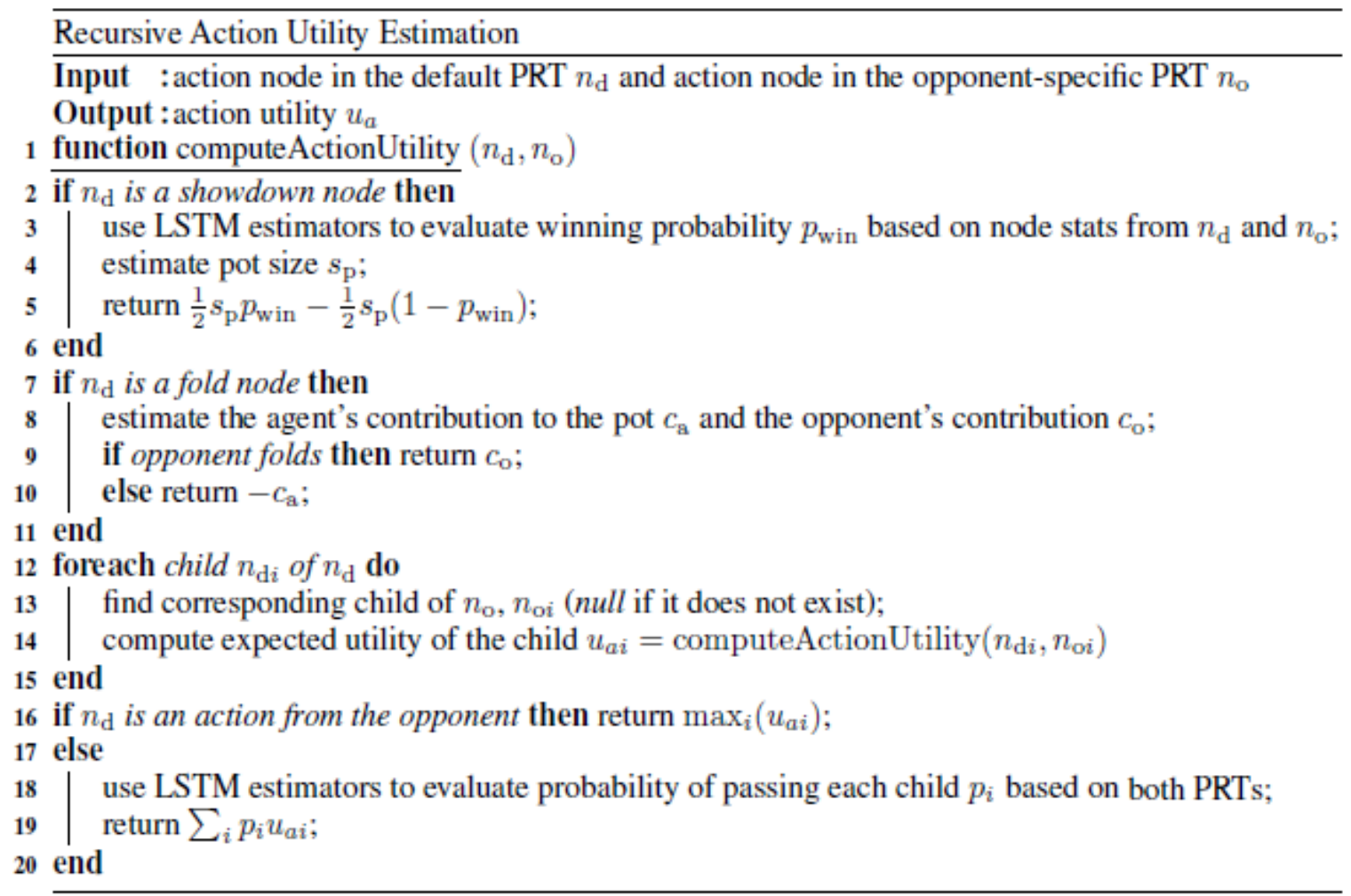

Figure 7-1: Recurrent Utility Estimation. Action utilities are estimated based on current game state and possible future moves, making the estimation much more accurate.

The algorithm estimates action utilities by doing a depth first search on the default and regular/opponent-specific PRTs. When reaching a terminal state, i.e. a leaf node, the utility can be computed directly (line 2-11). The pot size at a terminal state is estimated based on the action sequence to reach that state (line 4). It is impossible to know the opponent's actual bet/raise size in advance. Hence, the mean of the bucket (as defined in Subsection 5.1.2) that corresponds to the presumed action is used instead. In the case of a showdown, the algorithm assumes the output from the Showdown Win Rate Estimator to be the true probability of winning (line 3). If a player folds, utility is computed based on the amount of chips committed by each player (line 9 and 10). 
Given a node that represents a non-terminal state, the algorithm first computes the utility for each child node recursively. In other words, the algorithm estimates the utilities assuming each possible move from the next player to act.

If ASHE is the next player to act, the algorithm assumes that ASHE will select the action with the highest utility and returns that values (line 16). If the opponent is the next player to act, a new LSTM module called the Opponent Action Rate Estimator (i.e. OARE, introduced in Section 7.2), predicts the probability of each opponent action. The utility of this non-terminal state is the sum of all utilities of the child nodes, weighted by their corresponding probability (line 18-19).

As the algorithm visits each non-leaf node, the states of the LSTM modules are saved. Then, inputs derived from the raw game state and the nodes stats in the default and regular PRT are sent to the SWRE and the OARE. In the rare case where the node stats in both PRTs are unreliable, utility is estimated using the ASHE 2.0 decision algorithm. The states of the LSTM modules are restored after the algorithm finishes visiting the node.

When applying Recurrent Utility Estimation in combination with advanced PRTs, the algorithm may run into board nodes. While community cards in future betting rounds are not known, the utility can be approximated using the sum of the utilities given each cluster, weighted by their respective probabilities.

For the first two betting rounds, i.e. preflop and flop, computing the utilities for all possible paths can be very costly. Therefore, a Monte Carlo method is used to generate random boards, and the algorithm returns the average utility of all samples. Note that different boards may fall into the same cluster, and the utility only needs to be computed once for each cluster/board node. As for the turn, since there is only a single community card to come, the algorithm traverses all possible paths. Thus, the Recursive Utility 
Estimation algorithm gives more accurate estimation on action utilities, allowing the agents to make better decisions.

\subsection{ASHE 2.2 ARCHITECTURE}

ASHE 2.2 combines the advanced PRTs with Recursive Utility Estimation. Figure 7-2 illustrates the architecture of ASHE 2.2. The Opponent Fold Rate Estimator in the previous version is replaced with the Opponent Action Rate Estimator, which predicts the probabilities of each available action from the opponent.

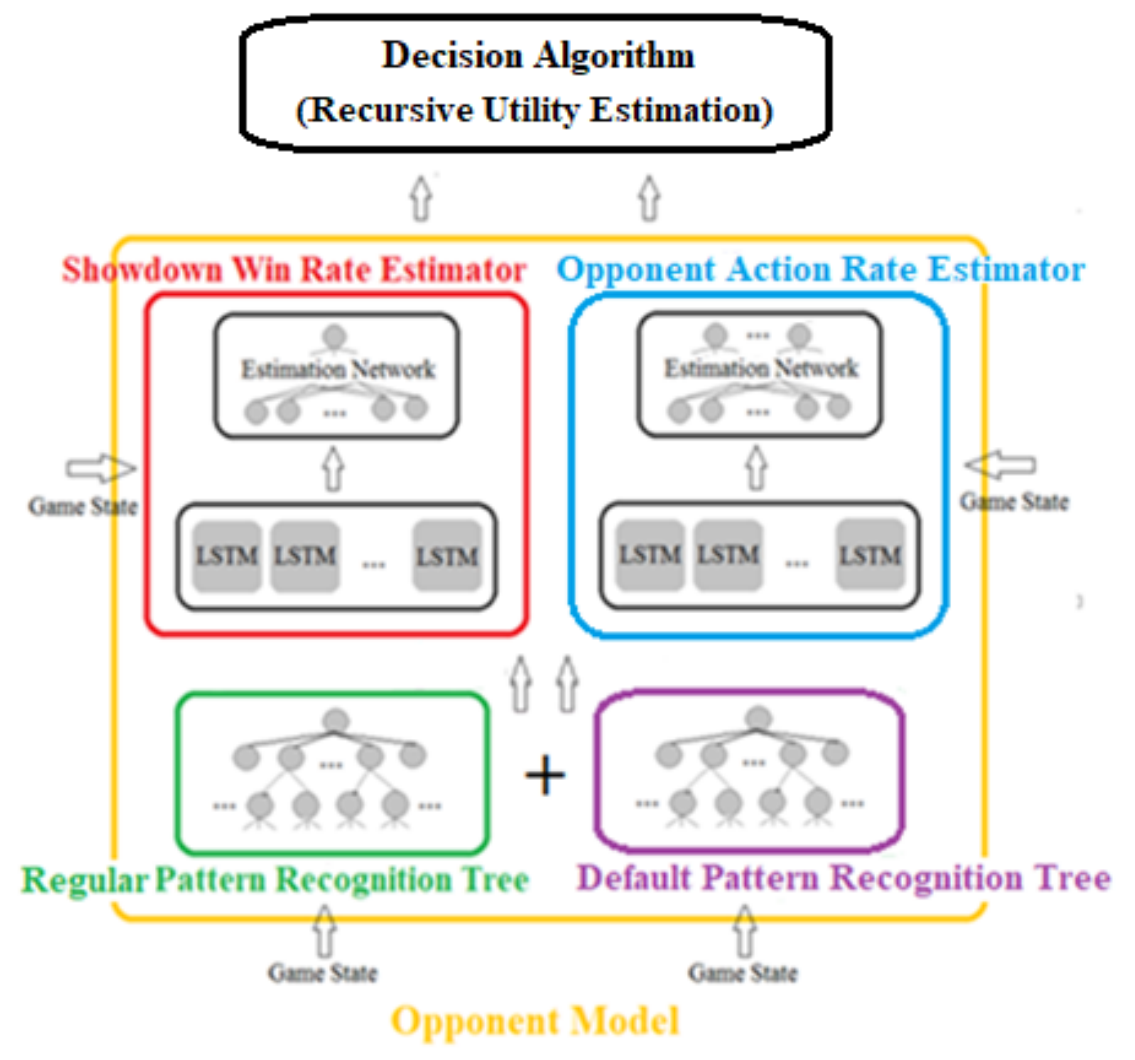

Figure 7-2: ASHE with Recursive Utility Estimation (ASHE 2.2). Opponent Fold Rate Estimator used in the previous version is replaced by the Opponent Action Rate Estimator (OARE) to predict probability of different opponent actions for Recursive Utility Estimation. 
Like the OFRE, The OARE consists of a single layer of LSTM blocks and a fully connected feed-forward estimation network. The output layer of the OARE estimation network contains nine nodes, corresponding to fold, check/call, and bet/raise in each of the seven bet-size buckets. These outputs go through a softmax function before being sent to the decision algorithm. An output is skipped in the softmax if the corresponding action is not available to the opponent.

To support opponent action prediction other than folding, the PRT node stats are expanded as follows:

- $\quad$ State Frequency $(f)$, showdown counts $\left(\mathrm{c}_{\mathrm{sd}}\right)$, and showdown strength $(\bar{s})$ remains the same as defined in Subsection 5.1.2. "Fold counts" in 5.1.2 $\left(c_{\mathrm{of}}\right)$ is now referred to as the "eventual fold count", not to be confused with the new fold counts $c_{\mathrm{f}}$. These node stats are maintained at every non-leaf node as before.

- Fold Counts $\left(c_{\mathrm{f}}\right)$ : the number of times the opponent folding its hand as the next action.

- Check/Call Counts $\left(c_{\mathrm{c}}\right)$ : the number of times the opponent checking or calling as the next action.

- Bet/Raise Counts $\left(1-7, c_{\mathrm{b} 1}-c_{\mathrm{b} 7}\right)$ : the number of times the opponent betting or raising in each of the seven buckets as the next action.

The new node stats, i.e. $c_{\mathrm{f}}, c_{\mathrm{c}}$, and $c_{\mathrm{b} 1}$ to $c_{\mathrm{b} 7}$, are maintained only in nodes that represent a game state where the opponent is the next to act. These nodes correspond to ASHE's actions rather than those of the opponent (i.e. ASHE's decision points).

During runtime, the input features for the SWRE remain the same as specified by Table 5-1, although the feature "fold rate" is renamed as "eventual fold rate". 
The OARE takes all the input features that are sent to the OFRE in the previous versions, with the exception of the eventual fold rate. In addition, it also takes fold rate, call/check rate, and bet/raise rates for each bucket as its inputs. These features are defined as their corresponding count divided by the state frequency $(f)$. In PRT nodes where these counts are not maintained (i.e. ASHE is the next to act), the features are set to zero. All features derived from the node stats in the default PRT and regular PRT are linearly combined as specified in Subsection 6.1.2.

Given a game state, the ASHE 2.2 decision algorithm performs Recursive Utility Estimation for each available action. In the rare case where the node stats in both PRTs are unreliable or the action node does not exist, the utility of that action is estimated by the ASHE 2.0 decision algorithm. The OARE and the SWRE are restored to the current states (Subsection 5.1.2) after all estimations complete.

Thus, the ASHE 2.2 architecture utilizes the node stats in the advanced PRTs to predict opponent actions. It is essentially another step towards explicit opponent modeling, thus allowing decision-making to be more accurate.

\subsection{EXPERIMENTAL RESULTS}

This section presents and discusses experimental results on ASHE 2.2, which integrates advanced PRTs with Recursive Utility Estimation.

\subsubsection{Experimental Setup}

Table 7-1 lists key parameter settings for the ASHE 2.2 experiments. Evolution was extended to 750 generations. Sessions evolving the OARE contained twice as many generations as those for the SWRE. Monte Carlo sampling size was set to 100 for the first two betting rounds, which keeps a good balance between fitness and training efficiency. 


\begin{tabular}{llll}
\hline Parameter & Value & Parameter & Value \\
\hline Number of Generation & 750 & LSTM Block Size & 10 \\
\hline Generation(s) per Session & $50 / 100$ & Blocks per Module & 50 \\
\hline Population Size & 50 & Estimation Net Input Size & 500 \\
\hline Survival Rate & 0.30 & Estimation Net Hidden Layer & 1 \\
\hline Mutation Rate (initial/final) & $0.25 / 0.05$ & Estimation Net Hidden Nodes & 50 \\
\hline Mutation Strength (initial/final) & $0.50 / 0.10$ & Output Layer Nodes: SWRE & 1 \\
\hline Elite Selection & Above Avg & Output Layer Nodes: OARE & 9 \\
\hline Normalization Factor $(\lambda)$ & $50 / 20 / 10 / 5$ & Hands/Match (Evaluation) & 1000 \\
\hline Monte Carlo Sampling Size & $100 / 100 / \mathrm{NA}$ & Hands in Preliminary Session & 10000 \\
\hline
\end{tabular}

Table 7-1: ASHE 2.2 Parameters. Monte Carlo sampling is only applied to the first two betting rounds. The normalization factor values are listed by the betting round. Evolution is extended due to more parameters in the LSTM modules.

Recursive Utility Estimation cannot be done without reliable node stats from the PRTs. Hence, at the beginning of the preliminary session, the agents are essentially using the ASHE 2.0 decision algorithm. As more hands are played, recursive utility estimation is applied more often, and the agent's strategy may change noticeably as the preliminary session proceeds. To ensure that the node stats in the default PRT reflects the final strategy of the agent, the preliminary session for each generation contained twice as many hands as those in the ASHE 2.1 experiments.

Other than the above settings, the experimental setup (e.g. opponents for training and evaluation, match format, etc.) is the same as in the ASHE 2.1 experiments.

\subsubsection{Results and Analysis}

ASHE 2.2 was evolved by playing against two groups of training opponents. HM, SL, CM, and CS were used for the first 300 generations. LP, LA, TP, and TA were used in the remaining 450 generations. Default PRTs were constructed through self-playing in the preliminary sessions. 


\begin{tabular}{lllllll}
\hline & HM & CM & SL & CS & RG & HP \\
\hline AS 2.2 & $43105 \pm 6122$ & $45920 \pm 5139$ & $999 \pm 1.5$ & $12131 \pm 1730$ & $9204 \pm 759$ & $531 \pm 68$ \\
\hline AS 2.1 & $39998 \pm 5361$ & $44295 \pm 4994$ & $999 \pm 1.2$ & $10523 \pm 1594$ & $8598 \pm 703$ & $381 \pm 60$ \\
\hline AS 2.0 & $42333 \pm 6037$ & $46114 \pm 4985$ & $999 \pm 1.4$ & $9116 \pm 1403$ & $8996 \pm 721$ & $278 \pm 59$ \\
\hline
\end{tabular}

\begin{tabular}{lllllll}
\hline & LA & LP & TP & TA & SB & AS 2.0 \\
\hline AS 2.2 & $21304 \pm 1104$ & $16117 \pm 425$ & $1705 \pm 126$ & $604 \pm 91$ & $128 \pm 67$ & $139 \pm 59$ \\
\hline AS 2.1 & $19021 \pm 976$ & $15524 \pm 302$ & $1525 \pm 98$ & $535 \pm 76$ & $69 \pm 50$ & $62 \pm 43$ \\
\hline AS 2.0 & $20005 \pm 1006$ & $15372 \pm 634$ & $1488 \pm 91$ & $509 \pm 88$ & $5 \pm 61$ & - \\
\hline
\end{tabular}

Table 7-2: ASHE 2.2 Evaluation Results. Recursive Utility Estimation based on OARE and advanced PRTs improves ASHE's performance against low-exploitability opponents significantly. ASHE 2.2 defeated SB and ASHE 2.0 by over $1 / 10$ of a Big Blind per hand.

Table 7-2 shows the results. All performances are measured in $\mathrm{mBB} / \mathrm{hand}$. The performance of ASHE 2.1 (self-playing version) and ASHE 2.0 are listed for comparison. Evaluation is organized in the same way as the previous experiments, i.e. ASHE 2.2 played at least 20,000 duplicated hands against each opponent. An opponent's results are in blue if used during training.

Overall, Recursive Utility Estimation enhances ASHE's performance across the board. ASHE 2.2 is more effective against both low-exploitability and high-exploitability opponents than the previous versions. The performance gain is most significant against low-exploitability opponents. In particular, average winnings of ASHE 2.2 against Slumbot 2017 and ASHE 2.0 almost doubled compared to ASHE 2.1.

Game log analysis reveals that ASHE 2.2 applied advanced tactics that involve actions in multiple betting rounds much more effectively than the previous systems did, which is the main source of performance gain facing strong opponents. For instance, ASHE 2.2 exploited Slumbot 2017's excessive semi-bluff with flush draws through floating. The sample below is the 730th hand in a 1000-hand match during evaluation, which illustrates this tactic. 
ASHE 2.2 Slumbot 2017

\begin{tabular}{|c|c|c|c|}
\hline Private Cards & \multicolumn{2}{|c|}{ 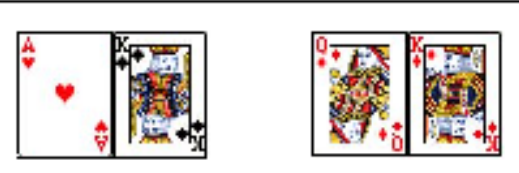 } & - \\
\hline Preflop & & & Pot \\
\hline Action \# & & & 150 \\
\hline 1 & Bet 300 & & 400 \\
\hline 2 & & Raise 900 & 1200 \\
\hline 3 & Call & & 1800 \\
\hline Flop & $4 \bullet \bullet 1^{8} \div$ & & \\
\hline 4 & & Check & 1800 \\
\hline 5 & Bet 900 & & 2700 \\
\hline 6 & & Raise 2700 & 5400 \\
\hline 7 & Call & & 7200 \\
\hline Turn & 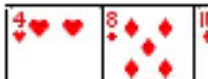 & $4+\infty$ & \\
\hline 8 & & Check & 7200 \\
\hline 9 & Bet 3600 & & 10800 \\
\hline 10 & & Fold & \\
\hline
\end{tabular}

Figure 7-3: Sample Game-ASHE 2.2 v. Slumbot 2017. ASHE exploited the opponent by floating, i.e. calling with a weak hand with the intention to bluff in later round(s).

In this hand, ASHE plays the Button and Slumbot the Big Blind. Slumbot 3-bet preflop with King-Queen of diamonds and ASHE called with Ace-King off-suit. The flop missed ASHE's hand completely, giving it nothing but Ace-high. ASHE continuation bet, and Slumbot responded with a semi-bluff raise. Recognizing the flush draw potential of the board using advanced PRTs, ASHE estimated the utility of each available action. Through Recursive Utility Estimation, the agent found that calling is the most profitable 
action given the current game state, because the opponent is likely to be holding a flush draw and fold it to a turn bet if the turn card does not complete the flush. Thus, ASHE called on the flop, and when the turn card missed the draw, it bluffed with a half-pot bet, winning the "bluff war" and a big pot.

In contrast, when facing similar situations, ASHE 2.0 tends to fold and ASHE 2.1 tends to re-raise (pot-size/all-in) on the flop. Both options are inferior: folding makes the opponent's semi-bluff profitable; re-raising on the flop offers the opponent better odds to call with two more cards to come and risks much more chips. Note that in order to make the optimal decision in such situations, future moves and community cards must be taken into consideration when evaluating action utilities. Hence, Recursive Utility Estimation and advanced PRTs is necessary for achieving high performance against low-exploitability opponents.

In sum, ASHE 2.2 outperformed the previous versions consistently and achieved considerably better results in matches against low-exploitability opponents. Experimental results show that Recursive Utility Estimation allows the agent to apply advanced poker tactics more effectively, which is the main source of the performance gain. 


\section{Chapter 8: Hand Range Analysis}

The ASHE 2.0 architecture contains four components: the PRTs, the two LSTM estimators, i.e. OFRE and SWRE, and the decision algorithm. ASHE 2.1 introduces the advanced PRTs, ASHE 2.2 replaces OFRE with OARE and employs Recursive Utility Estimation in the decision algorithm. This chapter focuses on improving SWRE. The idea is to enhance the accuracy of showdown value estimation by integrating the Hand-Range Estimation into ASHE 2.2. Experimental results show that such a system is most effective in modeling and exploiting both high-exploitability and low-exploitability opponents.

\subsection{HaNd RANGe ANALYSIS}

Hand range analysis is one of the most important tools for professional human players to decide their moves in poker. It means to evaluate the showdown equity of a hand based on the range of hands the opponent may have.

The probability of the opponent holding certain hand(s) can be inferred from the action sequence and the community cards. For example, suppose the hero holds AsAc, and the river board is Kh9h7s|2s|Ah. The hero has top set/three-of-a-kind, a reasonably strong hand. The villain checks the river, the hero bets half the pot, and the villain raises by the size of the pot. At this point, the hero is facing a tough decision.

From a hand range analysis perspective, rather than guessing what the villain is holding at the moment, the hero should ask: what is the range of hands with which the villain might raise? The hero should take the board, the actions in this hand, and the overall strategy of the opponent into consideration. Given this board, the villain could be holding a flush, which crushes hero's hand. However, flushes may not be the only hands in the villain's range. Here, action sequence can provide valuable clues. For instance, suppose the villain played aggressively preflop, he is likely to have Ace-King, Kings, nines, or even 
sevens, all of which are worse hands. The opponent's overall strategy is another important factor. If the villain is a maniac, it is entirely possible that he is simply bluffing on a "scary river" with a much worse hand. In contrast, if he plays tight and only raises with strong hands, the hero's hand may not be good enough against the villain's range to justify a call.

Through hand range analysis, good human players estimates showdown value of their hands more accurately, which usually leads to profitable decisions in the long run. The Hand Range Estimator in ASEH 2.3 is designed to do the same.

\subsection{HANd RANGe EStimator AND THE ASHE 2.3 ARCHiteCtURE}

Hand Range Estimator is introduced in ASHE 2.3 to improve showdown equity evaluation, which is critical for estimating action utility. In Recursive Utility Estimation, showdown equity, i.e. the probability of winning in a showdown, is used only at terminal states where all five community cards are dealt out or sampled by Monte Carlo method (Figure 7-1, line 3-4). In these states, seven out of the 52 cards are ruled out by either the board or the private cards, leaving the opponent with 990 possible combinations of private cards. While it is possible to predict opponent hand range based on their type (e.g. onepair, two-pair, three-of-a-kind, etc.) like most human players do, it is unnecessary since what matters is the probability of the opponent holding each of these combinations.

An important trade-off in the design of the Hand Range Estimator is between the specificity of hands and the complexity of the model. Predicting the probability of each combination may lead to more accurate estimation of showdown equity. Nevertheless, it requires a big network, resulting in slow training.

In ASHE 2.3, the HRE estimates the probabilities of the opponent's hand falling into one of the nine buckets. These buckets are not defined by the type of hands (e.g. a flush, straight, etc.). Instead, they correspond to different intervals in the ranking among 
the 990 possible hands. These intervals are: $1-25,26-50,51-100,101-150,151-$ $250,251-350,351-500,501-650$, and $651-990$. The intervals are defined narrower for higher ranks because high-ranking hands end up in a showdown more often and need to be distinguished from each other.

To estimate showdown equity using HRE predictions, ASHE's hand is compared with the possible hands in the opponent's range. Suppose a $r_{\mathrm{A}}$ is the number of hands that are stronger than ASHE's hand, showdown equity $p_{\text {win }}$ is computed as:

$$
\begin{gathered}
p_{\text {win }}=1-\sum_{i=1}^{N} p_{i} \delta\left(r_{\mathrm{A}}, a_{i}, b_{i}\right) \\
\delta\left(r_{\mathrm{A}}, a, b\right)=\left\{\begin{aligned}
0 & \text { if } r_{\mathrm{A}} \leq a \\
\frac{r_{\mathrm{A}}-a}{b-a} & \text { if } a<r_{\mathrm{A}} \leq b \\
1 & \text { if } b<r_{\mathrm{A}}
\end{aligned}\right.
\end{gathered}
$$

where $N$ is the number of intervals $(N=9), \mathrm{a}_{i}$ and $\mathrm{b}_{i}$ are the boundaries of interval $i$, and $p_{i}$ is the HRE prediction for interval $i$.

Similar to the OARE in ASHE 2.2, the HRE consists of a single layer of LSTM blocks and a fully-connected feed-forward estimation network with one hidden layer and nine output nodes. Softmax function is applied to normalize the outputs. Input features for the HRE are the same as those for the SWRE. As the Recursive Utility Estimation algorithm visits a node, the states of the HRE are saved. The inputs are then sent to the HRE. The LSTM states are restored before the algorithm returns from the PRT node.

Figure 8-1 presents the architecture of ASHE 2.3. Note that SWRE is replaced by the HRE to provide showdown equity estimation based on hand range. The ASHE 2.3 architecture is the most advanced version of the system in this dissertation and integrates all upgraded components introduced in the previous chapters. 


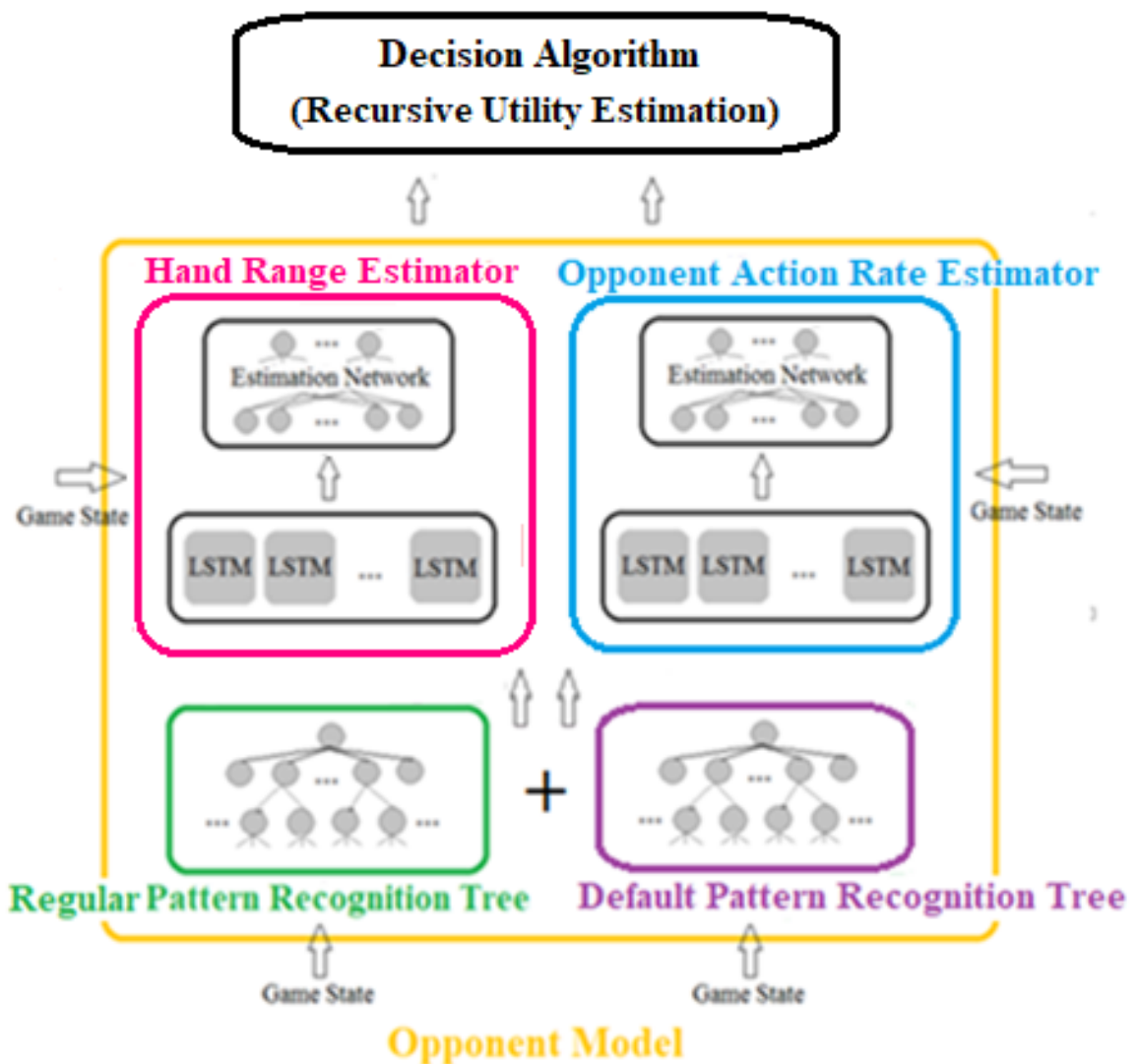

Figure 8-1: ASHE 2.3 Architecture. SWRE is replaced by the HRE for more accurate showdown value estimation. This system integrates all upgrades in Chapter 6 to 8, and performs the best in terms of exploiting flaws in opponent strategies and playing against strong opponents.

\subsection{EXPERIMENTAL RESULTS}

This section presents the experimental results on ASHE 2.3. The performance of all versions of the system against various opponents are compared and analyzed. The agents are also evaluated in a tournament. 


\subsubsection{Evolving ASHE 2.3}

Table 8-1 shows key parameters in the experiments. Values different from the ASHE 2.2 experiments are in blue. Evolution and modular session (i.e. consecutive generations dedicated for evolving each module) were extended due to larger number of parameters. Population size was also increased by $60 \%$.

In the first session where the HREs were frozen and OAREs evolved, the agents were allowed to cheat by using the actual winning probability (either 1 or 0 ) in the utility estimation. This approach helps the populations discover advantageous genotypes more efficiently. After the first session, the agents started using their HREs to predict showdown equity.

Other than the above, experimental setup was the same as before. ASHE 2.3 were evolved by playing against the high-exploitability group, i.e. HM, SL, CM, and CS, in the first 400 generations. The (relatively) low-exploitability group, i.e. LA, LP, TP, and TA, were used as the training opponents in the other 800 generations.

\begin{tabular}{llll}
\hline Parameter & Value & Parameter & Value \\
\hline Number of Generation & 1200 & LSTM Block Size & 10 \\
\hline Generation(s) per Session & 100 & Blocks per Module & 50 \\
\hline Population Size & 80 & Estimation Net Input Size & 500 \\
\hline Survival Rate & 0.30 & Estimation Net Hidden Layer & 1 \\
\hline Mutation Rate (initial/final) & $0.25 / 0.05$ & Estimation Net Hidden Nodes & 50 \\
\hline Mutation Strength (initial/final) & $0.50 / 0.10$ & Output Layer Nodes: HRE & 9 \\
\hline Elite Selection & Above Avg & Output Layer Nodes: OARE & 9 \\
\hline Normalization Factor $(\lambda)$ & $50 / 20 / 10 / 5$ & Hands/Match (Evaluation) & 1000 \\
\hline Monte Carlo Sampling Size & $100 / 100 / \mathrm{NA}$ & Hands in Preliminary Session & 10000 \\
\hline
\end{tabular}

Table 8-1: ASHE 2.3 Parameters. Evolution was extended to optimize the HREs, which contained more parameters than the SWRE.

\subsubsection{Performance vs. Highly Exploitable Opponents}

Table 8-2 compares the performance of all versions of ASHE against opponents that are highly exploitable. ASHE 1.0 were retrained using the same training opponents, 
i.e. the two groups, as the rest of the agents. The best results against each opponent are marked in bold. The superscript indicates key features/upgrades in each version of ASHE ( $\mathrm{R}=$ Recursive Utility Estimation, $\mathrm{H}=$ Hand Range Estimation, $\mathrm{P}=\mathrm{PRT}, \mathrm{P}+=$ advanced PRT, $\mathrm{i}=$ implicit modeling, and e = explicit modeling).

Through opponent modeling and adaptation, the ASHE series were able to exploit weak opponents $40 \%$ to $1700 \%$ more effectively than Slumbot 2017 . These results show that low exploitability does not equal to high utility in imperfect information games such as poker. Opponent modeling and adaptation can lead to huge performance advantage in these games.

\begin{tabular}{|c|c|c|c|c|}
\hline & HM & $\mathrm{CM}$ & SL & $\mathrm{CS}$ \\
\hline ASHE $2.3^{\mathrm{eP}+\mathrm{RH}}$ & $46025 \pm 6694$ & $48790 \pm 5512$ & $999 \pm 1.4$ & $14267 \pm 1798$ \\
\hline ASHE $2.2^{\mathrm{eP}+\mathrm{R}}$ & $43105 \pm 6122$ & $45920 \pm 5139$ & $999 \pm 1.5$ & $12131 \pm 1730$ \\
\hline ASHE $2.1^{\mathrm{eP}+}$ & $39998 \pm 5361$ & $44295 \pm 4994$ & $999 \pm 1.2$ & $10523 \pm 1594$ \\
\hline ASHE $2.0^{\mathrm{eP}}$ & $42333 \pm 6037$ & $46114 \pm 4985$ & $999 \pm 1.4$ & $9116 \pm 1403$ \\
\hline ASHE $1.0^{\mathrm{i}}$ & $39228 \pm 5022$ & $42776 \pm 4150$ & $998 \pm 1.8$ & $9462 \pm 1666$ \\
\hline Slumbot 2017 & $4988 \pm 881$ & $2761 \pm 355$ & $702 \pm 59$ & $4512 \pm 472$ \\
\hline & LA & LP & $\mathrm{TP}$ & TA \\
\hline ASHE $2.3^{\mathrm{eP}+\mathrm{RH}}$ & $24608 \pm 1765$ & $17015 \pm 721$ & $1744 \pm 153$ & $626 \pm 102$ \\
\hline ASHE $2.2^{\mathrm{eP}+\mathrm{R}}$ & $21304 \pm 1104$ & $16117 \pm 425$ & $1705 \pm 126$ & $604 \pm 91$ \\
\hline ASHE $2.1^{\mathrm{eP}+}$ & $19021 \pm 976$ & $15524 \pm 302$ & $1525 \pm 98$ & $535 \pm 76$ \\
\hline ASHE $2.0^{\mathrm{eP}}$ & $20005 \pm 1006$ & $15372 \pm 634$ & $1488 \pm 91$ & $509 \pm 88$ \\
\hline ASHE $1.0^{i}$ & $16541 \pm 890$ & $10241 \pm 391$ & $982 \pm 102$ & $134 \pm 59$ \\
\hline Slumbot 2017 & $2449 \pm 460$ & $623 \pm 41$ & $603 \pm 51$ & $284 \pm 49$ \\
\hline
\end{tabular}

Table 8-2: ASHE Systems v. Highly Exploitable Opponents. The series are significantly more effective in exploiting weak opponents than Slumbot 2017, the best equilibriumbased agent that is made publicly available. ASHE 2.3, which integrates advanced PRT, Recursive Utility Estimation, and Hand Range Estimator outperformed the other versions in matches against most opponents.

Within the ASHE Series, each version brings in new techniques that improve the performance. ASHE 2.3, the most advanced version in this dissertation, integrates Hand Rand Estimation, Recursive Utility Estimation, and advanced PRTs into the ASHE 2.0 
explicit opponent modeling framework, and achieved the best performances against highexploitability opponents among all variants of the system.

In particular, HRE improves ASHE's showdown equity estimation when facing opponents with ultra-high exploitability (e.g. HM and CM.) by predicting the probability of the lowest two ranges to be roughly the same as the other seven ranges. This prediction captures the important fact that actions from these agents are essentially irrelevant to the value of their hand. This prediction allowed ASHE 2.3 to outperform the 2.2 version in matches against these opponents by noticeable margin.

The HRE also played a critical role in matches against CS, whose aggression level honestly reflects the value of its hand. Game logs revealed that the HRE achieved 78.5\% overall accuracy in predicting the opponent's hand value ${ }^{1}$. The average accuracy reached $90 \%$ in the last 500 hands in each 1000-hand match.

\subsubsection{Tournament Evaluation}

To evaluate ASHE's performance facing opponents that are not seen during training, a tournament was held among RG, HP, SB and all versions of ASHE. In this tournament, each pair of agents played at least 20,000 duplicated hands against each other, organized into 1000-hand matches as usual. If the winner cannot be decided with statistical significance, more hands were played up to 100,000 for each pair of agents. Table 8-3 presents the results of the tournament.

The agents are ranked (as indicated in the superscripts) first by their wins/losses and then by the total earnings in the tournament. ASHE 2.3 is the champion, and ASHE 2.2 the runner-up. All four ASHE 2.x agents rank higher than Slumbot 2017.

\footnotetext{
${ }^{1}$ A prediction is correct if the opponent's hand falls into the hand range with the maximum probability according to the HRE.
} 


\begin{tabular}{|c|c|c|c|c|c|c|c|c|}
\hline & AS 2.3 & AS 2.2 & AS 2.1 & AS 2.0 & SB & HP & AS 1.0 & RG \\
\hline AS $2.3^{1 \mathrm{st}}$ & & 9.5 & 109 & 132 & 141 & 549 & 1094 & 11227 \\
\hline $\operatorname{AS~} 2.2^{2 \mathrm{nd}}$ & -9.5 & ( & 98 & 139 & 128 & 531 & 1150 & 9204 \\
\hline AS $2.1^{3 r d}$ & -109 & -98 & & 62 & 69 & 381 & 638 & 8598 \\
\hline AS $2.0^{4 \text { th }}$ & -132 & -139 & -62 & & 11.4 & 278 & 581 & 8996 \\
\hline $\mathrm{SB}^{5 \text { th }}$ & -141 & -128 & -69 & -11.4 & & 152 & 269 & 2102 \\
\hline $\mathrm{HP}^{7 \mathrm{th}}$ & -549 & -531 & -381 & -278 & -152 & 102 & 89 & 1413 \\
\hline AS $1.0^{6 \text { th }}$ & -1094 & -1150 & -638 & -581 & -269 & -89 & & 7984 \\
\hline $\mathrm{RG}^{8 \mathrm{th}}$ & -11227 & -9204 & -8598 & -8996 & -2102 & -1413 & -7984 & \\
\hline
\end{tabular}

Table 8-3: Tournament Evaluation Results. The colors red, yellow, and green indicate the agent in the row losing to, tying with, or winning the agent in the column. The ASHE Systems are effective against both static and dynamic opponents that are not seen during evolution. ASHE 2.1 and later versions of the system defeated Slumbot 2017. ASHE 2.3 and ASHE 2.2 performed the best in matches against low-exploitability opponents.

ASHE 2.3 and 2.2 were both undefeated in the tournament. The two agents tied statistically after 1000 matches (100,000 duplicated hands) against each other. The HRE gave ASHE 2.3 an edge in total earnings by beating RG with a bigger margin. Therefore, the championship was awarded to ASHE 2.3.

Empirical results indicate that the HRE is more effective in predicting showdown values against weaker opponents. The performance advantage of ASHE 2.3 over ASHE 2.2 tends to be smaller when facing low-exploitability opponents. In heads-up matches between these two agents, overall the accuracy of the HRE predictions was $32.7 \%$, which increased to around $35 \%$ in the last 500 hands. Note that the accuracy was significantly lower than the accuracy when facing CS. This difference is likely to be caused by adaptation, which makes hand range prediction more difficult.

Overall, ASHE 2.3 and 2.2 demonstrate strong generalizability to opponents that are not seen during training. In particular, both agents exploited ASHE 1.0 effectively by check-raising and/or slow playing their strong hands. They were also able to discover and exploit a big "leak" in HP's strategy: the agent's calling range was too tight when facing 
raises on the river. Furthermore, the performance of ASHE 2.3 and 2.2 in matches against SB, HP, RG and the previous versions in the ASHE Series show that the system can model and exploit both dynamic and static strategies effectively.

Thus, the ASHE Series provide a new approach to building adaptive agents for HUNL and other imperfect information games. The next chapter summarizes this line of research and discusses potential directions for future work. 


\section{Chapter 9: Discussion and Future Work}

This chapter discusses ASHE's limitations and suggests potential directions for future work in two aspects: (1) training and evaluation, (2) reinforcement learning, and (3) end-to-end learning and generalization to other problem domains.

\subsection{Training ANd Evaluation}

The ASHE agents are mainly evolved by playing against opponents that are highly or moderately exploitable. Empirical results demonstrate the remarkable generalizability of this approach. A set of reasonably diversified training opponents is usually sufficient for evolving agents that can generalize well to new opponents with much lower exploitability. Nevertheless, experimental results also indicate that training opponents with lower exploitability tend to yield better performance than highly exploitable opponents with the same types of weakness.

In addition, opponents can be organized into training groups according to their level of exploitability. During evolution, training groups with higher exploitability can be used first, followed by groups with lower exploitability. This method promotes adaptation and exploitation more effectively, which leads to higher performance. Hence, it would be interesting to see how performance can be improved if more low-exploitability agents are used during training and evaluation.

While the plan is straightforward, it can be difficult to access high-quality HUNL agents. Most state-of-the-art poker agents such as DeepStack and Libratus are not publicly available due to the huge computational resource requirement. The Annual Computer Poker Competition (ACPC) used to be the best way to access high-quality agents. Submissions were made accessible to participants by convention. A prototype of ASHE 2.0 was submitted to the 2017 ACPC and ranked the seventh among fifteen submissions in 
overall performance (http://www.computerpokercompetition.org). However, due to budget issues and technical difficulties, the 2017 results were incomplete, and the submissions were not made available. In addition, the 2018 ACPC results are not released at the time of writing, and the submissions are also not accessible.

For the above reason, the best available low-exploitability opponent, i.e. Slumbot, was used in the experiments in this dissertation. Slumbot 2016 (used in Chapter 4) was the runner-up of for the $2016 \mathrm{ACPC}$, and Slumbot 2017 (used in Chapter 5 to 8) is the latest version of the agent. A potential direction for future work is to evolve and evaluate the system with stronger opponents when they become available.

\subsection{REINFORCEMENT LEARNING}

Neuroevolution has been shown to be more effective than standard reinforcement learning techniques in many benchmark tasks with sparse reinforcement such as helicopter control [Koppejan and Whiteson, 2009] and pole balancing [Moriarty and Miikkulainen 1996; Heidrich-Meisner and Igel, 2009]. In particular, compact and effective neural network structures can be discovered efficiently through genetic algorithms such as NEAT [Stanley and Miikkulainen, 2002]. Thus, this dissertation employs evolutionary methods for training the RNN-based opponent models in ASHE.

However, it is also possible to apply reinforcement learning techniques to building computer agents for poker and other imperfect information games. Heinrich and Silver [2016] combined fictitious self-play with deep reinforcement learning to approximate Nash equilibrium strategies in Leduc poker and Limit Holdem, whose performance approached the state of the art. Nevertheless, the agents were not adaptive. Hence, a possible direction for future work is to apply deep RL to opponent modeling and exploitation in HUNL. For 
instance, earnings/losses in each hand can be used as rewards to adjust the agent's actions during lifetime, thus allowing the agents to adapt to different opponent strategies.

\subsection{Generalization}

In principle, the architectural framework of the ASHE Series and the evolutionary method to train the system are applicable to other domains with imperfect information, if they are modeled as a formal game. Thus, another direction for future work is to generalize the proposed method in other problem domains.

A natural extension of the system is to develop an agent for multi-player tournament poker. The main challenge is to represent the enormous game state space efficiently. One possible approach is to train additional neural network modules to identify the "strongest opponent", thus converting the multi-player game into a heads-up game. Another option is to train a neural network encoder to compress the game states. Agents can be trained to operate on the compressed/abstracted game instead, which is similar to abstraction in Nash equilibrium approximation.

In addition, ASHE can be applied to games other than poker. For instance, in a security game between the attacker and the defender, PRTs can be constructed to represent the action sequences. LSTM modules can be evolved to predict the form and target of the attacks. Recursive Utility Estimation can be used to estimate expected utility for each defensive measure. However, as in the case of poker, feature engineering is necessary in adjusting the system. In particular, the PRT node stats and the input features for the LSTM modules are defined based on domain knowledge, which is problem-specific.

While most existing methods in building computer agents for imperfect information games (e.g. CFR and other equilibrium approximation techniques) rely heavily on domain knowledge, such reliance makes generalization more difficult. Therefore, another potential 
direction for future work is to improve generalizability by reducing the dependency of domain-specific knowledge and human interference in feature extraction. The goal is to develop an end-to-end RNN-based adaptive poker agents with high performance. Yakovenko, et al [2016] proposed creating a poker playing system using a unified poker representation. A convolutional-neural-network-based model was developed to learn the patterns in three variants of poker, including Limit Texas Holdem. This approach may be combined with the ASHE 2.x architecture to reduce expert knowledge needed in feature extraction. 


\section{Chapter 10: Contributions and Conclusion}

This chapter summarizes the work from Chapter 4 to 8 . Section 10.1 outlines the contributions of this work. Section 10.2 evaluates its impact.

\subsection{Contributions}

From ASHE 1.0 to ASHE 2.3, the ASHE Series provide an effective new approach to building high-performance adaptive agents for HUNL and other imperfect information games. ASHE 1.0 uses LSTM modules, i.e. the opponent module and the game module, to extract and encode opponent strategy and game states from the current hand. These features are then sent to a feed-forward neural network, i.e. the decision network, which calculates an aggression score. Actions are chosen by a rule-based decision algorithm according to the score. To address the problem of insufficient training data for supervised learning, the system is evolved through neuroevolution. Tiered Survival and Elite Reproduction (TSER) is introduced to resolve the dilemma between offspring quality and genotype preservation in a stochastic game. Fitness is evaluated by Average Normalized Earnings (ANEs) against highly exploitable opponents that require different counterstrategies for exploitation.

Empirical results show that ASHE 1.0 is far more effective than Slumbot 2016, one of the top-ranking Nash-equilibrium-based agents, in matches against highly exploitable opponents. This result demonstrates ASHE's ability to model and exploit the opponents. In addition, ASHE 1.0 evolved through playing against highly exploitable opponents can generalize to stronger opponents that are not seen in training. However, the agent is defeated by Slumbot in heads-up matches with relatively big margins.

ASHE 1.0 validates the design principle of RNN-based adaptive agents in largescale imperfect information games and lays a solid foundation for discovering opponent models through evolutionary methods. The main shortcoming is that opponent modeling 
in ASHE 1.0 is implicit. The agent does not predict opponent actions or evaluate showdown value. Everything is encoded by the aggression score, which leads to less accurate decisionmaking.

ASHE 2.0 solves this problem with an extended architecture that models the opponents explicitly. LSTM neural networks are employed to estimate the showdown value and predict the opponent's probability of folding given bets/raises of different size. The decision algorithm evaluates the expected utilities of available actions using these predictions and decides the agent's move accordingly. A new data structure, i.e. Pattern Recognition Tree (PRT), is introduced to maintain statistics on opponent strategy given different game states. The PRTs benefit the system in two ways: (1) they provide useful features for the LSTM estimators, and (2) they reduce the size of the LSTM modules, thus improving training efficiency.

Experimental results show that ASHE 2.0 inherits the ability to model and exploit weak opponents from ASHE 1.0. In addition, the agent ties statistically with Slumbot 2017, which is an upgrade and much stronger version of the equilibrium-based agent used in the ASHE 1.0 experiments. Moreover, the agent can model and exploit both static and dynamic opponents effectively. ASHE 2.0 establishes an architectural framework for LSTM-based adaptive agents with explicit opponent modeling. Key components in the architectural framework, i.e. the PRTs, the LSTM estimators, and the decision algorithm are then upgraded in ASHE 2.1 to 2.3, each focusing on a different aspect.

ASHE 2.1 introduces the advanced PRTs, which contain default PRTs and boardtexture-based PRTs. The former allows the agent to make better decisions facing new opponents or infrequent game states, and the latter enables the agent to extract subtle patterns in opponent strategy that are based on the texture of the board. The advanced PRTs 
are particularly effective against low-exploitability opponents; ASHE 2.1 defeats both ASHE 2.0 and Slumbot 2017 by statistically significant margins.

ASHE 2.2 introduces Recursive Utility Estimation to evaluate action utility more accurately. This technique allows the agent to take future moves into consideration when choosing its actions. The LSTM estimators are extended to predict probability of different actions from the opponent. Experimental results show that Recursive Utility Estimation allows ASHE to apply advanced poker tactics more effectively, thus enhancing performance against both high and low exploitability opponents.

ASHE 2.3 introduces Hand Range Estimator (HRE) to improve showdown value estimation. The HRE predicts the probability of the opponent's hand falling into different hand strength interval. Showdown values are then calculated based on statistical rules. Empirical results indicate that the HRE enhances overall performance of the agent and is especially effective in predicting the hand range of high-exploitability opponents. Tournament evaluation show that ASHE 2.3 has the best overall performance among the ASHE series, tying heads-up against ASHE 2.2 and defeating all other versions as well as Slumbot 2017.

Thus, the ASHE series of poker agents provide an effective new approach to building RNN-based adaptive agents for HUNL through evolutionary method. The architectural framework and genetic algorithm should be generalizable to other imperfect information games.

\subsection{CONCLUSION}

This dissertation presents the first evolutionary method for building RNN-based adaptive agents that model and exploit their opponents in Heads-Up No-Limit Holdem. A series of poker agents called Adaptive System for Holdem (ASHE) were built to evaluate 
the proposed approach. Experimental results show that compared to Nash-equilibriumbased agents, the ASHE Series are generally far more effective against flawed strategies due to opponent modeling and adaptation. Moreover, given reasonably diversified training opponents, ASHE 2.x are able to model and exploit both static and dynamic opponent strategies with much lower exploitability. In particular, ASHE 2.1 and later versions of the system were able to defeat top-ranking equilibrium-based agents in heads-up matches.

Thus, this dissertation provides an effective new approach for building adaptive agents for poker and other imperfect information games and points out a promising new direction for research on such games. In particular, opponent modeling and exploitation using evolved recurrent neural networks may be the next step beyond Nash equilibrium approximation for building computer agents for imperfect information games. In the future, the ASHE architecture and evolutionary method in the dissertation can applied to other decision-making problems with imperfect information. 


\section{References}

Bard, N., Johanson, M., Burch, N., and Bowling, M. (2013). Online Implicit Agent Modeling. In Proceedings of the 12th International Conference on Autonomous Agents and Multi-agent Systems (AAMAS 2013), Saint Paul, Minnesota, USA.

Billings, D., Papps, D., Schaeffer, J., and Szafron, D. (1998). Opponent Modeling in Poker. In Proceedings of the Joint Conference of AAAI/IAAI, 1998.

Billings, D., Davidson, A., Schaeffer. J, and Szafron, D. (2002). The challenge of poker. Artificial Intelligence 134, 201-240.

Billings, D., Burch, N., Davidson, A., Holte, R., Schaeffer, J., Schauenberg, T., Szafron, D. (2003). Approximating Game-Theoretic Optimal Strategies for Full-scale Poker. In The Eighteenth International Joint Conference on Artificial Intelligence.

Billings, D. (2006). Algorithms and Assessment in Computer Poker. Ph.D. Dissertation, University of Alberta.

Bowling, M., Burch, N., Johanson, M., and Tammelin, O. (2015). Heads-up limit holdem poker is solved. Science, 347(6218):145-149.

Brown, N., and Sandholm, T. (2014). Regret transfer and parameter optimization. In Proceedings of the AAAI Conference on Artificial Intelligence (AAAI).

Brown, N. and Sandholm, T. (2017). Safe and Nested Endgame Solving for ImperfectInformation Games. In the AAAI workshop on Computer Poker and Imperfect Information Games.

Brown, N., Ganzfried, S., and Sand-holm, T. (2015). Hierarchical Abstraction, Distributed Equilibrium Computation, and Post-Processing, with Application to a Champion No-Limit Texas Hold'em Agent. In Proceedings of the International Conference on Autonomous Agents and Multi-agent Systems.

Burch, N., Johanson, M., and Bowling, M. (2014). Solving Imperfect Information Games Using Decomposition. In AAAI Conference on Artificial Intelligence (AAAI).

Cai, G., Wurman, P. (2005). Monte Carlo Approximation in Incomplete Information, Sequential Auction Games, Decision Support Systems, v.39 n.2, p.153-168.

Davidson, A., Billings, D., Schaeffer, J., and Szafron, D. (2000). Improved Opponent Modeling in Poker. In International Conference on Artificial Intelligence, ICAI'00.

Doetsch, P., Kozielski, M., and Ney, H. (2014). Fast and Robust Training of Recurrent Neural Networks for Offline Handwriting Recognition. In the 14th International Conference on Frontiers in Handwriting Recognition. 
Ekmekci, O., and Sirin, V. (2013). Learning Strategies for Opponent Modeling in Poker. In Proceedings of Workshops at the Twenty-Seventh AAAI Conference on Artificial Intelligence. Bellevue: AAAI Press (pp. 6-12).

Fan, Y., Qian, Y., Xie, F., and Soong, F. (2014). TTS Synthesis with Bidirectional LSTMbased Recurrent neural networks. In Proc. Interspeech.

Fitzgerald, A. (2015). Range Analysis: The Correct Way to Analyze Poker Hands. In Jonathan Little's Excelling at No-Limit Hold'em, D\&B Publishing.

Ganzfried, S. (2016). Bayesian Opponent Exploitation in Imperfect-Information Games. In AAAI Workshop on Computer Poker and Incomplete Information.

Ganzfried, S. and Sandholm, T. (2011). Game Theory-Based Opponent Modeling in Large Imperfect-Information Games. In Proceedings of the International Conference on Autonomous Agents and Multi-Agent Systems.

Ganzfried, S. and Sandholm, T. (2014). Potential-aware Imperfect-Recall Abstraction with Earth-Mover's Distance in Imperfect Information Games. In AAAI Workshop on Computer Poker and Imperfect Information Games.

Ganzfried, S. and Sandholm, T. (2015a). Endgame Solving in Large Imperfect Information Games. In International Conference on Autonomous Agents and Multi-Agent Systems (AAMAS).

Ganzfried, S. and Sandholm, T. (2015b). Safe Opponent Exploitation. In ACM Transactions on Economics and Computation, 3(2), 8.

Gilpin, A.; Hoda, S.; Pena, J.; and Sandholm, T. (2007a). Gradient-based Algorithms for Finding Nash Equilibria in Extensive Form Games. In International Workshop on Internet and Network Economics (WINE).

Gilpin, A., Sandholm, T., and Sorensen, T. (2007b). Potential-aware Automated Abstraction of Sequential Games. In Proceedings of the 22nd national conference on Artificial intelligence, pp.50-57, Vancouver, British Columbia, Canada.

Gilpin, A. and Sandholm, T. (2005). Optimal Rhode Island Hold'em poker, in Proceedings of the 20th national conference on Artificial intelligence, p.1684-1685, Pittsburgh, Pennsylvania.

Gilpin, A. and Sandholm, T. (2006a). A Competitive Texas Holdem Poker Player via Automated Abstraction and Real-time Equilibrium Computation. In Proceedings of the National Conf. on Artificial Intelligence (AAAI).

Gilpin, A., and Sandholm, T. (2006b). Finding Equilibria in Large Extensive Form Games of Imperfect Information. In Proceedings of the 7th ACM conference on Electronic commerce. 
Gilpin, A. and Sandholm, T. (2008a). Solving Two-Person Zero-Sum Repeated Games of Incomplete Information. In Proceedings of the Seventh International Conference on Autonomous Agents and Multi-Agent Systems.

Gilpin, A., and Sandholm, T. (2008b). Expectation-based Versus Potential-aware Automated Abstraction in Imperfect Information Games: An Experimental Comparison Using Poker. In Proceedings of theAAAI Conference on Artificial Intelligence (AAAI).

Gomez, F. and Miikkulainen, R (1999). Solving Non-markovian Control Tasks with Neuroevolution. In Proceedings of the Sixteenth International Joint Conference on Artificial Intelligence, pp. 1356-1361, San Francisco, California, USA.

Graves, A., and Schmidhuber, J. (2005). Framewise Phoneme Classification with Bidirectional LSTM and Other Neural Network Architectures. Neural Networks, 18(5-6):602-610.

Graves, A., Liwicki, M., Bunke, H., Schmidhuber, J., and Fernandez, S. (2008). Unconstrained Online Handwriting Recognition with Recurrent Neural Networks. In Advances in Neural Information Processing Systems, pp. 577-584.

Greff, K., Srivastava, R. K., Jan, K., Steunebrink, B. R., and Schmidhuber, J. (2015). LSTM: a Search Space Odyssey. arXiv preprint arXiv:1503.04069.

Gruau, F., Whitley, D., and Pyeatt, L. (1996). A Comparison between Cellular Encoding and Direct Encoding for Genetic Neural Networks. In Proceedings of the First Annual Conference, pp. 81-89, MIT Press, Cambridge, Massachusetts.

Heidrich-Meisner, V. and Igel, C. (2009). Neuroevolution Strategies for Episodic Reinforcement Learning, Journal of Algorithms, Vol 64, pp 152-168.

Heinrich, J. and Silver, D. (2016). Deep Reinforcement Learning from Self-play in Imperfect Information Games. In NIPS 2016 Deep Reinforcement Learning Workshop.

Hochreiter, S. and Schmidhuber, J. (1997). Long Short Term Memory. Neural Computation 9, pp 1735-1780.

Hoda, S., Gilpin, A., and Pena, J. (2006). A Gradient-based Approach for Computing Nash Equilibria of Large Sequential Games. In INFORMS.

Jackson, E. (2016). Compact CFR. In AAAI Work-shop on Computer Poker and Imperfect Information Games, Phoenix, AZ, USA.

Jackson, E. (2017). Targeted CFR. In AAAI Work-shop on Computer Poker and Imperfect Information Games, San Francisco, CA, USA.

Johanson, M. and Bowling, M. (2009). Data Biased Robust Counter Strategies, In Proceedings of the Twelfth International Conference on Artificial Intelligence and Statistics (AISTATS). 
Johanson, M. (2013). Measuring the Size of Large No-Limit Poker Games. arXiv:1302.7008 [cs.GT].

Johanson, M., Burch, N., Valenzano, R., and Bowling, M. (2013). Evaluating State-space Abstractions in Extensive Form Games. In Proceedings of the International Conference on Autonomous Agents and Multi-Agent Systems (AAMAS).

Johanson, M., Zinkevich, M., and Bowling, M. (2008). Computing Robust CounterStrategies. In Advances in Neural Information Processing Systems 20 (NIPS).

Koller, D., Megiddo, N., and von Stengel, B. (1996). Efficient Computation of Equilibria for Extensive Two-person Games. In Games and Economic Behavior 14(2):247259.

Koller, D. and Pfeffer, A. (1997). Representations and Solutions for Game-theoretic Problems. In Artificial Intelligence, pages 167-215.

Koppejan, R. and Whiteson, S. (2009) Neuroevolutionary Reinforcement Learning for Generalized Helicopter Control, In Proceedings of the $11^{\text {th }}$ Annual Conference on Genetic and Evolutionary Computation (GECCO 09), pp 145-152.

Korb, K., Nicholson, A., and Jitnah, N. (1999). Bayesian Poker. In Proceedings of the Fifteenth Conference on Uncertainty in Artificial Intelligence, pp. 343-350. Morgan Kaufmann Publishers Inc.

Koutnik, J., Schmidhuber, J., and Gomez, F. (2014). Evolving Deep Unsupervised Convolutional Networks for Vision-based Reinforcement Learning. In Proceedings of the 2014 Conference on Genetic and Evolutionary Computation (GECCO 2014), pp. 541-548.

Kuhn, H. (1950). A Simplified Two-person Poker. Contributions to the Theory of Games, 1:97-103.

Lanctot, M., Waugh, K., Zinkevich, M., and Bowling, M. (2009). Monte Carlo Sampling for Regret Minimization in Extensive Games. In Advances in Neural Information Processing Systems (NIPS), pp.1078-1086.

Lehman, J. and Miikkulainen, R. (2014). Overcoming Deception in Evolution of Cognitive Behaviors. In Proceedings of the Genetic and Evolutionary Computation Conference (GECCO), Vancouver, BC, Canada, July.

Li, X. and Miikkulainen, R. (2014). Evolving Multimodal Behavior through Subtask and Switch Neural Networks. In Proceedings of the Fourteenth International Conference on the Synthesis and Simulation of Living Systems.

Li, X. and Miikkulainen, R. (2017). Evolving Adaptive Poker Players for Effective Opponent Exploitation. In AAAI Workshop on Computer Poker and Imperfect Information Games, San Francisco, CA. 
Li, X. and Miikkulainen, R (2018) Opponent Modeling and Exploitation in Poker Using Evolved Recurrent Neural Networks. In Proceedings of the 2018 Conference on Genetic and Evolutionary Computation (GECCO 2018).

Lisy, V. and Bowling, M. (2016). Equilibrium Approximation Quality of Current No-Limit Poker Bots, arXiv preprint arXiv:1612.07547.

Lockett, A., and Miikkulainen, R. (2008). Evolving Opponent Models for Texas Hold'em. In Proceedings of the IEEE Conference on Computational Intelligence in Games, IEEE Press.

Luong, T., Sutskever, I., Le, Quoc V., Vinyals, O. and Zaremba, W. (2014). Addressing the Rare Word Problem in Neural Machine Translation. arXiv:1410.8206.

Marchi, E., Ferroni, G., Eyben, F., Gabrielli, L., Squartini, S., and Schuller, B. (2014). Multiresolution Linear Prediction-based Features for Audio Onset Detection with Bidirectional LSTM Neural Networks. In 2014 IEEE International Conference on Acoustics, Speech and Signal Processing (ICASSP), pp. 2164-2168.

Miltersen, P. B., and Sørensen, T. B. (2006). Computing Sequential Equilibria for TwoPlayer games. In Annual ACMSIAM Symposium on Discrete Algorithms (SODA), pp. 107-116.

Moravcik, M., Schmid, M., Burch, N., Lisy, V., Morrill, D., Bard, N., Davis, T., Waugh, K., Johanson, M., and Bowling, M. (2017). DeepStack: Expert-Level Artificial Intelligence in No-Limit Poker, axXiv:1701. 01724.

Moriarty, D. E. and Miikkulainen, R. (1996). Efficient Reinforcement Learning through Symbiotic Evolution. Machine Learning, v.22 n. 1-3, pp 11-32.

Moriarty, D. E. and Miikkulainen, R. (1997). Forming Neural Networks through Efficient and Adaptive Co-evolution. In Evolutionary Computation, 5(4):373-399.

Nash, J. (1951). Non-cooperative Games. In the Annals of Mathematics, Second Series, Vol 54, Issue 2 (Sep., 1951), pp. 286-295.

Norris, K. and Watson, I. (2013). A Statistical Exploitation Module for Texas Hold'em and Its Benefits When Used With an Approximate Nash Equilibrium Strategy. In Proceedings of IEEE Conference on Computational Intelligence and Games, Niagara, Canada.

Ponsen, M., Ramon, J., Croonenborghs, T., Driessens, K., and Tuyls, K. (2008). Bayes Relational Learning of Opponent Models from Incomplete Information in No-Limit Poker. In Twenty-third Conference of the Association for the Advancement of Artificial Intelligence (AAAI-08).

Ponsen, M., Jong, S., and Lanctot, M. (2011). Computing Approximate Nash Equilibria and Robust Best Responses Using Sampling. In Journal of Artificial Intelligence Research. 
Potter, M. A., De Jong, K. A., and Grefenstette, J. J. (1995). A Coevolutionary Approach to Learning Sequential Decision Rules. In Proceedings of the Sixth International Conference on Genetic Algorithms, pp. 366-372, Morgan Kaufmann, San Francisco, California, USA.

Rawal, A. and Miikkulainen, R. (2016). Evolving Deep LSTMs Networks Using Information Maximization Objective. In Proceedings of the Genetic and Evolutionary Computation Conference, Denver, CO.

Sajjan, S., Sankardas, R., Dipankar, D. (2014). Game Theory for Cyber Security. In Proceedings of the Sixth Annual Workshop on Cyber Security and Information Intelligence Research, April 21-23, 2010, Oak Ridge, Tennessee, USA

Sak, H., Senior, A., and Beaufays, F. (2014). Long Short-Term Memory Recurrent Neural Network Architectures for Large Scale Acoustic Modeling. In Proceedings of the Annual Conference of International Speech Communication Association (INTERSPEECH).

Sakaguchi, M. and Saka, S. (1992). Solutions of Some Three-person Stud and Draw Poker. Mathematics Japonica, pages 1147-1160.

Schrum, J. and Miikkulainen, R. (2014). Evolving Multimodal Behavior With Modular Neural Networks in Ms. Pac-Man. In Proceedings of the Genetic and Evolutionary Computation Conference (GECCO).

Selby, A (1999). Optimal Heads-up Preflop Poker. www.archduke.demon.co.uk/simplex.

Shi, J. and Littman, M. (2001). Abstraction Models for Game Theoretic Poker. In Computers and Games, pp. 333-345. Springer-Verlag.

Southey, F., Bowling, M., Larson, B., Piccione, C., Burch, N., Billings, D., and Rayner, C (2005). Bayes Bluff: Opponent Modelling in Poker. In Proceedings of the 21st Annual Conference on Uncertainty in Artificial Intelligence.

Stanley, K. and Miikkulainen, R. (2002). Evolving Neural Networks through Augmenting Topologies. In Evolutionary Computation. 10 (2): 99-127.

Stanley, K., Bryant, B., and Miikkulainen, R. (2005). Real-time Neuroevolution in the NERO Video Game. In IEEE Transactions on Evolutionary Computation, Vol. 9 , No. 6.

Takusagawa., K (2000). Nash Equilibrium of Texas Hold'em Poker. Undergraduate thesis, Computer Science, Stanford University.

Tammelin, O.; Burch, N., Johanson, M., and Bowling, M. (2015). Solving heads-up limit Texas Holdem. In Proceedings of the 24th International Joint Conference on Artificial Intelligence (IJCAI). 
Teofilo, L. F., Reis, L. P. (2011). Identifying Player's Strategies in No Limit Texas Hold'em Poker through the Analysis of Individual Moves, In Proceedings of the 15th Portuguese Conference on Artificial Intelligence. Lisbon, Portugal.

Vatsa, S., Sural, S., Majumdar, A.K. (2005). A Game-theoretic Approach to Credit Card Fraud Detection. In Proceedings of the First Int'l Conf. Information Systems Security, pp. 263-276.

Von Neumann, J., (1928). Zur Theorie der Gesellschaftsspiele Math. Annalen. 100 (1928) pp. 295-320.

Wang, X. and Wellman, M. (2017). Spoofing the Limit Order Book: An Agent-based Model. In AAAI Workshop on Computer Poker and Imperfect Information Games, San Francisco, CA.

Waugh, K.; Zinkevich, M.; Johanson, M.; Kan, M.; Schnizlein, D.; and Bowling, M. (2009). A Practical Use of Imperfect Recall. In Proceedings of the Symposium on Abstraction, Reformulation and Approximation (SARA).

Whitley, D., Dominic, S, Das, R., and Anderson, C. (1993). Genetic Reinforcement Learning for Neurocontrol problems. In Machine Learning, 13:259-284.

Yakovenko, N., Cao, L., Raffel, C., and Fan, J. (2016). Poker-CNN: A Pattern Learning Strategy for Making Draws and Bets in Poker Games Using Convolutional Neural Networks. In Proceedings of the $30^{\text {th }}$ AAAI Conference on Artificial Intelligence.

Zaremba, W., Sutskever, I., and Vinyals, O. (2014). Recurrent Neural Network Regularization. arXiv:1409.2329 [cs].

Zinkevich, M., Johanson, M., Bowling, M., and Piccione, C. (2007). Regret Minimization in Games with Incomplete Information. In Proceedings of the Twenty-First Annual Conference on Neural Information Processing Systems (NIPS). 